\title{
Sending all the right signals
}

Citation for published version (APA):

Francu, R. E. (2019). Sending all the right signals: How social signaling drives creativity in B2B new service development. [Doctoral Thesis, Maastricht University]. Off Page Amsterdam. https://doi.org/10.26481/dis.20190606rf

Document status and date:

Published: 01/01/2019

DOI:

10.26481/dis.20190606rf

Document Version:

Publisher's PDF, also known as Version of record

\section{Please check the document version of this publication:}

- A submitted manuscript is the version of the article upon submission and before peer-review. There can be important differences between the submitted version and the official published version of record.

People interested in the research are advised to contact the author for the final version of the publication, or visit the DOI to the publisher's website.

- The final author version and the galley proof are versions of the publication after peer review.

- The final published version features the final layout of the paper including the volume, issue and page numbers.

Link to publication

\footnotetext{
General rights rights.

- You may freely distribute the URL identifying the publication in the public portal. please follow below link for the End User Agreement:

www.umlib.nl/taverne-license

Take down policy

If you believe that this document breaches copyright please contact us at:

repository@maastrichtuniversity.nl

providing details and we will investigate your claim.
}

Copyright and moral rights for the publications made accessible in the public portal are retained by the authors and/or other copyright owners and it is a condition of accessing publications that users recognise and abide by the legal requirements associated with these

- Users may download and print one copy of any publication from the public portal for the purpose of private study or research.

- You may not further distribute the material or use it for any profit-making activity or commercial gain

If the publication is distributed under the terms of Article $25 \mathrm{fa}$ of the Dutch Copyright Act, indicated by the "Taverne" license above, 


\title{
SENDING ALL THE RIGHT SIGNALS
}

How Social Signaling Drives Creativity in B2B New Service Development

\author{
By \\ RUXANDRA - ELENA FRANCU
}


SENDING ALL THE RIGHT SIGNALS. How Social Signaling Drives Creativity in B2B New Service Development

(c) Copyright Ruxandra - Elena Francu, Maastricht, The Netherlands, 2019

Cover design, $\mathrm{LEGO}^{\circ}$ Lightbulb model, and dissertation layout: Ruxandra - Elena Francu Printing: Off Page, Amsterdam, The Netherlands

ISBN: 978-94-6182-960-3

All rights reserved. No part of this publication may be reprinted or utilized in any form or by any electronic, mechanical, or other means, now known, or hereafter invented, including photocopying and recording, or in any information storage or retrieval system, without permission from the copyright owner. 
SENDING ALL THE RIGHT SIGNALS

How Social Signaling Drives Creativity in B2B New Service

Development

\section{DISSERTATION}

to obtain the degree of Doctor at Maastricht University,

on the authority of the Rector Magnificus, Prof. Dr. Rianne M. Letschert, in accordance with the decision of the Board of Deans, to be defended in public

on Thursday, June $6^{\text {th }}, 2019$, at 12:00 hours

By

RUXANDRA - ELENA FRANCU 


\section{Supervisors:}

Prof. Dr. Jos G.A.M. Lemmink

Prof. Dr. Mien S.R. Segers

\section{Co-supervisor:}

Dr. Piet G.C. van den Bossche

\section{Assessment Committee ${ }^{1}$ :}

Prof. Dr. Martin G.M. Wetzels - Committee Chair

Prof. Dr. Wim H. Gijselaers

Prof. Dr. Annouk Lievens, University of Antwerp, Antwerp, Belgium

Prof. Dr. Alex 'Sandy' Pentland, Massachusetts Institute of Technology (MIT), Cambridge, USA

\footnotetext{
${ }^{1}$ The members of the Assessment Committee are listed in alphabetical order, after the Chair
} 
To my mother and my husband 


\section{Acknowledgements}


This book symbolizes the merger of two realms that had inhabited me since I can remember: 'sociology and untangling social interaction' and 'motherboards and data'. Upon reflection, the road leading up to this moment was not at all smooth, but I cherished every second of it, as the dream of conducting my doctoral research with the Sociometers was becoming real one pixel at the time, and the research curiosity was building up unquenchably as the data, the results, the findings were pouring in. The following chapters describe the findings of my research into how the way we come together influences the outcomes of our interactions. At a meta-level, you are now reading a piece of work that is in and of itself the outcome of interactions I had with people who crossed my Ph.D. path and that shaped it one way or the other. I would like to express my gratitude for their wisdom, support, and lessons taught.

Prof. Dr. Jos Lemmink, dearest Jos, thank you for selecting me to conduct the MBA casestudy back in 2012, for the amazingly insightful conversations we had while traveling for the project that allowed me to pitch what would eventually become my doctoral research. Thank you for the carte-blanche you gave me at the start, for believing in me, for ceaselessly supporting me along the way.

Prof. Dr. Mien Segers, dear Mien, thank you for joining Jos in guiding my Ph.D. trajectory, for believing in the potential of my ideas, for your masterful theoretical guidance that helped refine them, for the healthy dose of skepticism you carefully raised during our methodological research meetings, for your critical and constructive feedback that helped me to refine my studies in their pivotal moments.

Dr. Piet van den Bossche, dear Piet, thank you for accepting to become my daily supervisor, thank you for your methodical support, for making the time to meet with me every Friday, for being so closely involved in helping me refine the design of my studies, for your invaluable feedback to all my writings, be it proposals, conference papers, presentation slides or otherwise.

Jos, Mien, and Piet thank you for the gentle diplomacy with which you handled my stubbornness. Thank you for being there for me. Thank you for your patience. Thank you for being my role models. Thank you for everything. I am deeply beholden to you.

I would like to thank Océ Technologies B.V. for partially funding my Ph.D. position.

I am extremely indebted to the companies that so graciously agreed to collaborate on the empirical studies presented in this dissertation. Thank you very much for your interest, time, collaboration, and instrumental support.

Next, I would like to express my deep gratitude to the members of my Degree Committee.

Prof. Dr. Martin Wetzels, thank you for accepting to chair the assessment of my manuscript. I am deeply thankful for your guiding the completion of my Ph.D. research journey as I equally am for your initiating it, by hiring me as a Ph.D. candidate and by enabling the purchase of the Sociometers. Thank you ever so much for believing in the potential of my research.

Prof. Dr. Wim Gijselaers, thank you for accepting to join the committee to assess my Ph.D. manuscript. Thank you also for welcoming me into ERD's vibrant research environment from even before the actual start of my Ph.D. and for allowing me to join the stimulating "ERD team research" team. 
Prof. Dr. Annouk Lievens, thank you for accepting to join the committee to assess my Ph.D. manuscript. Thank you also for being an academic role model in the broad sense as well as in the services marketing research realm.

Prof. Dr. Alex 'Sandy' Pentland, thank you for accepting to join the committee to assess my Ph.D. manuscript. Thank you also for imparting your research philosophy that fundamentally changed who I am as a researcher. Thank you for your groundbreaking work that materialized in the creation of the Sociometer and its successors. Thank you for your invaluable feedback and for your thought leadership.

Prof. Dr. Martin Wetzels, Prof. Dr. Wim Gijselaers, Prof. Dr. Annouk Lievens, Prof. Dr. Alex 'Sandy' Pentland, I am honored and deeply humbled to have your names alongside those of my supervisors engraved on my Ph.D. manuscript and guiding my future academic path.

I would like to extend my gratitude to the members of my Corona for your challenging questions in support of my projects.

I would also like to thank my co-author, Dr. Lucas, dear Ben, thank you for joining my second research project and becoming my "research co-conspirator". Not only were you the first to believe in my wild research ideas besides my supervisors, but you proved to me they were not wild enough. To say this dissertation would not have materialized in this form without your input, skills, research enthusiasm, and matching-mad work ethic is a flagrant understatement. You made the last part of my Ph.D. journey terrifyingly challenging, thought provoking, but all so joyful and rewarding. Thank you for all the machine-learning trainings, for all the countless hours spent at the office helping me automate the curation of my thousands-something matrices. Thank you for being my work's restless PR agent, for nagging me into believing in my work. Thank you for supporting our Cambridge and Dartmouth collaboration and for the research visits to "the place that gave the world computer science". In the process, you became the best friend I could have ever asked for, my sturdy support, and the beam of confidence in my professional abilities I so desperately needed. I look forward to continuing our collaboration and to reaching our goal of publishing our joint work in the National Academy of Sciences... someday.

Prior to starting my Ph.D. I was fortunate enough to join the Service Science Factory (SSF) team where my interest in service design was truly ignited and where I learnt and applied its methodologies. Prof. Dr. Gaby Odekerken - Schröder, dear Gaby, thank you for your guidance and leadership during my time at SSF and thereafter. Linda, Vanessa, Laszlo, Maartje, and Conny thank you for your support and friendship during my SSF journey.

I would like to also extend my appreciation to my colleagues at the Department of Marketing and Supply Chain Management who have been there along the way.

First and foremost, Eefije and Pascalle, thank you for being my research and emotional support system throughout my Ph.D.

Secondly I would like to thank my senior colleagues Gaby, Caroline, Dominik, Lisa, Bram, Kelly, Lieven, Niels, Diogo, Anouk and my senior and fellow Ph.D. candidates Anne, Francisco and Stefania, Hannes, Jan (Pelser), Jan (Meyer), Johannes, Katja, Liz, Nina, Robert, Stefan, Theo, Wiebke for providing a thought-provoking research environment and for your collegiality. Alex, thank you for being one of the linchpins of my career by recommending me for Jos's MBA case-study project. I will be forever grateful for this and for your support and friendship while our paths crossed. 
Thirdly, thank you to the dear "baby-Ph.Ds.", Letícia, Hannah, Kimberley, Piet, Pieter, Steffi and last, but definitely not least, Susan for the fun chats during those much-needed coffee breaks and for indulging me some parsimonious knowledge-parting; I hope it helped.

Most importantly, thank you to the lovely office-mates I had over the years Vera, Nadine, Tim and Anja, Anika, Kars, Ruud, and Teerawut for putting up with my fidgeting around with my devices, for your kind support and advice, for the fun times we spent together, and for making our office a delightful place to come to and work in.

I would also like to thank my SBE colleagues Alexandra, Aline, Caren, Christine, Catherine, Dinah and Lars, Dominik F., Evangelia, Ewa, Florian, Joey, Katerina, Lennart, Maike, Mieke, Mukul, Selma, Sonja, Sofya, and Therese for the great time spent chatting over coffees, bouncing ideas, trying to diffuse Ph.D.-related frustrations, and sharing our little victories along the way.

Thank you to all of the above whom I had met as colleagues but became and remained dear friends I deeply appreciate.

I would like to express my gratitude to my co-authors from the Computer Laboratory at University of Cambridge, Prof. Dr. Cecilia Mascolo, Dr. Alessandro Montanari, and Brian Jones and from the Department of Computer Science at Dartmouth College, Prof. Dr. Xia Zhou and Zhao Tian. Our joint project and its resulting paper were an amazing experience that fueled my academic ambitions. I am deeply honored and thankful for having been part of your team and I very much look forward to our future collaborations.

During my Ph.D. I had the pleasure to supervise and teach some great students and, at times, I had the real pleasure of working with a handful of the brilliant ones. André Frenz, Lena Wiegmann, Pauline Koehler, Stefanie Hornig, Julius Dietzel, and Christopher Brooks thank you all for your amazing work and dedication; thank you for making my work feel ever so meaningful.

A giant "thank you" to Marleen and Judith for accepting to be my paranymphs and for committing to having my literal and proverbial back on this June $6^{\text {th }}$, on my "Defense -Day"! Marleen, the only constant in my Ph.D. journey (besides my supervisors), thank you for being my voice of reason, my trusted soundboard for matters of work and life, thank you for being (at the same time) The Doctor and an amazing companion for travelling into life's "big ball of wibbly wobbly, timey wimey stuff". Above all, thank you from the bottom of "both my hearts" for being such an amazingly brilliant, kind, and funny friend. Judith, the joyous and witty accounting Ph.D. swiftly turned reliable and dear friend, thank you for being there for me, for your razor-sharp advice, and for the fun times.

A warm "thank you" to you, Rita, for your friendship, for your eye-opening advice, for the lovely early morning pup-walks, and, of course, for teaching me Dutch.

Octave, thank you for being an amazing vocal coach, for our deep talks, for hanging out with your puppies, and for helping me find my voice, both literally and figuratively.

To my dearest and closest friends Corina and Rareș, Alina, Ana, Dana, Raluca-Patricia, my "Dulvacs", Ralu and Andrei.... Thank you for sticking with me through thick and thin over the years, for being there for me, for lifting me up, for giving me reasons to smile and carry on, for brightening up my life, for walking in when everyone else walked out, for everything. Thank you for being my beloved family of choice. 
Bunicilor mei dragi, Victor și Lidia, vă mulțumesc pentru susţinerea necontenită, pentru dragostea pe care mi-o purați și pentru tot ce m-ați învățat de-a lungul anilor. Sper că v-am făcut cât de puțin mândri, că dorul care ne-a separat a meritat îndurat și că rezultatul, cartea de față, e un semn că am dus ștafeta la o line de final care vă bucură.

Laur, îți mulțumesc că ai apărut și rămas în viețile noastre, că ne-ai ajutat să învățăm să devenim pentru prima dată o familie. Îți mulțumesc pentru dragostea și grija pe care o ai de mama zi de zi, în special în zilele când uită să aibă grijă de ea. Îți mulțumesc pentru toate sacrificiile pe care le-ai facut pentru noi și sper să mă pot revanșa pentru tot într-o bună zi.

To my adored pup, Hemi... For over 11 and a half years you have walked beside me as my deeply loving companion. Your pitter-patter, your cuddles, your kisses gave me a reason to hang in through thick and thicker. You helped me overcome everything life threw at me because, let's face it, irrespective of everything, someone had to walk you. And feed you. And love you to the moon and back. And raise you into the most adorable and majestic dog in the world. And provide the socks. And then love you even more. No matter what our lives will bring, I will be forever grateful for having had a glimpse into parenthood and experiencing how pure love feels like.

Grand-papa, these are the hardest words to write... as deeply as I miss you, you are here, with me, in spirit, every waking day... I like to think you watched your "gâgă" find her way back to motherboards and circuits.... I am always guided by your teachings; I am forever motivated by the passion and curiosity you had for technical innovation that you also imbued in me since I was a toddler when we were "innovating transistors" together in your lab. I hope this book makes you proud.

Mama... The love I have for you is ineffable. I don't know how I will ever thank you for everything you have done for me. You are and you have always been my mother, my father, my baby-sister, my older sister, my grandparent, my best friend, my confidant, my aunt \& uncle, my life teacher, my strategist... my family, my all! I would not have made it here had it not been for you. This book is a culmination of your relentless support and love as the single constant in my life. You are the kindest and most genuine soul crowned with a brilliant mind... The best in me I owe to you.

Ben, my husband, my best friend, my sidekick, my love, you have found a fast-track into my soul and, once there, you never left. And, luckily, I have this ring on my finger that says you never will. Just as you so relentlessly supported me professionally, you cherish and empower me, you help grow as a person, and you bring meaning to my life day in, day out. Thank you for your contagious zest for life, optimism, and grit. Thank you ever so deeply for all the love, joy, and laughter you bring into my life every single day. I can't wait to spend the rest of them with you!

R. Elena Francu, Nottingham, England April 30 ${ }^{\text {th }}, 2019$ 


\section{Table of Contents}

Acknowledgements

Chapter 1 | Introduction

2

The Human Side of Innovation __ 4

The LEGO Metaphor__ 4

Overall Problem Statement ___ 5

1. Unraveling New Product Development, New Service Development, and Service Innovation

2. Customer Involvement in NPD/NSD _ 7

2.1. Types of Customer Involvement___ 8

2.2. Involvement of Consumers $\left(\mathrm{B}_{2} \mathrm{C}\right)$ and Customers $\left(\mathrm{B}_{2} \mathrm{~B}\right) \_9$

3. Creative Problem Solving___ 9

The Objectives of the Dissertation 10

The Structure of the Dissertation __ 11

Summary of Chapter 2: Capturing the Right Signals: Method Addendum ___ 11

Summary of Chapter 3: It Takes Two to Tango __ 11

Summary of Chapter 4: For Your Ties Only ___ 11

Theoretical Lens _ 13

Chapter 2 | Capturing the Right Signals: Method Addendum __ 16

1. Background _ 18

2. Social Signals - "New" Types of Data ___ 18

3. Social Signal Processing - Capturing and Dealing with New Types of Data ___ 19

4. Machine Learning Algorithms - "New" Tools for Analyzing "New" Data___ 20

5. Self-Reports as Ground-Truth for Classification _ 21

6. “Abduction": Data-Driven Theory Construction___ 22

7. Capturing the Right Signals: Conducting Studies with Sociometric Badges ___ 23

7.1. Stage A: Preparation for Data Collection___ 24

7.2. Stage B: Mechanics of the Day of the Deployment__ 24

7.3. Stage C: Data Management and Preparation for Analysis __ 25

8. Deploying Sociometric Badges "In the Wild" __ 26

Chapter 3 | It Takes Two to Tango 28

1. Introduction 30

2. Literature review__ 32

2.1. Customer Involvement, Creativity, and Innovation___ 32

2.2. Nonverbal Behavior and Creativity __ 33

2.2.1. Effects of Nonverbal Communication__ 34

2.2.2. Antecedents of Creativity___ 38

2.3. Research Questions and Research Aim ___ 38

3. Method 40

3.1. Participants and Setting __ 40

3.2. Meetings' Format ___ 40 
3.3. Procedure 42

3.4. Data Collection and Variables 43

3.5. Data Manipulation and Variables' Operationalization __ 44

3.5.1. Time Spent in Proximity [PRX] _ 45

3.5.2. Time Spent Face-To-Face $\left[\mathrm{F}_{2} \mathrm{~F}\right] \_45$

3.5.3. Turn-taking [TTK]___ 45

3.5.4. Relative Time Spent in Positive Body Movement (Or Energy) Mirroring $[\mathrm{BMM}]$ and in Positive Posture Mirroring [PM] _ 45

3.5.5. Creativity _ 46

4. Analysis _ 46

5. Results _ 47

5.1. Descriptives for Global Network Measures___ 47

5.2. Multiple Regressions with Quadratic Assignment Procedure (MRQAP)__ 49

5.3. Multi-class Classification __ 51

5.3.1. Forward Feature Selection (FFS)

5.3.2. Multi-class Classification with a Random Forest Classifier

5.4. Results__ 54

5.4.1. Forward Feature Selection (FFS) 54

5.4.2. Multi-class Classification with a Random Forest Classifier

6. Discussion _ـ 57

6.1. Methodological Contributions 58

6.2. Theoretical Contributions__ 59

6.3. Practical Implications _ 60

6.4. Limitations __ 60

6.5. Future Research Directions__ 61

Appendix 1. Firmware configuration 62

Appendix 2. Example of an unfolded multiplex matrix with three nodes and three layers and the respective graphs ___ 63

Appendix 3. Descriptive statistics__ 64

Appendix 4. Example of K-fold $(\mathrm{K}=10)$ validation for a subset of 7 dyads___ 66

Chapter 4 | For Your Ties Only__ 68

1. Introduction _ـ 69

2. Literature Review _ 71

3. Research Questions and Research Aim __ 73

4. Method 74

4.1. Participants and Setting __ 74

4.2. Meeting Format _ 74

4.3. Data Collection Procedure ___ 74

4.4. Variables' Operationalization and Plan for Analysis _ 75

4.5. Methodological Objectives ___ 78

4.6. Many-to-Many Pairwise Network Alignment___ 78

4.7. Creative Activation __ 79

5. Results 79 
5.1. Descriptives 79

5.1.1. Alignments 'By Meeting'____ 79

5.1.2. Alignments 'By Variable'___ 81

5.2. Network Alignment Evaluation __ 82

6. Discussion __ 85

6.1. Methodological Contributions__ 85

6.2. Theoretical Contributions___ 86

6.3. Practical Implications __ 87

6.4. Limitations__ 87

6.5. Future Research Directions___ 87

Appendix 1. The split of sessions across project meetings across teams ___ 89

Appendix 2. Stabilization coefficient versus mean for calculating the mirroring ties _ 90

Appendix 3. — 92

A. Example of alignment

B. Examples of High-Low Same-Other creative activation in two graphlets __ 92

Appendix 4. Overview of the input data for the classifier___ 93

Chapter 5 | Conclusion__ 96

1. Synopsis of Findings___ 97

2. Overall Implications__ 99

2.1. Chapter 2: Capturing the Right Signals: Method Addendum ___

2.2. Chapter 3: It Takes Two to Tango ___ 100

2.3. Chapter 4: For Your Ties Only___ 101

3. Concluding Remarks ___ 101

Chapter 6 | Valorization Addendum 104

1. The LEGO Light Bulb Metaphor__ 105

2. Social Signals__ 105

3. Implications for Practice __ 106

Bibliography____ 110

Summary _ 132

About the author___ 136

\section{List of Figures}

\section{Chapter 1 | Introduction}

Figure 1. Depiction of constituent literature streams .................................................................................

\section{Chapter 3 It Takes Two to Tango}

Figure 1. The "heart-beat" NSD project process ……......................................................................... 40

Figure 2. The ideation phase of the project process. ................................................................................42

Figure 3. Description of the adjacency matrix representing the number of turns recorded for each badge

in exchange with all other badges aggregated at a certain timeframe. ………………………..... 45

Figure 4. Topological overview of average densities per episode per variable. .........................................48

Figure 5. Meeting 1 - Topological overview of networks depicting the relative amount time spent in positive posture mirroring in the six composing layers: $[\mathrm{PM} 1]$ to $[\mathrm{PM} 6]$.................................50 
Figure 6. Meeting 2 - Topological overview of the networks depicting the amount time spent face-toface in the twelve composing layers: $\left[\mathrm{F}_{2} \mathrm{~F}_{1}\right]$ to $\left[\mathrm{F}_{2} \mathrm{~F}_{12}\right]$

Figure 7. Meeting 3 - Topological overview of the networks depicting the amount turns taken in the eleven composing layers: [TTK 1] to [TTK 11]....

Figure 8. Example of predicted probabilities in a binary classification

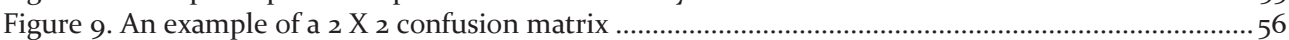

\section{Chapter 4 | For Your Ties Only}

Figure 1. An example multiplex network showing the nonverbal social interaction (NVSI) variables in one-time layer (i.e., 30 minutes)

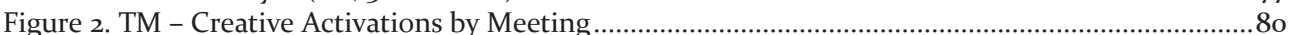

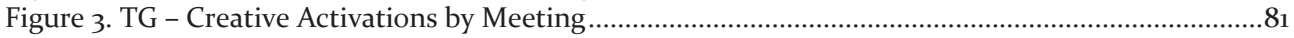

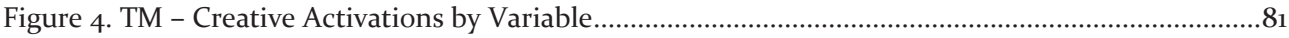

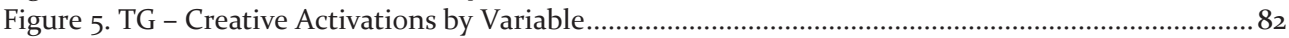

\section{List of Tables}

\section{Chapter 1 | Introduction}

Table 1. Overview of empirical studies.

\section{Chapter 3 It Takes Two to Tango}

Table 1. The detailed description of the episodes per meeting..................................................41

Table 2. Models: regression coefficients and model fit .......................................................... 50

Table 3. The first dyad in layer two of meeting 1 .................................................................52

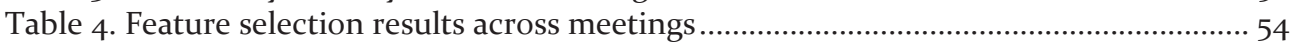

Table 5. Confusion matrices for each meeting................................................................... 56

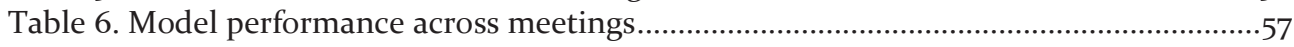

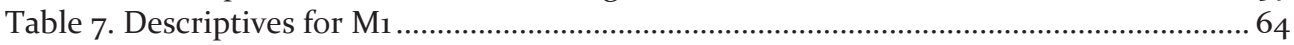

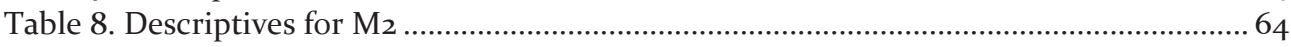

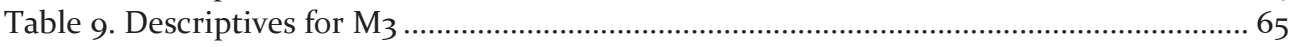

\section{Chapter 4 | For Your Ties Only}

Table 1. Network Alignment Evaluation Results for TM ..................................................... 83

Table 2. Network Alignment Evaluation Results for TG ..................................................... 83

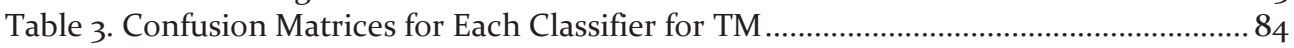

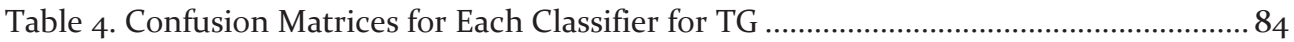

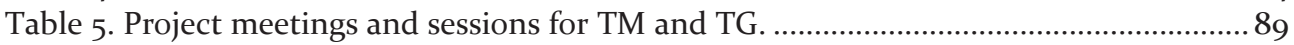

Table 6: Differences between the mean and the SC .............................................................. 91

Table 6. Raw counts for alignments 'by meeting' and 'by variable' for TM and TG .............. 93

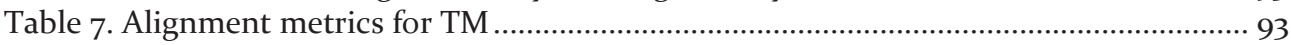

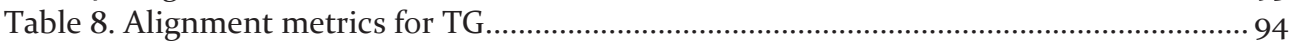

\section{Chapter 5 | Conclusion}

Table 1. Overview of results for Chapter 3 …….................................................................. 98

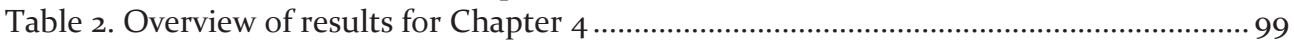




\section{Chapter 1}

\section{Introduction}




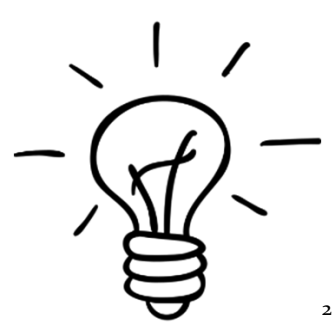

"The electric light did not come from the continuous improvement of candles."

- Oren Harari

The first lightbulb is a simple example of "accidental innovation", a piece of technology that eventually arose from Humphrey Davy's invention of the very first electric lamp with incandescent lighting, who built upon Alessandro Volta's discovery of continuous sources of electricity. Developed outside of the candle manufacturing industry, this technology revolutionized the way we illuminate our sorroundings slowly replacing the candle, even changing our circadian cycles as daylight was no longer a prerequisite for human activity. From the first building block set in 1800 by Volta to, for instance, Philip's new wellbeingfostering and cost-effective lighting solutions for smart-businesses and smart-cities, the chain of innovations lives on and is ever morphing.

This brief reflection upon this avant la lettre radical innovation and its aftermath highlights a few teachings. Firstly, as acknowledged many times before, radical innovation does not come about from continuously improving existing tools or technologies; it arises from developments in quasi-related fields. Secondly, as they spread, radical innovations change behaviors and create new markets. Thirdly, it is a contextually dependent process whereby the creation and success of an innovation is highly dependent on other supporting advancements in the same or adjecient fields.

The third teaching also highlights that innovation is obvious a posteriori; it is difficult to anticipate what the sequence of steps leading to innovation is and how each of these steps contributes to the final outcome. In hindsight, innovation should be 20/203; meaning that, in hindsight, innovation like any other complex journey, should be well documented for reflection such that the exact roads taken can be learnt from again in the future. Given though that innovation is arrived at via creativity, an inherently highly variable process, the question is, is there a reliable way to identify the markers and milestones of innovation as to predict it happening in actu?

\footnotetext{
${ }^{2}$ Icon made by Freepik from www.flaticon.com

3 Referring to the idiom "Hindsight is (always) 20/20". The idiom refers is to the acuity of perfect eyesight (i.e., $20 / 20$ vision) and it means that events, decisions, happenings, etc., are clearer post-facto, when reflected upon.
} 


\section{The Human Side of Innovation}

Since early research into communities-of-practice, interaction among people has been recognized as central to innovation (e.g., Brown \& Duguid, 1991). Along these lines, innovation in organizations has been viewed from the perspective of leading for creativity, and increasingly, from a perspective emphasizing collaboration. The latter includes recognition of the need for volume and diversity of ideas and the people generating them (Minor, Brook \& Bernoff, 2017; Rozovsky, 2015). Further, to add to the pool of ideas, and to ensure customer-centric ideation within innovation processes, many organizations now involve customers in such processes (Saldanha, Mithas \& Krishnan, 2017; Storey \& Larbig, 2018; Trischler, Pervan \& Kelly et al., 2018).

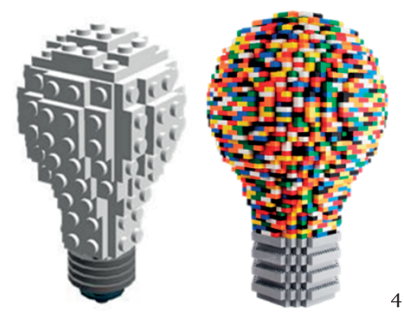

\section{The LEGO Metaphor}

Expressed in simple figurative terms, imagine a team of people tasked with developing a new product or service that we will metaphorically describe as a LEGO model of a lightbulb; the task is defined without exact specifications, and assuming the team afforded the decision of the model's final form (i.e., its shape, size, and color pattern). Now imagine that each team member's own ideas (stemming from prior knowledge, perspectives, experiences, etc.) are represented as LEGO bricks that come in different shapes and sizes but of only one color per team member (the bricks' colors are thus homogeneous within subjects, heterogeneous across subjects). From the onset, the team is in possession of a large number of LEGO bricks of different shapes, sizes, and colors that could lead to millions of possible combinations. However, even with a clear understanding of all available bricks, an observer would not likely be able to predict the final appearance of the model. For example, if we only consider six bricks of six times eight studs of one color there are 915,103,765 possible building combinations (Eilers, 2016). Therefore, the model's final form could be anything as the potential spatial and chromatic combinations grow exponentially with the number of team members and of ideas.

As the team members start interacting to build the model at every point in time during the building process the two mechanisms at play are (1) the individuals' ability to select the most appropriate bricks (i.e., the individual creative potential) and (2) the team's negotiation outcomes on the bricks' selection (i.e., deciding upon the suitable ideas) and on the placement of bricks (i.e., designing the implementation of ideas). In other words, the way

\footnotetext{
${ }^{4}$ Sources: left lightbulb - http://blog.mindcreations.com/archives/104o/lightbulb?lang=en; right lightbulb - http://invention.si.edu/build-lego-lightbulb
} 
they come together ultimately dictates (1) what individual ideas are shared and, respectively, (2) how the most valuable individual ideas are selected by the team and implemented. Extant theory recognizes the individuals' creative potential as a major source of creative input for innovation (Bharadwaj \& Menon, 2000; Scott \& Bruce, 1994) and acknowledges that individuals demonstrate different levels of creative potential (e.g., McCrae, 1987). In the context of the metaphor, this translates into each individual having a distinct amount of appropriate bricks (i.e., valuable ideas) and, the more suitable bricks an individual has, the greater the probability of their respective color to be exhibited on the final model. Additionally, it was confirmed by the psychometric literature that creative individuals have a unique ability for engaging in a stochastic combinatorial process (Simonton, 2003). This means that individuals are capable of foreseeing a certain distribution of bricks or their pattern (i.e., certain solutions) created with their own parts (i.e., own ideas).

Recent theoretical developments extoll the significance of the individuals' creative potential but emphasize that it is triggered in the (social) interaction between team members (PerrySmith \& Shalley, 2003). This stream of literature investigates how the interactions shape the individual creative process (Brennan \& Enns, 2015; De Jaegher, Di Paolo, \& Gallagher, 2010; Heerey, 2015) and lay the groundwork to understanding how they could influence the team creative process. In the context of the metaphor this means that the team interaction ultimately defines the characteristics of the model's final form (i.e., its shape, size, and color pattern).

\section{Overall Problem Statement}

Ideally, team literature should describe what kind of team interactions lead to certain types of creative outcomes. In reality, firstly, most team research has focused on team effectiveness and teams' performance outcomes (Salas, Reyes, \& McDaniel, 2018; Salas, Shuffler, Thayer, Bedwell, \& Lazzara, 2015). These studies mostly employed the input-process-output model assuming a quantifiable outcome (i.e., a performance measure); in contrast, an innovative outcome, is not and, more often than not, cannot be deliberately defined a priori as it emerges from its creative process. Secondly, the team dynamics studies conducted to date have been cross-sectional in nature (Mathieu, Hollenbeck, van Knippenberg, \& Ilgen, 2017; Mathieu, Tannenbaum, Donsbach, \& Alliger, 2014). Thirdly, an overwhelming majority of studies have investigated team behaviors by measuring them with self or other reports using retrospective surveys. Consequentially, a growing number of calls plea for collecting better data on teams (i.e., of objective nature, of higher granularity, longitudinally, etc.) (e.g., Kozlowski, 2015; Pentland, 2012).

This present dissertation proposes an emphasis on quantifying the objective behavior of teams (Shapiro, 2018) and aims to uncover how team creative mechanisms can be explained by objective and fine-grained behaviors in real-life new product or service development situations.

This thesis investigates the application of wearable technology (i.e., Sociometric badges) for the study of said objective human behavioral cues (or "social signals" such as vocalics, proxemics, and kinesics) to generate insights into the dynamics of new product and service 
development teams. Each of the literature streams drawn from informs crucial theoretical and methodological foundations of the composing studies.

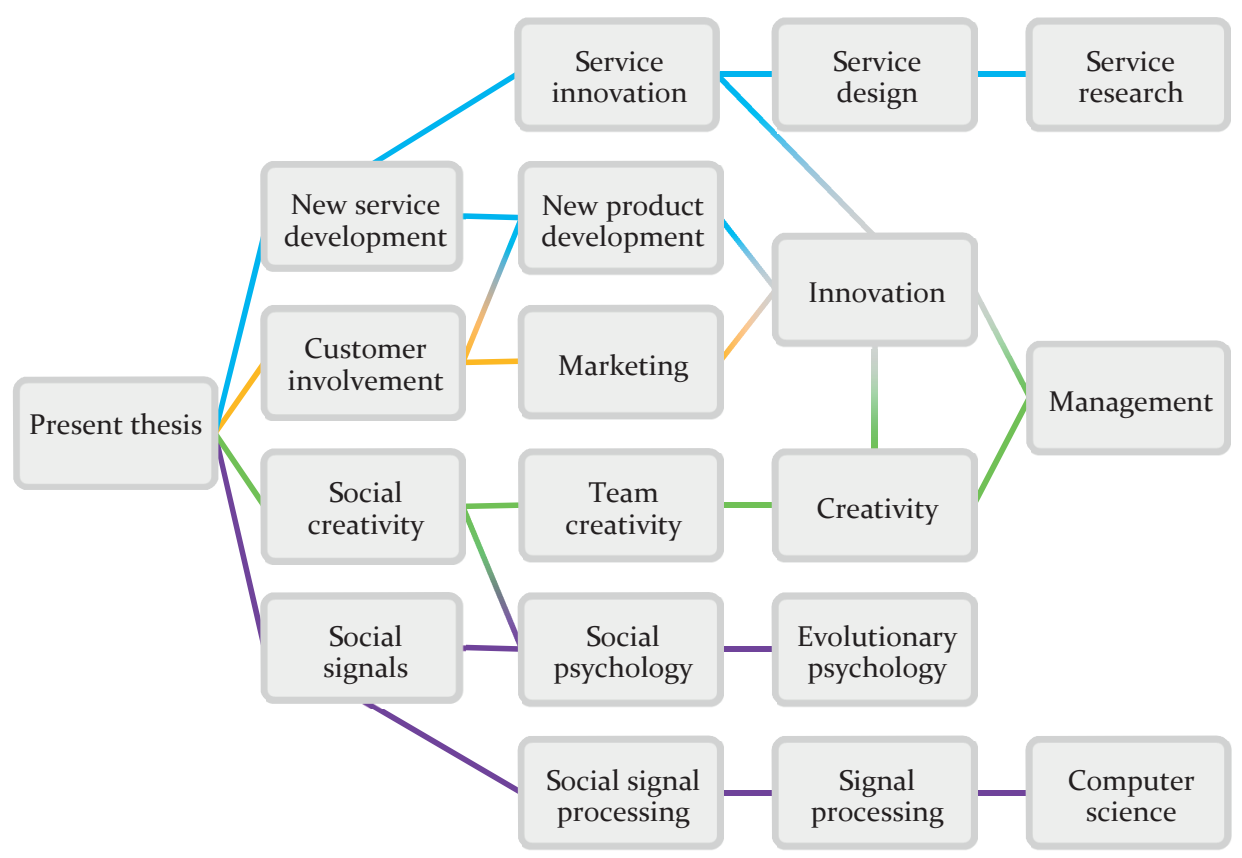

Figure 1. Depiction of constituent literature streams

Figure 1 above depicts the present thesis developing from the three fundamental literature streams, namely (1) service research (which informs the content on service development processes), (2) management (which provides the foundation for customer involvement as catalyst for innovation and for team dynamics and creativity), and (3) computer science (which informs the deployment of wearable technology as well as the algorithms for analysis). We elaborate on the first two literature streams in the sections below; chapter 2 is dedicated to an in-depth exploration of the third literature stream.

\section{Unraveling New Product Development, New Service Development, and Service Innovation}

Coombs and Miles's (200o) taxonomy comprises three contiguous perspectives in service innovation research: assimilation, demarcation, and synthesis. The assimilation perspective posits that the insights developed for product innovation research are appropriate as such to study service innovation as long as the innovation drivers are technology-related (Gallouj, 2002; Tether, 2005; Toivonen \& Tuominen, 2009). By contrast, the demarcation approach calls for a stringent focus on the particulars of service innovation (e.g., intangibility, prerequisite for customer integration, etc.) in terms of both output and processes (e.g., Coombs \& Miles, 2000; Drejer, 2004; Hipp \& Grupp, 2005). The neo-Schumpeterian synthesis 
perspective calls for an integrative innovation theoretical lens to comprise both services and manufacturing beyond technological innovations focusing on the emergence of new combinations that improve (innovate) previous solutions (e.g., Drejer, 2004; Flikkema, Jansen, \& Van Der Sluis, 2007; Sundbo, 1997; Toivonen \& Tuominen 2009; Witell, Snyder, Gustafsson, Fombelle, \& Kristensson, 2016).

Some scholars consider 'service innovation' and 'new service development' synonymous and define them as a "[the] process of devising a new or improved service, from idea or concept generation to market launch" (Biemans, Griffin, \& Moenaert, 2016, p. 383). The new service development (NSD) literature often uses the two terms interchangeably (Menor, Tatikonda, \& Sampson, 2002). Other scholars oppose this view, as one should not separate the process of developing new services from the implementation and value creation of the new services (Skålén, Gummerus, von Koskull, \& Magnusson, 2015); thus, they extend the definition of service innovation to include NSD as a complimentary stage of service innovation. When assessing the success of service innovation both the synonymy as well as the complementarity perspective, however, create confusion since it is not clear if one refers to the successful developing process or its outcome (Witell et al., 2016). As to allow for a discrete evaluation, I thus argue that "new service development" and "service innovation" should be conceptualized and assessed separately, the former as the process and the latter as the outcome to be evaluated as such upon adoption.

One of the indirect implications of Coombs and Miles's (2000) taxonomy is that the theoretical support developed for new product development (NPD) is broadly inapplicable for understanding NSD. Even though NSD originated as a spin-off from NPD, services scholars argue that it has now reached subject specialty (Biemans et al., 2016), but it still requires building the field from the ground up beginning with unbiased qualitative research to explore the specific nature of NSD, thus departing from applying NPD frameworks to the NSD context. Scholars researching this field have yet to synthetize an NSD growing body of knowledge that explains fundamental phenomena such as what the process looks like and how it can be effectively managed (Biemans et al., 2016). Other scholars, whose views we adhere to, believe that the fundamental distinction between products and services is outdated and further state the ubiquitous character of services (Rust \& Huang, 2014).

\section{Customer Involvement in NPD/NSD}

Firms seek to acquire knowledge they may lack internally about their customers' needs as well as solution-related information (Nambisan 2002; Poetz \& Schreier 2012). Thus, firms invite customers to provide input about their needs and preferences as well as to suggest new ways to solve problems (Poetz \& Schreier, 2012). Customer involvement refers to a customer's participation in the firm's NPD process (Fang 2008; Ommen, Blut, Backhaus, \& Woisetschläger, 2016; Storey \& Larbig, 2018). This type of involvement enables firms to circumvent delays that could stem from a mismatch between customers' ideas and needs, thus helping firms to timely design, develop, and lunch new innovation opportunities (Carbonell, Rodríguez-Escudero, \& Pujari 2009). 
The time pressure is particularly relevant as the impact of customer participation on NPD performance is directly proportional to the technologically turbulence of the NPD projects, thus the speedy access to customers' need- and solution-related knowledge is crucial (Chang \& Taylor 2016). A proficient knowledge managing process (i.e., acquired through customer participation) is dependent on the quality of the provided insights from the knowledge provider (i.e., customers) (Mahr, Lievens, \& Blazevic 2014), the actors' impetus and capabilities (i.e., customers and firms') related to the knowledge exchange process, and the knowledge seeker's (i.e., firms') capacity to efficiently gather the creative insights and morph them into tangible outcomes (Szulanski, 1996; Von Hippel 1994). In other words, customer involvement is the process employed by companies of acquiring external knowledge directly from customers on their needs and solutions and integrating said knowledge into the firm's innovation process (Chang \& Taylor 2016). In their meta-analysis, Chang and Taylor (2016) hypothesized that the relationship between customer involvement and NPD performance is stronger for developing services than for developing goods. They however did not find any evidence to support their assumption. In addition, recent empirical studies find that, when it comes to the outcomes of customer involvement, there is no significant difference across industries. In other words, involving customers in the development of new products and services produces similar results, such as the improvement of innovation performance by refining their knowledge on customers' needs and thus developing solutions tailored to those needs (Cui \& Wu, 2015). We can conclude that, in the new product development literature, within the boundaries of customer involvement one can apply the tools developed for assessing NPD to the development of new services.

\subsection{Types of Customer Involvement}

Customer participation (or involvement) activities in NPD do not have an exact blueprint especially due to the different roles customers are invited to play in the process. More specifically, Fang (2008) classifies customer roles across two dimensions: as an information resource (CPI) and as a co-developer (CPC). Relatedly, Cui and Wu (2015) depict three categories of customer participation: as an information source (CIS), as co-developers (CIC), and as innovators (CIN). CIS and CIC are homonymous to Fang's (2008) taxonomy, the third (CIN) places the 'locus of control' in the realm of the customer (e.g., a crowdsourced platform such as Threadless where the customers design and vote the best products that then the company produces and sells) (Baldwin \& von Hippel, 2011). The CIS type requires minimal involvement from the customer's end and the locus of control of the implementation resides completely with the company. A straightforward example of CIS is the market research or actively inviting the customers to share insight, needs, and wants that could improve the company's offering. The CIC type entails an active role of the customers and a shared effort in developing the product. Conceptually, CIC is closest to "co-design" or "co-creation" as theorized in the service literature. It is crucial to make a clear distinction between them. Service scholars often use "co-creation" to denote any activities that utilize customers' creative inputs in the service design or delivery process. However, CIC only refers to settings in which customers work closely with NPD employees to jointly develop new solutions (Cui $\& \mathrm{Wu}, 2015$ ). Examples of $\mathrm{CIC}$ are consultancy firms (i.e., professional service providers) that co-develop solutions for and with their B2B customers. 


\subsection{Involvement of Consumers $\left(\mathrm{B}_{2} \mathrm{C}\right)$ and Customers $\left(\mathrm{B}_{2} \mathrm{~B}\right)$}

Aside from the specificity of the role, there are also two types of interactions firms undertake, namely with their consumers (in business-to-consumer markets) and/ or with their customers (in business-to-business markets). These two different types of interactions also render discrete outcomes. Inviting consumers to join the NPD process produces lower performance than involving business customers. More specifically, the $\mathrm{B}_{2} \mathrm{~B}$ customer involvement in the NPD process decreases the new product's time to market and consequentially enhances its financial performance with up to $65 \%$ compared to consumers' contributions (Chang \& Taylor, 2016).

New product development literature suggests few explanations for these meta-analytic findings. From a knowledge provision perspective, the motivation to contribute to the innovation process in an active manner varies between $\mathrm{B}_{2} \mathrm{C}$ and $\mathrm{B}_{2} \mathrm{~B}$ contexts as well as their respective ability to possess knowledge both reliable and relevant (Afuah, 2003; Bogers, Afuah, \& Bastian, 2010).

In practice, the scale and growth of the $\mathrm{B}_{2} \mathrm{~B}$ market makes the outcomes of customer involvement even more important. The European consulting market (i.e., technology, HR, operations, and strategy consulting as well as financial advisory) has witnessed a consistent growth since 2010 approaching $\$ 100$ billion for the first time in history representing $41 \%$ of the global consulting market in 2016 (of a total value of \$251 billion) (Statista, 2018). Moreover, with the industry boundaries' blurring (Consultancy.uk, 2018; IBM, 2016) the competition becomes even more fierce. The $\mathrm{B}_{2} \mathrm{~B}$ market is disrupted and being taken over, on one hand, by design firms that acquire know-how in organizational change and advanced technology, and, on the other hand, by consulting firms that shift to systematically incorporating design thinking methodologies (Consultancy.uk, 2018).

Compared to the $\mathrm{B}_{2} \mathrm{C}$ market, the higher motivation of business customers can be explained by the greater number of explicit benefits from sharing their knowledge in the NPD process such as highly customized solutions, involvement in the selling process, and exclusive privileges to said customized solutions for a period of time (Brockhoff, 2003). Regarding the reliability and relevance of the possessed knowledge, $\mathrm{B}_{2} \mathrm{~B}$ customers tend to have a higher level of shared language and understanding of needs, which makes them better partners in reaching the NPD project goals relative to consumers (Mahr et al., 2014). Subsequently, business customers can produce more appropriate and viable ideas to solve the NPD project brief (Gargiulo \& Benass, 200o). The same stream of literature cautions about the downsides of the $\mathrm{B}_{2} \mathrm{~B}$ customer involvement such as potentially sharing information that is neither diverse nor authentic (Gargiulo \& Benass, 200o), or creative possibly due to business customers' tendency toward convergent thinking styles and strategic deliberation (Chang \& Taylor, 2016). To date there is no evidence of how the interaction and exchange of ideas directly affects the NPD process (Cui \& Wu, 2017).

\section{Creative Problem Solving}

Conceptualized as the inception of innovation, creativity is the generation of new and valuable ideas in context (Amabile, 1996; van de Ven, 1986). This idea generation process is 
task dependent and current literature distinguishes between three types of creative pursuits namely (1) creative storytelling or verbal creativity, (2) artistic creativity, and (3) creative problem solving (Amabile, 1996). Evidently, the development of a new offering requires mostly identifying and appropriately addressing customer's needs hence the third type of creativity is the focus of the following chapters.

Although defined as the process of creating new and valuable ideas, most scholars operationalize the concept of innovation as a static outcome variable (e.g., Ahuja, 2000; Baer, Evans, Oldham, \& Boasso, 2015; Hülsheger, Anderson, \& Salgado, 2009). Thusly, there is no consensus in the literature as to what extent creativity as an ideation process brings about innovation (Anderson, De Dreu, \& Nijstad, 2004; Rosing, Frese, \& Bausch, 2011). We propose that creativity is the process of generating new and useful ideas and that the output of this process is a new innovative service. The source of creative input has long been considered to be stemming solely from the individuals involved in the creative process (Bharadwaj \& Menon, 200o; Scott \& Bruce, 1994). More recently, a growing body of literature revised this perspective and began examining how the dynamic social interactions shape the creative process (Brennan \& Enns, 2015; De Jaegher, Di Paolo, \& Gallagher, 2010; Heerey, 2015). This new perspective recognizes the importance of the individuals' contribution but stresses that the individual creative potential sparks in the (social) interaction between team members (Perry-Smith \& Shalley, 2003). However, there is still an essential gap in the literature on how the role of the social interactions varies throughout the stages of creative pursuits (generation, elaboration, and implementation) (Perry-Smith \& Shalley, 2014; Cui \& Wu, 2015). Returning to the LEGO lightbulb metaphor, in the context of developing a new service, the co-developing $\mathrm{B}_{2} \mathrm{~B}$ team is no different than any other team when it comes to verbal and nonverbal interaction and how they engage in a stochastic combinatorial process of ideas (Simonton, 2003).

In sum, customer involvement is a catalyst for innovation. Involving customers as 'codevelopers' entails closely working with them as temporary members of the NSD team. Customers' input is crucial because it brings in new project relevant knowledge about their needs and potential solutions. The output is dependent on the interaction among team members. Here, we aim to shed light on the team dynamic by looking at the team's communication channels and nonverbal cues (social signals) (Pentland, 20o8; Perry-Smith \& Shalley, 2014; Roudposhti, Nunes, \& Dias, 2016) as well as the communication content (creativity) (Amabile 1983, 1996; King \& Andersen, 1990). We thus also aim to answer the following research question: how do team interaction patterns affect team creativity throughout the new service development project process?

\section{The Objectives of the Dissertation}

The motivation and foundation of this thesis can be summarized in one word: "betterment". Firstly, this applies to the conceptual and theoretical perspective. Innovation through customer involvement is the way of making better services, addressing customers' needs in a better way, co-developing win-win-win solutions for all involved stakeholders of the innovation process. Secondly, this applies to the new way of collecting data. I argue that the following chapters describe a better way to investigate the new service development process 
and set a methodological stepping stone into deploying better measurement tools that collect objective data for advancing knowledge in the NSD field, but also broadly in the innovation and management literature.

\section{The Structure of the Dissertation}

The dissertation continues as follows. Chapter 2 presents a method addendum whereby we address calls in for the advancement of the field of team research through the deployment of new technologies for data collection; the chapter also serves as a methodological precursor for the following two chapters. Chapters 3 and 4 tackle the micro-level processes of nonverbal behaviors conducive to creative problem solving in the context of new service development by $\mathrm{B}_{2} \mathrm{~B}$ project teams; also, both chapters focus on the collaboration dynamic between professional service providers engaging their $\mathrm{B}_{2} \mathrm{~B}$ customers in the development of the new offering. A detailed description of the dissertation is presented in the following sections and in Table 1 below.

\section{Summary of Chapter 2: Capturing the Right Signals: Method Addendum}

The chapter discusses recent advancements in team science research designs. Following the research calls in the field we provide an overview of new types of data for social science research suitable for answering said calls (i.e., social signals). The chapter then explores processing tools and machine learning algorithms as viable methods of analysis of these new data. We then discuss data-driven theory development as a viable alternative for inductive or deductive theory building paradigms. The chapter concludes with a step-by-step best practice procedure of conducting a study with wearable devices and dealing with such new types of data.

\section{Summary of Chapter 3: It Takes Two to Tango}

The premise of this chapter is that both entities (i.e., the service provider and their $\mathrm{B}_{2} \mathrm{~B}$ customer) need to be present and in close interaction to best tackle the development brief for the first phase of the development process. We considered the time the team members spent in close proximity, the communication channels they formed, and their body language as factors shaping creative outcomes in this new service development (NSD) team. The foundation of this chapter is the set of network structures they create while being around each other and communicating during the project meetings. As such structures are relatively well understood in basic terms, in this study, we use two contrasting analytical techniques to demonstrate that preliminary evidence does exist in support of social signals as markers of creative success within teams. We computed multiple regressions with the quadratic assignment procedure (MRQAP) with state-of-the art multi-class classification revealing the roles of different objective measures gathered using wearable computing devices.

\section{Summary of Chapter 4: For Your Ties Only}

The initial assumption of this chapter was that some relationships (i.e., ties) are more important for the creative process than others during the new service development process. 
This study considers one more social signal than chapter 2, namely the voice characteristics of team members. We introduce the concept of "creative activation" as the markers of convergence between creative ties and social signals. We set out to understand team interaction patterns (generated by two new service development (NSD) teams, and their participating customers during actual company meetings) that lead to creative problem solving by looking at how creative ties align with objective social signals over the entire project span. We employ a state-of-the art dynamic network alignment method that facilitates the formalized comparison of networks with common constituent node and edge types, but different nodes within these types, and different topological structures. The outcome of this chapter is that we (a) show network topological evidence of creative activation in teams, and, (b) isolate patterns in social signals (i.e. interaction synchrony) at different points in time that contribute most to this result.

Table 1. Overview of empirical studies

\begin{tabular}{|c|c|c|}
\hline & $\begin{array}{c}\text { Chapter } 3 \\
\text { "It Takes Two To Tango" }\end{array}$ & $\begin{array}{c}\text { Chapter } 4 \\
\text { "For Your Ties Only" }\end{array}$ \\
\hline Research question & $\begin{array}{l}\text { How do social signals drive } \\
\text { reciprocal creativity? }\end{array}$ & $\begin{array}{c}\text { How do social signals align with } \\
\text { dyadic creativity? }\end{array}$ \\
\hline Theoretical lens & \multicolumn{2}{|c|}{ Signaling theory (Connelly et al., 2011) } \\
\hline $\begin{array}{l}\text { Creativity } \\
\text { assessment }\end{array}$ & $\begin{array}{c}\text { Reciprocal } \\
\text { (alter self-assessed) }\end{array}$ & $\begin{array}{c}\text { Relational } \\
\text { (dyad self-assessed) }\end{array}$ \\
\hline $\begin{array}{l}\text { Type of customer } \\
\text { involvement }\end{array}$ & \multicolumn{2}{|c|}{ Customer as co- developer (CIC) } \\
\hline $\begin{array}{l}\text { B2B context } \\
\text { (industry) }\end{array}$ & $\begin{array}{l}\text { Recruiting } \\
\text { service provider }\end{array}$ & $\begin{array}{l}\text { Healthcare devices manufacturer; } \\
\text { Graduate education }\end{array}$ \\
\hline Team composition & $\begin{array}{c}\text { Joint B2B NSD team } \\
\text { (consultancy and customer) }\end{array}$ & $\begin{array}{l}\text { Joint NSD project team } \\
\text { (consultancy, customer, and } \\
\text { experts) }\end{array}$ \\
\hline Team type & \multicolumn{2}{|c|}{ Physical project teams } \\
\hline $\begin{array}{l}\text { Teams in the } \\
\text { sample }\end{array}$ & 1 & 2 \\
\hline $\begin{array}{l}\text { Participants per } \\
\text { team }\end{array}$ & 14 & $\begin{aligned} \text { Team } G & =19 \\
\text { Team } M & =16\end{aligned}$ \\
\hline Data collection & \multicolumn{2}{|c|}{ Longitudinal (self-assessed) survey and sociometric badges } \\
\hline Unit of analysis & Task & 30-minute meeting episodes \\
\hline $\begin{array}{l}\text { Temporal } \\
\text { perspective }\end{array}$ & 29 tasks in 3 meetings & $\begin{array}{c}\text { Team } \mathrm{G}=78 \text { episodes in } 8 \\
\text { meetings } \\
\begin{array}{c}\text { Team } \mathrm{M}=64 \text { episodes in } 9 \\
\text { meetings }\end{array}\end{array}$ \\
\hline Sample size & 145 matrices $=>20710$ dyads & $\begin{aligned} \text { Team } G & =4211 \text { dyads } \\
\text { Team } M & =3455 \text { dyads }\end{aligned}$ \\
\hline Dataset structure & $\begin{array}{l}\text { Global unfolded multiplex } \\
\text { networks and dyadic data }\end{array}$ & Dyadic data \\
\hline Methodology & $\begin{array}{l}\text { Multiple regression using the } \\
\text { quadratic assignment procedure } \\
\text { (MRQAP in UciNET) and } \\
\text { WEKA Multiclass Classifier }\end{array}$ & $\begin{array}{c}\text { Multiple pair-wise network } \\
\text { alignment } \\
\text { (GraphCrunch2) }\end{array}$ \\
\hline
\end{tabular}




\section{Theoretical Lens}

Signaling theory (Spence, 1973) was first put forth in the economics literature to explain how certain behaviors carry signals that reduce information asymmetry between two parties who previously had access to different information (Connelly, Certo, Ireland, \& Reutzel, 2011), namely the sender and the receiver of the signal (Spence, 2002). The theory was first used to explain the signaling function of education on the labor market. Specifically, employers do not have any a priori way of differentiating between high- and low-performing potential employees; a candidate holding a higher education degree is signaling to employers that they are better performing than those without such degree (Spence, 1973). Without this signal that balances the previous information asymmetry, the recruiting process would be very inefficient for the hirer (Arkerlof, 1970). The theory has been applied to a vast plethora of literature on signals and their mechanisms from zoology and anthropology (Bird, Smith, Alvard et al., 2005) to the management literature (Connelly et al., 2011). Signaling theory (Connelly et al., 2011) offers a meaningful theoretical lens for revealing behavioral collaboration patterns in the case of information asymmetry (Kao and Schmandt, 2015; Liu \& Donath, 2006). We highlight the parallel between the notions of macro-level signaling (Connelly et al., 2011), for example in the market place as a basis for understanding the microlevel social signals given away by the collaborating parties. Just like how at macro-level one party is concerned about another party's behavior or behavioral intentions (Elitzur \& Gavious, 2003), at micro-level the collaborating entities start from a state of information asymmetry and need to adjust the interplay of interactions to uncover latent knowledge (Connelly et al., 2011), needs, and ideas to best develop a new offering. More specifically, the theory will explain what behavioral cues are sent (or "social signals" such as vocalics, proxemics, and kinesics) and how the receivers respond behaviorally to them (Pentland, 2008). 


\section{Chapter 2}

Capturing the Right Signals: Method Addendum 


\begin{abstract}
The motivation of this chapter is to address calls for the progression of the field of team research through the application of new technologies for data collection (Salas, Reyes, \& McDaniel, 2018). Specifically, we provide (1) background on recent advancements and primary considerations in team science research designs, (2) an overview of social signals as a new type of data for social science research, (3) a summary of social signal processing as a mean of collecting this new type of data, (4) a synopsis of machine learning based methods of analysis of new data complemented with (5) establishing 'ground truth' measures (a fundamental concept in machine learning and a particularly important pillar in translating social science theory into quantified insights into human behaviors). Then, we discuss (6) data-driven theory development, as contrasted and aligned with the development of valid and parsimonious models of social behavior based on social signals. We conclude with (7) an example of a step-by-step best practice of conducting a study with wearable devices and dealing with such new types of data.
\end{abstract}




\section{Background}

Recent calls for research on dynamic constructs (e.g. creativity, idea evolution, and codevelopment) indicate the upper limits of cross-sectional designs and static assessments as advancing the field of team dynamics requires intensive longitudinal designs be them as resource intensive, difficult, and risky as they may (de Montjoye, Stopczynski, Shmueli, Pentland, \& Lehmann, 2014; Hennessey \& Amabile, 2010; Kozlowski, 2015; Perry-Smith \& Shalley, 2003). One of the main restrictive factors in capturing process dynamics is the hegemony of questionnaire-based measurements in research designs (i.e., questionnaires are obtrusive, they interrupt the natural stream of behaviors, they are time consuming, they are fundamentally inadequate for high frequency sampling rates, they are prone to response biases and these biases are enhanced by repeated measurements). Surveys are indeed essential for measuring psychological constructs, but they are also limited slices of behavior that we can complement with observable and objective behaviors for probing the dynamics frontline.

Thus, a first step in the direction of developing timely and appropriate research designs in team dynamics research broadly, is to complement traditional tools for data collection (i.e., questionnaire based measurements) with new assessment tools. Hitherto, researchers suggested many applications of the technology in computational social science (e.g. Bersin, Mariani, Monahan, 2016; George, Osinga, Lavie \& Scott, 2016; Montanari, Nawaz, Mascolo, \& Sailer, 2017), but this research has mainly been descriptive in nature, providing guidance as to how to capture team interactions with rich data (Kim, McFee, Olguin, Waber, \& Pentland, 2012).

\section{Social Signals - "New" Types of Data}

A “'social signal' [...] is a communicative or informative signal that, either directly or indirectly, conveys information about social actions, social interactions, social emotions, social attitudes and social relationships" (Poggi \& Francesca, 2010, p. 1). The "social" in "social signals" pertains to the type of information conveyed by the signal; in other words, said signal carries social information that regulates the social relationships between emitter and receiver. It does not mean, however, that the signals are social because they are shared with others, socially (Idem). We emit visual social signals through our nonverbal behavior such as body language and gestures, facial expressions (Baur, Damian, Lingenfelser, Wagner, \& André, 2013), and relative location from others (Poggi \& Francesca, 2010). Another type of social signals (i.e., audio) pertains to speech and voice patterns which can be best explained by the common anecdotal phrase "it's not what you said, but how you said it" (Frank, Griffin, Svetieva, \& Maroulis, 2014). Consequentially, communication success depends on explicit content but also on the implicitly conveyed social signals (Baur et al., 2013).

Research on social signals has largely focused on understanding how receivers perceive emotions and intentions (Loth \& De Ruiter, 2016). Also conceptualized as "affective signals", social signals as expressions of emotion give social cues regarding others individuals' interest, empathy, and emphasis (Roudposhti et al., 2016), help individuals understand others' attitudes by their gestures (Pease \& Pease, 2016), when emitted by groups of individuals they facilitate the rapid adoption of adaptive behaviors in dangerous situations (de Borst \& Gelder, 
2016; Ekman, 2004; Martinez, Falvello, Aviezer, \& Todorov, 2016), and have the potential of assisting the redesign of human-machine interactions (Loth \& De Ruiter, 2016). The fundamental importance of social signals is that from short exposures to human behavior (as short as $\mathbf{2 5 0}$ milliseconds) we have the capacity to accurately identify emotions above the chance level (Martinez et al., 2016). Moreover, research shows that our innate ability to understand someone's unfamiliar or ambiguous signals even transgresses one's cultural boundaries (Earley \& Mosakowski, 2004). The capacity to understand emotions through these short exposures to or "thin slices" of behavior have received significant attention for more than two decades (Ambady, Bernieri, \& Richeson, 2000; Wilson \& Schooler, 1991).

\section{Social Signal Processing - Capturing and Dealing with New Types of Data}

The field of computer science defines '(digital) signal processing' as the technology enabled process that covers the theoretical foundations, applications, and implementations of processing information enclosed in different physical, symbolic, or abstract formats broadly designated as signals (Moura, 2009). In the same research stream, a signal is defined as physical manifestation of information (e.g., audio, speech, video, language, image, multimedia, sensor, communication, geophysical, sonar, radar, biological, chemical, molecular, genomic, data, or sequences of attributes, or numerical quantities, etc.). Processing such signals comprises the operations of discovering, recording, representing, or reproducing signals by analyzing and interpreting (Basseville, 1989; Moura, 20o9; Rabiner \& Gold, 1975).

Revered for their expertise in signal processing and inspired by the literature on "thin slices" in social psychology (Ambady, Bernieri, \& Richeson, 200o; Poggi \& Francesca, 2010), computer scientists have developed the new area of social signal processing (SSP). Introduced by the pioneering works of Alex "Sandy" Pentland and his group at MIT Media Lab (Vinciarelli, Pantic, \& Bourlard, 2009), SSP sits at the conceptual intersection of social signals and signal processing. It can thus be defined as the technology enabled process of detecting and recording information about social actions, interactions, emotions, attitudes, and relationships by digital (wearable) devices (i.e., firmware). Their first cutting-edge wearable device (namely the "sociometer" or the "sociometric badge") (Kim et al, 2012; Pentland, 2008; Tripathi \& Burleson, 2011; Waber, Olguín-Olguín, Kim, Mohan, Ara, \& Pentland, 2007) was designed to automatically detect and collect social signals in dyadic interactions such as vocal social signals and the body movement and energy signals (Vinciarelli et al., 2009). The second generation of the devices detect and record a wider plethora of social signals (i.e., vocal social signals, posture and body movement, time spent in relative proximity and in face-to-face interactions) (Olguín-Olguín, 2007). Their incipient findings present predictions made with an accurateness of more than $70 \%$ on outcomes of salary negotiations, hiring interviews, and speed-dating dialogues (Pentland, 2008; Vinciarelli et al., 2009). Other SSP endeavors uncovered social constructs such as mimicry (Chartrand \& Bargh, 1999; Vinciarelli et al., 2009), "honest signals" detection (Pentland, 2008), agreement and disagreement (Bousmalis, Mehu, \& Pantic, 2009), dominance (Poggi \& D’Errico, 2010), backchannel and floor management (Poggi \& Francesca, 2010). The SSP literature now predominantly addresses the effect of more varied nonverbal cues (i.e., facial expressions and gazing, body posture and gestures, vocal characteristics, relative distances 
in the space, etc.) on social interactions (Cristani, Raghavendra, Del Bue, \& Murino, 2013). Even though the SSP field is in its inception phase, it has already attracted the consideration of the technological and research communities (Bersin et al., 2016; Kozlowski, 2015). Mining the objective social signals (also referred to as "reality mining") is placed in the top ten methods that have the potential of changing the world, management experts expecting SSP to be for organization studies the change the microscope brought to medicine decades ago (Buchanan, 2007; Vinciarelli et al., 2009). There is consensus on how impactful the technology could be, but research employing the technology in social sciences is lagging behind as it has mainly been descriptive (Kim et al., 2012).

\section{Machine Learning Algorithms - "New" Tools for Analyzing "New" Data}

The "traditional approach" to collecting and analyzing data in social sciences comprises a series of set steps: (1) positioning within a theoretical field, (2) theoretical framing and hypotheses development, (3) selecting the appropriate research context to conduct the study, (4) data collection and dataset curation, (5) hypotheses testing (considering model parsimony, controls, heterogeneity, etc.), and (6) results' discussion and study's contributions. In other words, in this traditional statistics paradigm, given an applied problem one must choose a good parametric class of models that integrates our knowledge of the system (Bzdok, Altman, \& Krzywinski, 2018) to fit the data onto with the aim of achieving generalizability, subsequently estimating parameters and then drawing conclusions (e.g., Blei \& Smyth, 2017; Breiman, 2001b; Wood \& Gorman, 1971).

However, especially when dealing with high dimensionality, if the models are a poor representation of reality, the conclusions could be erroneous. One approach to addressing these concerns is to test the data model fit using goodness-of-fit tests and residual analysis (e.g., Breiman, 2001b). However, such standard tests reject linearity only when the nonlinearity is extreme and they have limited power unless the direction of the alternative is accurately specified (Bickel, Ritov, \& Stoker, 2006). These tools are ideal for the purpose they were meant to serve, when a data model is the best available solution to a data problem. However, when facing a wider array of data problems, we need a more diverse toolset (Blei \& Smyth, 2017; Breiman, 20o1b; Munoz, Villanova, Baatar, \& Smith-Miles, 2018; Trevor, Robert, \& JH, 2009; Zhou, Pan, Wang, \& Vasilakos, 2017).

When dealing with studies (1) designed at the intersection of multiple research fields, whereby (2) the theoretical framing does not provide sufficient input for developing a system of relationships among construct but we have an intuition of what theories and mechanisms are at play we need a "less-traditional approach" (for social sciences) to analyzing data. Machine learning (ML) is such an approach for studies when the theoretical foundations are insufficient for constructing hypotheses but the structure and complexity of the data are known. Additionally, for longitudinal data, when theoretical precedent for the temporal dimension of a research question is scarce, employing ML algorithms allows for data to be handled first in a static manner to establish separability of targets or events as an investigative starting point (Montanari, Tian, Francu, Lucas, Jones, Zhou, \& Mascolo, 2018).

Related to the fields of human learning, neuroscience, organizational behavior among others, the field of ML studies inference under uncertainty. With the rise of mobile devices 
and wearable computing in the past fifteen years (Callegaro \& Yang, 2018; Swan, 2013), scalable ML procedures (combining computational and statistical considerations) have been developed to innovatively investigate high throughput of data (Jordan \& Mitchell, 2015; Teng, 2016). These devices collect large amounts of data of high granularity and specificity; this lead computer scientists, engineers (Montanari et al., 2018), and, most recently, social scientists (Dietzel, Francu, Lucas, \& Zaki, 2018) turn to ML to infer predictions (i.e., predictive analytics) and make decisions from these "modern" data-sets (Jordan \& Mitchell, 2015).

ML tasks are broadly categorized in two types. If the learning system is left on its own to explore the data and determine structures within, the task is called "unsupervised machine learning". This applies to cases when we don't know anything about the structure and the properties of the data and aim to explore structural patterns. If the learning system is provided with a learning procedure (i.e., training) that will enable it to map labeled inputs onto outputs (i.e., testing), the task is known as "supervised machine learning" (Flach, 2012). This approach establishes dependencies and determines models based on mapping relationships among variables in the data-set. A widely-used supervised ML task is classification (Idem). In broad terms, in such problems the data comprises two types of classes, namely input and output. This could mean a dataset comprising (1) several input classes and two output classes (also referred to as binary classification), or (2) multiple input as well as output classes (also referred to as multi-class classification") (Flach, 2012).

In a simple sense, classification uses training and testing to boost the generalizability of findings. This method is suitable for scenarios when we know the structure of the data and the theoretical underpinnings of the variables but it is too early in theorizing to establish $a$ priori whether the relationship between variables is linear or polynomial. In this domain, the diverse needs of different applications thus necessitate a wide range of mathematical structures to provide flexible trade-offs between the volume of data, computational complexity, and performance, diversifying the range of possible learning architectures (Breiman, 2001a, 2001b; Jordan \& Mitchell, 2015).

Even though synergies with related fields have occurred over the past fifteen years, we are only at the early stages of experimenting with ML methods across fields and of deploying them in social science. In Chapter 3 of this dissertation, we use a reputable and generalpurpose algorithm to uncover which social signals best predict creativity at dyadic level. Similarly, Chapter 4 demonstrates an implementation of an algorithm developed for and implemented to date solely in the field of bio-informatics (namely, in research on aligning graphs determined by protein-to-protein interactions) (Djeddi, Yahia, \& Nguifo, 2018); we apply the method to graphs described by the social signals collected with wearables to uncover similar pattern alignment which could not have been revealed with traditional computational or statistical methods.

\section{Self-Reports as Ground-Truth for Classification}

As extensively covered in the previous section, the difference between statistics and ML lies primarily in their respective objectives. The former allows to test hypotheses on a representative sample with the aim of testing a generalizable model (based on theoretical 
background, context, etc.); the latter distils the model from theoretically informed variables and extracts the relationships between them with the objective of achieving a model with high accuracy. In the context of this dissertation specifically, we apply ML to uncover to which extent certain social signals predict creativity in teams. The objective of ML here is to achieve high accuracy of prediction given the targets (i.e., the different levels of creativity as experienced and reported by participants).

In brief, the case for the theoretical underpinning is made by the fact that we can infer from theory that the different levels of creativity should be identifiable from social signals given that such nonverbal behaviors have been proven to influence communication content and interlocutors' affects (e.g., Mehrabian \& Friar, 1969) that in turn could influence creativity (e.g., Fong, 2006).

Regarding our target variable, to date, the best way to assess creativity is through selfassessments (e.g., Amabile, 1983, 1996; Amabile \& Pratt, 2016; Cromwell, Amabile, \& Harvey, 2018; Ruscio, Whitney, \& Amabile, 1998). In both studies presented in Chapters 3 and 4 respectively we deploy surveys to collect team members' perceived creativity levels. We use these subjective data as ground-truth.

Introduced in the field of geographical and geological sciences (e.g., Pickles, 1995), the term ground-truth was defined as a measurement validation "on the ground". It has been adopted into the computer science literature to describe data that is "known" or has been proven to be correct. The concept is currently prevalent in the fields of biometrics, computer vision (e.g., Manjunath, Ohm, Vasudevan, \& Yamada, 2001), and in the development of wearable computing (e.g., Montanari et al., 2018). A variation of the term, namely ground-truth error is, too, widely used proving that what we "know" is not irrevocably accurate. In our case, even though self-assessments are the gold standard to date, new "objective" creativity measures will most dramatically decrease the ground-truth error of our current creativity measurement.

\section{6. “Abduction": Data-Driven Theory Construction}

The traditional approach to representing relationships between variables is to employ theory-based models; these models capture cause-effect relationships among variables that have been previously theoretically deduced or empirically proven. Conversely, an alternate approach is to train a "learnt model" with input features and predictive targets to automatically extract the relationships amongst them (Karpatne, Atluri, Faghmous et al., 2017).

These data structures that have been induced computationally can put under the microscope previous conjectures or established cause-effect relationships among variables. Such radical data-driven evidence is thus challenging social scientists' creativity in generating new inferential processes leading to new hypotheses and theories. In the sociology literature, this process is known as "abduction" (Timmermans \& Tavory, 2012) which is epistemologically closest to grounded theory (Bryant \& Charmaz, 2007; Haig, 1995; Kelle, 2010; Reichertz, 2007; Strübing, 2007). 
In the specific case of this dissertation, the core constructs investigated have underdeveloped causal foundations. Even though social signals have been extensively researched, the theoretical linkages with their referents or proxy-variables are lagging behind. The literature establishing causality between the social signals and interaction outputs is dispersed and non-consensual. We thus adhere to the "abduction paradigm" by employing careful methodological approaches and existing theory to elicit new theoretical insights (Timmermans \& Tavory, 2012).

\section{Capturing the Right Signals: Conducting Studies with Sociometric Badges}

As introduced in Chapter 1 , the second goal of the dissertation is to introduce a novel way of investigating the customer involvement process by employing better alternatives to traditional data collection tools. In the previous sections of this chapter I extolled the merits and benefits of deploying wearable devices to collect high quality data at high sampling rates and with accuracy for the study of human behavior. To contextualize these explanations, I will now provide a step-by-step guide on conducting a study with such devices, namely the sociometric badges.

Identical to "traditional data collection", upon deciding the purpose of the study one selects the most appropriate tools for addressing the research questions at hand. For the study of social signals, the sociometric badges are tools for collecting objective behavioral data at scale; to date, these devices are the most reputable and reliable tools for collecting academicquality behavioral data, having already had received the validation of the academic community in several research fields (e.g., Kim et al, 2012; Olguín-Olguín, Waber, Kim, Mohan, Ara, \& Pentland, 2009; Pentland, 2008; Santoro, Dixon, Chang, \& Kozlowski, 2015; Tripathi \& Burleson, 2011; Waber et al., 2007).

The different types of data are collected by the built-in sensors 5 , namely (1) two microphones (one placed in the front and one at the back of the device), (2) an accelerometer, (3) a Bluetooth transreceiver, and (4) an infrared transreceiver. The first two sensors collect individual- and relational-level data, while the latter two only collect relational data. The badges will not record speech or conversational content; unless the operating system of the devices is reprogrammed to enable this feature. In this dissertation we only collected nonverbal data. Below I detail the data collected by each sensor (for a more comprehensive description of the sampling rate and data collection and storage see Chapter 3 - Appendix 1).

Firstly, the data collected by the microphones at individual level are (strictly) nonverbal speech features (i.e., volume, voice frequency and amplitude, and speaking time) collected and stored at 0.5 second intervals. To accurately differentiate one wearer's speech (collected by the back microphone) from the other's or from the ambient noise (collected by the front microphone), the differences between the back and microphones' data are later used in the data processing to distinguish between speaking time, silent time or time when one's speech

\footnotetext{
5 The explanation below is based on the Sociometric Solutions. (2013) Sociometric Badge User Manual and Sociometric Solutions. (2013) Data Collection and Export recommendations for Researchers
} 
was overlapping with others. The relational data is derived from the speech-time feature by mapping all the wearers' interlocutions over time.

Secondly, the accelerometer collects data on body movement features from the different levels of energy generated over the three axes, namely front-back, up-down, left-right with a sampling frequency of $20 \mathrm{~Hz}$. At individual level, the aggregate measure renders the wearer's general body movement over time as well as a timeline of the wearer's standing, walking, and sitting patterns. The isolated front-back measure renders wearer's posture over time. At relational level, the social signals can be processed to describe the degree of mirroring in body movement or posture over time and with what lag.

Thirdly, the Bluetooth transreceiver collects the amount of time a wearer spent nearby other people wearing a Sociometric Badge. Lastly, the infrared transreceiver time a wearer spent facing other people wearing a Sociometric Badge.

Having covered the data sensors' capabilities, this last section will provide a description of the three main stages of conducting research with the sociometric badges as per the studies conducted for this dissertation described in Chapters 3 and 4. These stages are (A) preparation for data collection, (B) mechanics of the day of the deployment, and (C) data management and preparation for analysis.

\subsection{Stage A: Preparation for Data Collection}

Step 1. First and foremost, the sociometers as well as the computer to be used for downloading and processing the data need to be set up. Preparing the badges for deployment entails updating the operating system of each device to be deployed. Also, to avoid errors in download and export in the later stages, the data management software needs to be installed on a computer and used to set up the outline of the dataset (i.e., setting the number of teams, number of participants per team, the approximate timeframes the data will be collected in, assigning a badge number to each participant, etc.)

Step 2. Alongside the technical details, this stage requires finalizing the legal documentation for the data collection. Depending on the type of study and on the institution's ethics guidelines, this could entail a comprehensive array of documents such as developing an NDA with a collaborating organization and a consent form for each of the participants in the study. The latter is the outmost minimum requirement for complying with the General Data Protection Regulation (GDPR).

Step 3. In order to ensure the appropriate distribution of the sociometers and to keep track of all distributed devices for retrieval, I have devised a distribution chart and used it for marking the handing in and out.

Step 4. The sociometers need an eight-hour charging session prior to the data collection to guarantee a full battery.

\subsection{Stage B: Mechanics of the Day of the Deployment}

Step 5. The devices need to be connected to the laptop as close to the data collection as possible to assure the synchronization of internal clocks. 
Step 6. For both projects presented in Chapters 3 and 4, before firstly deploying the devices, I have presented the technical specifications of the devices as well as the data to be collected to my participants; the information was also extensively described in the two copies of consent forms I handed out to each of my participants for signing (of which I collected one).

Step 7. After receiving the informed consent and addressing any questions, I have handed out the respective badges to each of my participants and filled in the badge distribution chart.

Step 8. At the end of the data collection, I have collected the sociometers and filled in the reception in the badge distribution chart.

Steps 7' and 8'. For longitudinal studies where deployment is recurrent (as it was the case with the studies making up this dissertation), steps 7 and 8 are repeated with each data collection.

\subsection{Stage C: Data Management and Preparation for Analysis}

Step 9. After every data collection it is recommended to back up the raw data collected.

Step 1o. After the backup, the data can be downloaded on the computer on which the dataset had previously been set up.

Step 11. Depending on the research question, the data export requires specific parameters. For instance, if the granularity requirement is high, then the temporal filter should be set for second-by-second. Also, depending on the research questions and the required level of analysis, the data needs to be exported as individual-, dyadic-, or network-level.

Step 12. Once the parameters are tuned, the data is ready for export. Depending on the computer's computing power, the level of analysis, the magnitude of the dataset, etc. this computationally intensive process could take several days.

Step 13. Some variables might require additional manipulation and/ or computations depending on the research question and variables' operationalization. For instance, in Chapter 3 we were interested in the effect of the relative amount of time spent in positive posture mirroring on creativity. Since the algorithm renders the mirroring values as they occur over time, to appropriately operationalize our variable there was a need to isolate the positive values and then calculate their percentage in relation to the entire time spent in interaction.

Step 14. For the longitudinal datasets used in this dissertation I have exported each session separately, converted the required variables, and then concatenated the resulting partial datasets into a master dataset.

Step 15. Once the dataset comprising the exported and computed social signals is completed the master dataset can be completed with the addition of the variables collected other data collection tools (i.e., surveys, coding, observations, etc). 


\section{Deploying Sociometric Badges "In the Wild"}

This chapter provided an overview of the methodological value of adopting and deploying wearable technology to advance knowledge in team science research. The high granularity and accuracy of the behavioral data collected with such sensor-infused devices, we have access to new avenues in discovering and recording high-quality data from sources such as speech, body language, and proxemics (Moura, 2009) that we can complement with 'ground truth' measures. Additionally, given the underdeveloped causal foundations of some social signals, this chapter offered the "abduction paradigm" as a sound theory-building alternative to stimulating new theoretical insights (Timmermans \& Tavory, 2012). Chapters 3 and 4 exemplify two end-to-end studies conducted with the sociometric badges following the steps previously described and employing machine learning based methods of analysis; both studies draw from the data-driven theory development paradigm, and contribute to social science by combining the work on linking sensor-based social signal data to behavior-based social phenomena. 
This chapter is based on Francu, E., van den Bossche, P., Segers, M., Lemmink, J. (2019). It Takes Two to Tango: Interaction Synchrony and Creativity in B2B New Service Development. Working Paper

An early version of this paper was presented at the European Marketing Academy (EMAC) $28^{\text {th }}$ Doctoral Colloquium, Marketing Strategy, Beginner Track, May 2015. 


\section{Chapter 3}

\section{It Takes Two to Tango}




\begin{abstract}
Involving customers as "co-developers" is recognized as a central pillar of innovation in the context of new $\mathrm{B}_{2} \mathrm{~B}$ service development. Such processes involve close human interaction and collaboration between a focal company and its end-user customers to clearly establish their needs as a basis for purpose-driven solution development. However, these collaborative practices inextricably involve information asymmetries, in terms of customer needs and wants, project goals, and social familiarity. In the innovation literature, these asymmetries are a potential source of innovation. However, said potential needs to be nurtured by effectively managing the interaction between the $\mathrm{B}_{2} \mathrm{~B}$ parties.

In this study, we address these information asymmetries by distilling human behavior down to a level of nonverbal (e.g. meta-verbal and body language) proxies of the emergence of creative outcomes in teams comprising both customers and firms. In this way, this study seeks to answer questions around the extent to which teams comprising members of normally somewhat disparate communities coagulate when presented with a common, mutually beneficial collaborative goal.

Specifically, this involves comparing MRQAP cause-effect models with state-of-the art supervised classification approaches, in the search for key variables that influence the coming together of group members in pursuit of creative outcomes. In addition to exploring co-development between firms and their customers, this study is unique in that in follows teams throughout a comprehensive breakdown of the ideation process. This comprises establishing a project roadmap, stakeholder assessment, customer persona development, customer journey development and meeting wrap-up sessions.

Results show varied effects for the capacity of individual nonverbal variables as predictors of creativity across meetings, with different body language variables demonstrating predictive power in earlier meetings, and conversational features demonstrating predictive power in the last meeting. However, in shifting to a discriminative prediction approach, results showed that both conversational and body language features represent strong proxies for creative outcome prediction, with turntaking and proximity alone contributing an average of $29.87 \%$ improvement to models, peaking at an average accuracy of $74.40 \%$ across meetings.
\end{abstract}

Keywords: New service development, customer involvement, social signals, sociometric badges, team creativity, MRQAP, multi-class classification 


\section{Introduction}

Sixty percent of Chief Marketing Officers (CMOs) worldwide assert that the traditional industry taxonomies are collapsing as companies create new sector hybrids by applying their knowledge to other sectors (IBM, 2016). To stay ahead of the competitive curve, torchbearer CMOs focus on understanding their customers and co-developing new products and services with them, while being set to renounce a certain amount of managerial control and shifting towards collaborative business models. Customer-collaboration is thus becoming more crucial to companies' business strategy due to the unique relationship they develop with their customers who are an essential pool of insights for the development of new products and services (Iyer \& Davenport, 2008). In this respect, company performance is highly dependent on how marketers and new product and service development teams engage their customers to co-create value and innovate for the future (IBM, 2016).

Whilst industry disruption and changing consumer needs are presently attributable to predominantly technological factors, many of the concepts that define marketing and new product and service development remain very 'human' (He, 2013). This especially includes the human-centric nature of collaboration and co-development, as well as the humancentricity embedded in the definition of innovation as a process of creating, refining, and implementing new ideas that create value for customers (Amabile, 1996; Linder, Jarvenpaa, \& Davenport, 2003; van de Ven, 1986). Thus, in addressing calls to develop a deeper and more comprehensive understanding of interaction between firms and their customers in innovation processes ( $\mathrm{Cui} \& \mathrm{Wu}, 2015$ ), there exists a conceptual necessity to address the gap of understanding natural human dynamics and behavioral processes. This gap comprises three main sub-challenges.

Firstly, involving customers in innovation processes does not come with a fixed protocol; there are several types of customer participation activities. Some scholars classify them across two dimensions: customer participation as an information resource (CPI) and customer participation as a co-developer (CPC) (Fang, 2008). More recent research depicts three categories of customer participation: as an information source (CIS), as co-developers (CIC), as innovators (CIN) (Cui \& Wu, 2015, 2017). Additionally, previous cross-sectional research based on primary data from multiple industries showed that it is difficult to translate customer participation into innovation success (Cui \& Wu, 2015). To address this challenge, this study focuses on uncovering the co-development process by quantifying the natural human dynamics and behavioral processes (i.e., the socio-behavioral structures) underpinning it.

Secondly, in thinking about a starting point for defining a possible customer involvement in innovation protocol, it should be noted that interactions between the firm and participating customers in new service development (NSD) involve an exchange of information when the previously existing knowledge is asymmetrical. It is crucial for firms and their customers to reach a common understanding and agreement during the ideation process (Bstieler \& Hemmert 2010), factoring in that customers often have relatively limited innovation experience and competence (Christensen, 2013; Christensen \& Bower, 1996; Verganti, 2008), and that customers are often unable to voice their latent needs in a clear manner (Franke, Keinz, \& Steger, 2009), particularly in $\mathrm{B}_{2} \mathrm{C}$ contexts. B2B contexts by contrast often involve 
actors able to appropriately understand an NSD project's goals and thus more easily communicate their needs (Mahr et al., 2014). This challenge can be addressed from the perspective of social physics (Comte, 1856; Pentland, 2015), specifically, with the understanding that a process analogous to the second law of thermodynamics dictates that when two social entities are allowed to interact, given enough time, information exchange forces will ultimately see the entities reach a mutual knowledge equilibrium (Adami, 2004; Adami \& Hintze, 2013). More specifically, this study addresses this challenge by investigating granular objective social interactions to quantify and assess the knowledge exchange and idea evolution.

Thirdly, at a more micro level, little is known about what 'local' (actor / dyad level) interactions can be deemed optimal in co-developing processes. For instance, seminal work into the creative nature of new product development highlights the importance of fast and rich feedback though face-to-face communication and how communication is inverselyproportional to spatial separation between members (Allen, 1977). Thus, higher physical proximity seems to increase the frequency of communication, which in turn fosters the performance of the development team (Pinto, Pinto, \& Prescott, 1993). Relatedly, the literature stream on communication accommodation suggests that dyadic communication patterns are affected by the amount of speech mimicry displayed by the interlocutors, with the communication distance the speakers create between each other is regulated by verbal and nonverbal aspects of communication such as lexis, tone of voice, speech style, etc. (Shepard, Giles, \& Le Poire, 2001). We address this challenge by generating the aforementioned structural and knowledge exchange proxy data on the basis of social signals, both verbal and nonverbal.

In sum, we aim at addressing this gap and its constituent sub-challenges by investigating the symbiosis between the firm and its customers that facilitates and shapes innovation, focusing on the co-development process. Drawing on the new service development (NSD), creativity, and team dynamics literature, we aim to show; (a) the usefulness of a socio-structural approach (observing objective social interactions) to disentangling the co-development process and working towards the definition of a co- development best-practice protocol, (b) the validity of socio-behavioral proxies as markers of knowledge exchange and idea evolution, and (c) revealing the factors driving the interplay that brings about highly creative interactions. We conduct a field study, involving primary data from a co-creative NSD project to investigate the interactions between $\mathrm{B}_{2} \mathrm{~B}$ actors involved in this process in the ideation phase, focusing participating customers as co-developers (Cui \& $\mathrm{Wu}, 2015$ ). We show the extent to which the face-to-face interactions bring about creativity in the development process, focusing on the business customer's high capacity to voice their latent needs.

Additionally, we address another gap in the literature concerning quantifying the team's natural human dynamics by employing socio-behavioral measurements (Kozlowski, 2015) in three ways. First, by looking at physical proximity as the main prerequisite to objective NSD process involvement as being around each other entails being exposed to the flow of information between the firm and customers. Second, we distinguish between proximity and actual face-to-face encounters between actors, and shed light on how differently they affect the creative outcome. Third, we investigate objective communication patterns (the 
communication segments or turns taken by the participants) as the channels through which ideas flow and are shaped. We circumvent the limitations of traditional data collection by employing an innovative tool to collect the second-by-second dyadic social signals given away by a NSD team and their participating customers (i.e., time spent face-to-face, time spent in proximity, number of speech turns taken, percentage of body movements mirroring). The objective data was collected with real-time measurement devices (i.e., sociometric badges - sensor devices that continuously measure the various non-linguistic aspects of interaction and the movement patterns based on the relative location and proximity of individuals).

\section{Literature review}

\subsection{Customer Involvement, Creativity, and Innovation}

The innovation potential stems from the diversity of ideas in the customer involvement process. As covered extensively in Chapter 1, the key promise of customer involvement is that it improves innovation performance by advancing the firm's knowledge on customers' needs for which to develop tailored solutions. The most recent taxonomy depicts three types of customer involvement: as an information source (CIS), as co-developers (CIC), as innovators (CIN) (Cui \& Wu, 2015, 2017). Each of the three entails a distinct strategy of managing customer knowledge. CIS requires limited behavioral involvement of customers and mostly absorbs their contribution through channels such as online fora; the communication and knowledge flow are mostly unidirectional to the company. CIC actively involves customers in the development process online as well as in face-to-face interactions; the customers are an essential part of the development team and the two entities are in constant dialog throughout the process. CIN requires no active involvement with the customer and the development is mostly outsourced to them.

Of the three, CIC describes the closest collaboration between firms and their customers in the NPD process. One prominent example of CIC are professional service providers (i.e., consultancy firms) that co-develop solutions for and with their $\mathrm{B} 2 \mathrm{~B}$ customers. This approach to involving customers takes a customer-centric innovation perspective (Sawhney, Verona, \& Prandelli, 2005).

The customer's role is active as they are active partners in the innovation process and de facto members of the development team. The direction of the interaction between the two entities is two-way and the back-and-forth dialogue with customers is continuous and of high intensity. The richness of the knowledge exchange is high and the focus of the dialogues is on social and experiential knowledge. The size and scope of the collaboration is on the benefits rendered for prospects and potential end-users (Sawhney et al., 2005).

Previous cross-sectional studies based on primary data from multiple industries revealed that it is difficult for companies to translate customer participation into innovation success (Cui $\& \mathrm{Wu}, 2015)$. It is not yet known how the co-development process evolves and what kind of interactions between the two spheres are beneficial to NSD.

This type of involvement requires very carefully balancing process interdependence between firm and customer. If process interdependence is low, involvement can improve speed to 
market but impede new product innovativeness; conversely, high interdependence weakens speed to market (Fang, 2008). To date the extant literature does not offer evidence regarding the optimal process interdependence level that positively affects the whole spectrum of NPD outcomes (Cui \& Wu, 2017).

The knowledge exchange requires a degree of novelty and usefulness of ideas (i.e., creativity) to advance the development of bespoke solutions. Depending on the type of task at hand, creativity literature offers three conceptualizations of novel creative outputs (1) verbal creativity or creative storytelling, (2) artistic creativity, and (3) creative problem solving (Amabile, 1996). Of all three, the latter best addresses innovation tasks. This literature defines this type of creativity as the generation of new and ideas worthwhile implementing (Amabile, 1996; van de Ven, 1986). When it comes to the source of creative ideas, seminal research argues that the individual creative potential is the catalyst of innovation (Bharadwaj \& Menon, 2000; Scott \& Bruce, 1994). More recently, greater emphasis is now placed on the dynamic aspect of social interactions and less on the factors that influence the individual's social cognition and its outcomes (Brennan \& Enns, 2015; De Jaegher et al., 2010; Heerey, 2015). This novel angle acknowledges the importance of the individuals' contribution but highlights that the individual creative ideas occur and are refined in the (social) interaction between team members (Perry-Smith \& Shalley, 2003).

With the rise of project-based collaboration within and across firms, team creativity has received an increasing amount of interest. This perspective argues that since the work pattern has shifted from cross-sectional collaborations to project-teamwork, it is crucial to elevate the level of research interest and analysis to team-level outcomes of the creative interaction. A growing body of literature highlights the social side of creativity. This revamped way of looking at team creativity acknowledges the importance of the individuals' contribution but emphasizes that the individual creative potential sparks in the (social) interaction between team members (Perry-Smith \& Shalley, 2003). There is still an essential gap in the literature on variation of the role of the social interactions throughout the stages of creative pursuits (generation, elaboration, and implementation) (Perry-Smith \& Shalley, 2014).

\subsection{Nonverbal Behavior and Creativity}

Returning to the fundamentals of customer involvement, the pivotal work of Allen (1977) into new product development as a creative process shows the importance face-to-face communication and its potential to nurture timely and rich feedback. He also shows physical proximity increases the frequency of communication. His findings are supported by later research that links the frequency of communication to the performance of the development team (Pinto et al., 1993). These seminal views involve the intersection of both verbal and nonverbal socio-behavioral processes.

When it comes to verbal behavioral processes, research conducted into linguistic style match covers holistically the influence of the content on the communication outcome. For instance, communication convergence has a positive effect on keenness, rapport building, and on team performance (Gonzales, Hancock, \& Pennebaker, 2010; Scissors, Gill, \& Gergle, 2008). On the other hand, seminal experimental research seems to suggest that some nonverbal aspects of communication (e.g., the synchrony of tones of voice over time) are conducive to better 
performance (Bernieri \& Rosenthal, 1991). Conversely, there is no general consensus in the literature on the direct creative outcomes of nonverbal aspects of communication, but there is theoretical precedent in the emotional cues embedded in nonverbal communication.

\subsubsection{Effects of Nonverbal Communication}

The fundamental study of decoding how a message is conveyed is also known as metacommunication or "communication about communication" (Mateus, 2017; Ruesch, Bateson, Pinsker, \& Combs, 2017). Some scholars consider this parallel communication channel consists of paralanguage (i.e., pitch, volume, pauses, and pace) and nonverbal communication (i.e., proxemics and kinesics - including gestures and facial expressions) (Mateus, 2017). Other scholars classify paralanguage under the umbrella-concept of nonverbal communication (Bernieri \& Rosenthal, 1991). In this study we will consider communication in its complexity and adhere to a holistic approach to understating its composing parts, namely proxemics (Hall, 1966), paralanguage (Trager, 1958, 1960, 1961), and kinesics (Birdwhistell, 1952) as socio-behavioral proxies for knowledge flow. We present an overview of fundamental findings in the homonymous sections below.

\section{Proxemics}

The term 'proxemics' was coined by Edward T. Hall who defined it as an observation-based theoretical construct describing the ways humans use of space as defined by extant cultural context (Hall, 1966). His 'Proxemic Framework' provides a holistic classification comprising four categories of distances, namely intimate (spanning the 1 to $46 \mathrm{~cm}$ radius), personal (covering the 46 to $122 \mathrm{~cm}$ radius), social (across the $122 \mathrm{~cm}$ to $3.7 \mathrm{~m}$ radius), and public (spanning the $3.7 \mathrm{~m}$ to $7.6 \mathrm{~m}$ or more radius) and their effects. The social distance optimal for work-related interactions is estimated to occur in the 1.2 to $2.1 \mathrm{~m}$ interval.

Other studies examined seating arrangements (Osmond, 1957; Sommer, 1967) to determine spatial configurations that bring people together and foster social interactions (i.e., sociofugal spaces) or, in contrast, discourage them altogether (i.e., sociopetal spaces). On the basis of Osmond's sociofugal - sociopetal spaces (1957), Hall (1963) develops a nine-point notation point for interpreting the angles at which individuals interact. The caveat of this code is that have been developed for the North-American population which is a notable mention since proxemics communication is always context specific and culturally defined. The eight angular orientation are coded on a circular scale from o (representing two individuals face-to-face) to eight (representing two individuals back-to-back); the rest of the seven positions are evenly distributed on the circular scale at $45^{\circ}$ increments. The most frequently observed are positons o, 1, 2, 4, and 8 where o (i.e., face-to-face) represents direct communication where the (mutual) intent is reaching the interlocutor with maximum intensity (idem).

Recent advances in the field of computer science lead to the development of ubiquitous computing technologies (e.g., wearable light tags) that collect angular and distance data; these devices have been successfully deployed to predict objective verbal behaviors as well as creative problem solving in design tasks using angular and distance data (where the data was coded using Hall's taxonomy) (Montanari et al., 2018) as well as how spaces influence 
communication in innovation (Brown, Efstratiou, Leontiadis, Quercia, Mascolo, Scott, \& Key, 2014).

\section{Paralanguage}

The study of paralanguage covers the characteristics of speech that nuance it and allow the speaker to convey meaning beyond words. These features include vocal cues (e.g., pitch range and control), rhythm control, and tempo (Burgoon, Guerrero, \& Floyd, 2016; Trager, 1961). Of the three, the vocal cues and their effects have been substantially studied.

In the literature stream of fundamental social psychology there is abundant evidence that nonverbal vocal cues convey a wide range of information about the speaker. It has long been shown that receivers can determine sender's age (e.g., Smith, 1980), gender (e.g., Helfrich, 1979), personality (Scherer \& Scherer, 1981), socio-economic background (Robinson, 1979), ethnicity (e.g., Giles, 1979), status in small groups (e.g., Scherer, 1979b), and affective states (Scherer, 1989) when exposed to a few sentences or even just single words or nonsense syllables spoken with different inflections (Kappas, Hess, \& Scherer, 1991). Most notably, the correct decoding of states from voice samples has been shown to be as accurate as or more accurate than the recognition of affect from facial expressions (Scherer, 1981).

With the exception of research conducted on affective states, the major limitation of these studies resides in their design; more specifically, the used stimuli (e.g., isolated words, nonsense syllables, numbers, etc.) may prompt speakers to adopt stereotypical interpretations of the task leading to judges' overestimated accuracy of the judgement. Thus, these judgement studies lack ecological validity which, unfortunately, makes the interpretation and the further application of these results problematic (Kappas et al., 1991).

Given the robust body of literature stating the importance of affective states emitted through vocal indicators dating back to Darwin the topic has been enriched by decades of systematic studies on emotional reactions (e.g., Chronaki, Wigelsworth, Pell, \& Kotz, 2018; Darwin \& Prodger, 1998; Wallbott \& Scherer, 1986).

Let us continue this synopsis by introducing the simplified distinction of the three levels of analysis of vocal communication and the relationship between them. Firstly, at procedural level, the sender produces a vocal utterance using their voice production system (Scherer, 1989); secondly, at acoustical level, the utterance thus produced travels though air carrying the objective representation of the voice signal (e.g., fundamental frequency, amplitude ratio, volume, etc.); lastly, at perceptual level, the receiver decodes the voice signal (e.g., loudness, pitch, register, etc.) (Shannon \& Weaver, 1963).

Later conceptualizations refer to the first level as an externalization of the sender's state conveyed through two channels, namely verbal (i.e., content) and meta-verbal (i.e., the specifications of the voice signal), to a receiver to decode (Scherer, 1978). The speaker's state, revealed by changes in their vocalization, has been shown to be influenced by muscle tremor, breathing rate, and dryness of the mouth and larynx (Ohala, 1981). In other words, one's emotional state induces bodily changes that affect speech production that can be decoded 
by an interlocutor. The different combinations of variation in acoustic parameters ${ }^{6}$ (i.e., fundamental frequency or $\mathrm{F}_{\mathrm{o}}$, intensity, frequency range, high-frequency energy, intonation, speech rate, etc.) describe distinct voice types associated with certain emotional states. For instance, boredom-indifference is an indication of decrease in $\mathrm{F}_{\mathrm{o}}$ and mean intensity; irritation-cold anger is identified by an increase in $F_{0}$, high-frequency energy, and downwards intonation; rage-hot anger is defined by an increase in $\mathrm{F}_{\mathrm{o}}$ and speech rate and a mean intensity; of joy/elation is characterized by an increase in $\mathrm{F}_{\mathrm{o}}$ and a mean intensity and $F_{o}$ and speech rate variability over time (Banse \& Scherer, 1996; Kappas et al., 1991; Scherer, 1979a).

As previously introduced, these findings stem from fundamental studies in social psychology. These outcomes have been taken on in the past decade by the field of computer science in an effort to quantify and better analyze paralanguage. The new field of computation of social science is augmenting our previous understanding of influence of changes in pitch, loudness, rate, and tone on expressive prosody (e.g., Ponsot, Burred, Belin, \& Aucouturier, 2018) and on emotions (e.g., Sun, Moore, \& Torres, 2009). These novel and computationally fit studies are limited to experimental settings with low numbers of participants, thus limiting the results' ecological validity.

Beyond absolute sound metrics of prosodic features, paralanguage includes the evaluation of speeches' interruptions (Mateus, 2017; Trager, 1961). Most languages studied are predominantly composed of small units of speech occurring in swift exchanges. In conversation analysis, these units are known as utterances (i.e., a unit of speech delimited by clear pauses) or 'conversational turns'; the act of exchanging turns is referred to as 'turntaking'.

There is no agreement as to how to set the length boundaries of an utterance unit (Oller, 1973; Traum \& Heeman 1996), but these speech acts are, in average, 2 seconds long with interruptions around 200 milliseconds across languages and are proxies for grammatically complete phrases or clauses (Levinson, 2016). Turn-taking has been shown to be 'the interaction engine', one of the evolutionary artefacts of human communication that facilitates face-to-face interactions and the use of gestures (Enfield \& Levinson, 2006; Levinson, 2006).

\section{Kinesics}

The term 'kinesics' refers to the study of visible body movements such as facial expression, gestures, posture, and gait (Birdwhistell, 1952). In the field of social psychology, the earliest fundamental study on posture was conducted by James (1932) whereby he reveals how subjects' postural orientations communicate feelings or attitudes. Broadly, his findings suggest that leaning forward communicates relatively positive attitudes whereas turning away or leaning backwards conveys negative attitudes. Specifically, he describes four archetypes of postural layouts and their respective message conveyed. Firstly, the 'approach' type is described by a forward leaning body that communicates attention. Secondly, by

\footnotetext{
${ }^{6}$ These vocalization variations are of particular relevance for Chapter 4 in support of the vocalics variables.
} 
turning away or 'withdrawing' a person communicates repulsion. Thirdly, 'expansion' is described by expanded chest, upright or backward-leaning trunk, straight head, and raised shoulders; this archetype communicates superiority or disdain and is relevant in assessing status differences. Lastly, 'contraction' is defined by a sunken chest, a forward-leaning trunk, a bowed head, and wilting shoulders; this archetype is indicative of a depressed or downcast individual and needs to be considered in and of itself as it does not communicate their attitudes toward a receiver.

Studies that have shortly followed have shown a relation between postural styles and personality characteristics (Allport, 1937; Lowen, 1958), motivations, attitudes, and intentions that may not be spoken (Deutsch, 1947, 1952; Fromm-Reichmann, 1950)

From an anthropological stand-point, it was hypothesized that body movements are patterned by culturally specific interactions and cannot be assessed as universal (Birdwhistell, 1952; Kendon \& Sigman, 1996).

\section{Combined Effects and Behavioral Synchrony}

These distinct behaviors do not occur in isolation or in a regular sequential pattern. It has been shown that different combinations of nonverbal behaviors have specific sources and/ or render explicit meaning.

Some experimental studies (Rimé \& Gaussin, 1982; Rimé \& Schiaratura, 1991) have investigated the link between speech and body movements; the findings reveal that merely pronouncing words in a short-term memory task significantly increases the amount of body movement. Other studies have alternately restricted the movements of subjects' upper body during a 50-minutes conversation tasks; the findings revealed that the imagery's vividness significantly decreased in the restriction phases decreased compared to the free-movement baseline (Rimé, Schiaratura, Hupet, \& Ghysselinckx, 1984). According to the cognitive-motor view, the explanation for the inextricable link between speech and body movement is that our cognitive processes are embodied (Feldman, Feldman, \& Rimé, 1991). In simpler terms, our body movement is a behavioral manifestation of the act of thinking.

Nonverbal behavior has also been shown to function as a complementary feature to speech content. Some studies describe how, in conversations, postural changes are used as turntaking signals (Deutsch, 1947, 1952); for instance, speakers punctuate their turns by backward leans simultaneously with completing an utterance (Wiemann \& Knapp, 1975). Similarly, body movements have also been shown to serve as punctuation in dialogues (Feldman et al., 1991; Kendon, 1980).

The combined effects of nonverbal behaviors are not as elucidating as the results of the fundamental single-construct studies. An added degree of complexity is given by investigating how these behaviors occur over time. Some scholars argue that postural mimicry occurs over time while in another's proximity; it subconsciously communicates 'liking' and 'understanding' and fosters smoother social interactions and rapport building (Chartrand \& Bargh, 1999). Other scholars explain behavioral synchrony as general behavioral engagement, homophily, or consensus (Bernieri \& Rosenthal, 1991; Rico, SánchezManzanares, Gil, \& Gibson, 2008). 


\subsubsection{Antecedents of Creativity}

The informational theories of emotion explain that we use emotions as signals about the type of environment we are in, but that they also help us adapt to said environments (Fong, 2006). When it comes to the emotions - creativity link, some scholars argue that negative moods lead to higher creativity (George \& Zhou, 2002) but a broader body of literature suggests that positive emotions have beneficial effects on creativity (Isen, Daubman, \& Nowicki, 1987; Fredrickson, 2001). Explicitly, in the case of creative ideation (entailing fluidity, originality, and flexibility) the data show that positive moods enhance creativity (Davis, 2009).

Given the above, there are still gaps in the literature concerning empirical evidence to confirm the direct effects of proximity and face-to-face interaction and nonverbal communication on the creative process. Moreover, previous research relied solely on selfassessments, observations, and audio-video coding, tools that are known for their limitations and biases (Montanari et al., 2018; Onnela, Waber, Pentland, Schnorf, \& Lazer, 2014).

\subsection{Research Questions and Research Aim}

As presented in the introduction, the aims of this chapter are to develop a deeper and more comprehensive understanding of interaction between firms and their customers (Cui \& $\mathrm{Wu}$, 2015), by examining the natural human dynamics and behavioral processes occurring in the innovation process (Kozlowski, 2015). These three challenges give rise to the following research questions.

(a) The usefulness of a socio-structural approach

We adhere to the school of thought supporting customer involvement as facilitator of innovation. Customer's knowledge sharing is crucial as it brings in new project relevant input to the development process. Employing the co-developing method entails closely working with them as temporary members of the NSD project team. The project's output is highly dependent on the interaction and knowledge flow between team members globally, as well as in dyads. Thus, by investigating the objective behavior of NSD project teams we are closer to disentangling the co-development process and working towards the definition of a codevelopment best-practice protocol

(b) The validity of socio-behavioral proxies as markers of knowledge exchange and idea evolution

This chapter aims to shed light on the NSD project team dynamic by looking at the team's communication channels and nonverbal cues (i.e., social signals) (Pentland, 2008; PerrySmith \& Shalley, 2014; Roudposhti et al., 2016) as well as the communication content (creativity) (Amabile, 1988). The purpose observing the objective behavior of NSD project team members is to measure, quantify, and assess the prediction power said nonverbal interactions (i.e., social signals) has on the verbal behavior (i.e., creativity).

(c) Revealing the factors driving the interplay that brings about highly creative interactions.

By implementing (b) will uncover salient social signals and explain and their respective impact on team creativity over the ideation phase of a NSD project. 
Given the above, the objective of this chapter is to answer the following research question:

RQ: How do team social signals affect team creativity of a new service development project team?

In light of Chapter 2 and of the literature review on proxemics, paralanguage, and kinesics presented in this chapter, there are four explorative questions arising from RQ.

(1) Is physical proximity the main prerequisite to knowledge exchange? Our assumption is that being around each other entails being exposed to the flow of knowledge being exchanged between the firm and customers. We thus expect proximity to be the sine-quanon condition for high creative problem solving.

(2) Does the time spent in physical proximity affect the creative outcome differently than face-to-face time? We expect face-to-face time to affect creative problem solving in a different manner as such interactions are, by nature, closer and mostly observed in focused, one-on-one discussions.

(3) Could turn-taking be the best predictor of high creative problem solving? Given the literature explored on paralanguage, we assume that the communication patterns (the communication segments or turns taken by the participants) are the channels through which ideas flow and are shaped; turn-taking thus is a proxy for knowledge exchange and we expect a proportional effect on creative problem solving (i.e., the more turns taken, the more creativity experienced).

(4) Is the time spent in synchronously positive body movements facilitating team creative problem solving? On the one hand, forward leaning movements communicate relatively positive attitudes and attention (e.g., James, 1932). On the other hand, the cognitive-motor view posits that our cognitive processes are embodied (Feldman, Feldman, \& Rimé, 1991) and body movements are behavioral manifestation of the act of thinking. From the two we can infer that similar forward leaning movements of team members interacting is a proxy for synchronous thinking that facilitates knowledge exchange.

As introduced in Chapter 1, we propose signaling theory (ST) (Connelly et al., 2011) as a meaningful theoretical lens for explaining the behavioral collaboration patterns in the case of information asymmetry (Kao \& Schmandt, 2015; Liu \& Donath, 2006). We make the parallel between the notions of macro-level signaling as described by ST (Connelly et al., 2011) and the micro-level signaling where the external signals are used as input for adjusting the response to social interactions. In the case of both levels, collaborating entities start from a state of information asymmetry and need to adjust the interplay of interactions to uncover latent knowledge (Connelly et al., 2011), needs, and ideas to best develop a new offering.

Through a multi-methodological approach that coupled sensor based analysis, wearable computing, and creative behavior assessment this investigation helps us better understand the nature and mechanisms of team creativity in the wild. We show the extent to which the nonverbal behavior of team members leads to creativity in the innovation process; we overcome the limitation of customers' limited capacity to voice their latent needs by looking at business customers. It was previously introduced that the business customers are thought to have more appropriate knowledge about the NSD project's goals and about their needs 
(Mahr et al., 2014). The setting is highly relevant for mangers as the $\mathrm{B}_{2} \mathrm{~B}$ market is growing (as discussed in Chapter 1).

\section{Method}

\subsection{Participants and Setting}

Our sample consisted of a NSD project team involving representatives of the two companies -a large recruitment service provider and a service innovation consultancy agency. For the purpose of this project, the agency delegated four employees while the client delegated ten. The joint NSD team consisted of 14 team members. The principal investigator for this project acted as an observer during the three meetings. The aim of the project was to innovate the offering of the client by radically improving the way the end users are reached. The agency planned the project in what they call "the heart-beat project process" as depicted in Figure 1 below.

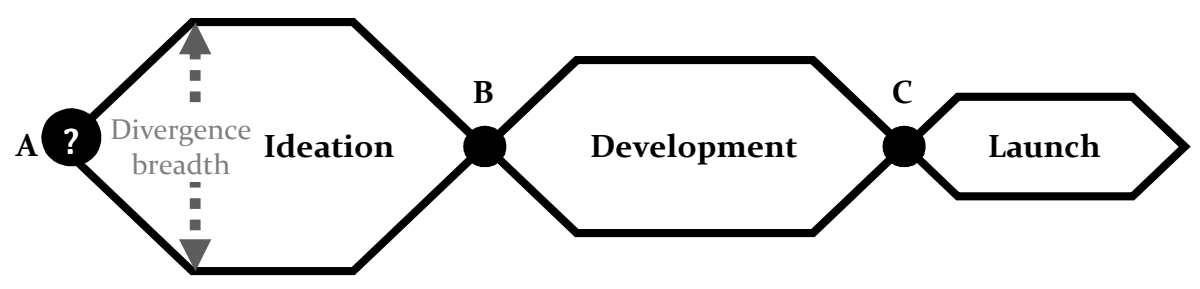

Figure 1. The "heart-beat" NSD project process

The three project phases are in line with the very recent taxonomy of Chang and Taylor (2016), namely (1) ideation (understanding the client's current offering and end-users and designing and incipient prototype - A to B), (2) development (creating and refining the final prototype - B to C), and (3) launch (activation of the final prototype - C to D).

Following definition of innovativeness by Sethi, Smith, and Park (2001) one must presume a degree of creativity in the ideation and design processes. To answer our research questions, the present field study focuses on the ideation phase (the first three months - A to B) which consisted of three joint meetings. The goal of these meetings was (a) to gain a clear overview of the client's current service offering, (b) better understanding their end-users, and (c) designing the foundation of the new service prototype, respectively.

\subsection{Meetings' Format}

The agency designed the NSD project process in collaboration with the firm. The agency managed the project process and designed the setup of the three meetings, planned the tasks and assigned the respective design tools to each task. The total duration of this project phase was three months. The meetings were one week, respectively two months apart.

As the principal investigator of the study acted as an unobtrusive observer during the three meetings, we could label each meeting by the conversations' content. In each of the meetings, the employed service design tools aimed at guiding the discussions for the completion of the tasks. When the members' communication was bounded by the tasks tacked we labeled the meeting as task-oriented. When the communication focused on 
involving all members into negotiating the best possible ideas for the service prototype we labeled the meeting as people-oriented.

The observations also allowed us access to the structure of each meeting dictated by the service design tools used. We thus obtained a split of the meetings in six, twelve, and eleven composing episodes respectively. Table 1 depicts the episodes per meeting.

Table 1. The detailed description of the episodes per meeting

\begin{tabular}{|c|c|c|}
\hline $\begin{array}{l}\text { Meeting } 1 \text { - } \\
\text { Exploration and } \\
\text { ideation }\end{array}$ & $\begin{array}{l}\text { Meeting 2 - } \\
\text { Prototyping } \\
\text { the idea }\end{array}$ & $\begin{array}{l}\text { Meeting 3- } \\
\text { Pitch of } \\
\text { final prototype }\end{array}$ \\
\hline $\begin{array}{l}\text { 1. [10 min] Meeting } \\
\text { intro: project aim } \\
\text { and roadmap } \\
\text { 2. [30 min] Assessing } \\
\text { existing stakeholders } \\
\text { 3. [50 min] Crafting } \\
\text { personae \& } \\
\text { stakeholder map } \\
\text { (short intro \& sub- } \\
\text { team work) } \\
\text { 4. [33 min] Sub-team } \\
\text { personae } \\
\text { presentations } \\
\text { 5. [50 min] Crafting } \\
\text { current customer } \\
\text { journeys } \\
6 . \text { [14 min] Outro \& } \\
\text { wrap-up }\end{array}$ & 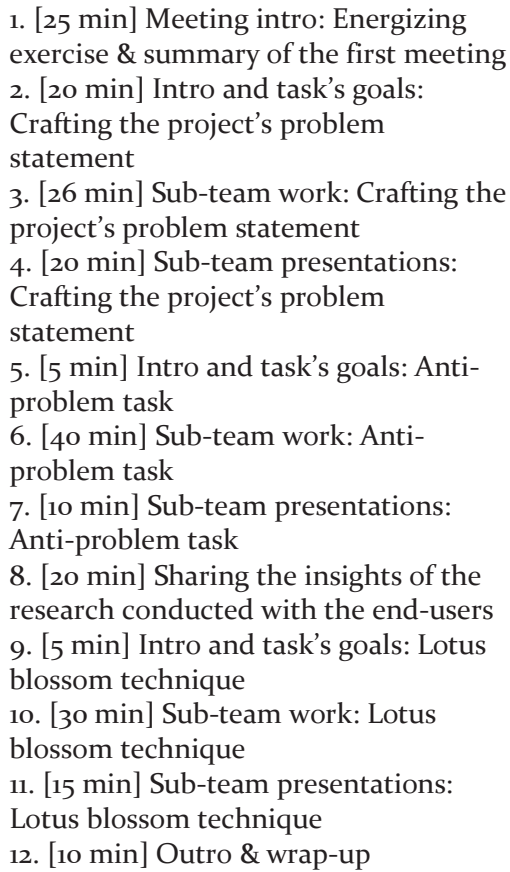 & $\begin{array}{l}\text { 1. [40 min] Setup and preparation } \\
\text { of tools } \\
\text { 2. [10 min] Intro: pitching the } \\
\text { two possible prototypes - the } \\
\text { incremental and the radical new } \\
\text { services co-designed } \\
\text { 3. [5 min] Negotiating the pursuit } \\
\text { of the radically innovative } \\
\text { concept } \\
\text { 4. [10 min] Pitching the radically } \\
\text { innovative prototype } \\
\text { 5. [12 min] Firm's input in the } \\
\text { prototype } \\
6 . \text { [ } 15 \text { min] Implementation } \\
\text { roadmap } \\
7 . \text { [ } 47 \text { min] Business model } \\
\text { canvas } \\
8 . \text { [ } 20 \text { min] Presenting the new } \\
\text { service prototype } \\
9 . \text { [ } 12 \text { min] Hypotheses testing } \\
\text { and implementation process } \\
\text { 10. [15 min] Further avenues to } \\
\text { pursue in the next phase: API } \\
\text { economy } \\
\text { 11. [ } 20 \text { min] Outro \& wrap-up }\end{array}$ \\
\hline 187 minutes & 226 minutes & 206 minutes \\
\hline
\end{tabular}

In the first meeting, content-wise, the agency introduced the project aim to the joint project team, explored the status-quo of the business model, and tied to get a better understanding of the end-users. From a team dynamics perspective, the observed focus of the meeting was on opening the communication channels amongst the member of the newly formed team to facilitate the emergence and sharing of latent knowledge. As depicted in the first column, the meeting had six composing episodes for each topic task tackled. During the second meeting, from a content standpoint, the team explored the valuable ideas generated in meeting one; the agency representatives informed the joint project team of the results of the research the agency representatives conducted on the client's end-users, and together they designed the first service prototype. As for the team dynamics, the observed focus of the 
meeting was on exploring and building upon the ideas shared in and as of meeting one. The team was randomly split in three sub-teams for the completion of tasks two, three, and five respectively. Due to the intensive sub-team work, we can assess the meeting as "task focused". As shown in the second column, the meeting had twelve composing episodes for each task undertaken.

The third meeting consisted of the agency's pitch (to the joint project team) of the final service prototype. As illustrated in the third column, the meeting had eleven composing episodes for each topic deliberated. The now fully formed project team purposefully adopted a negotiation behavior meant to decide on the final new service prototype to be further tested and developed. In other words, from a team dynamics point of view, the observed focus of the meeting was both task- and people-focused.

Figure 2 depicts the three meetings as depicted by the agency. The first two meetings established the foundation of the joint team allowing its members to create a psychological safe environment, to diverge in the ideation process, to explore the possible avenues, but also to decide on ideas that are useful to take on in the converging phase. The third and final meeting concluded with the positive outlook on the new service prototype to be developed and tested in the upcoming project phases.

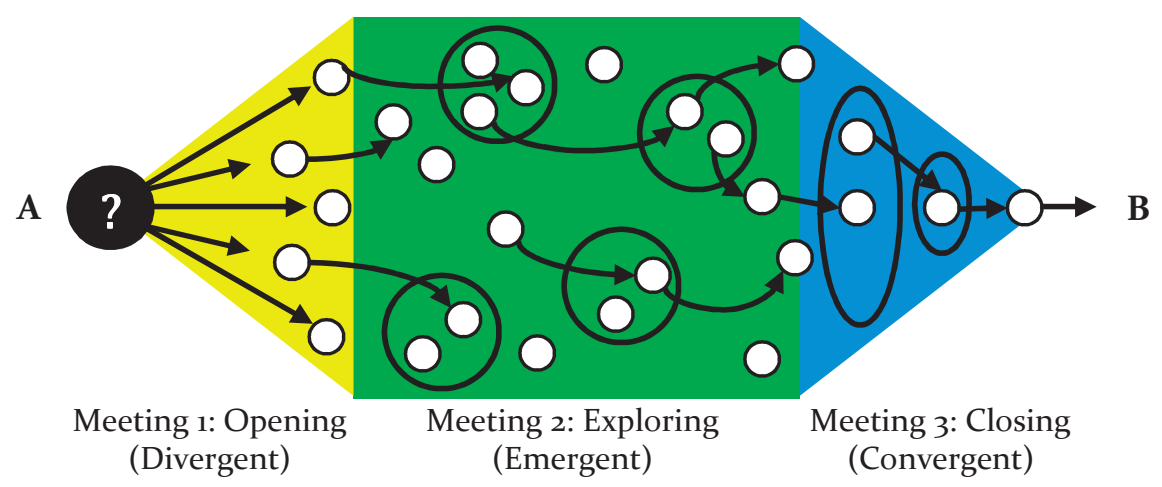

Figure 2. The ideation phase of the project process - the three consisting meetings (where A and B are corresponding to Figure 1.); the bullets are representative of the ideas' flow throughout the meetings.

\subsection{Procedure}

We kicked-off the first meeting with a presentation of the current study in which we informed the team members about the purpose, mechanics, and required involvement. We then handed out consent forms. After receiving their formal signed consent, we proceeded to handing out the sociometers. Immediately before the start of the next two meetings, we unobtrusively handed out the badges. At the end of each meeting, we collected the devices, 
sent out the creativity assessing survey (with a five days' deadline). We used the same approach for every one of the three project meetings.

\subsection{Data Collection and Variables}

The social signals that make up the constructs we study are based on the signals collected
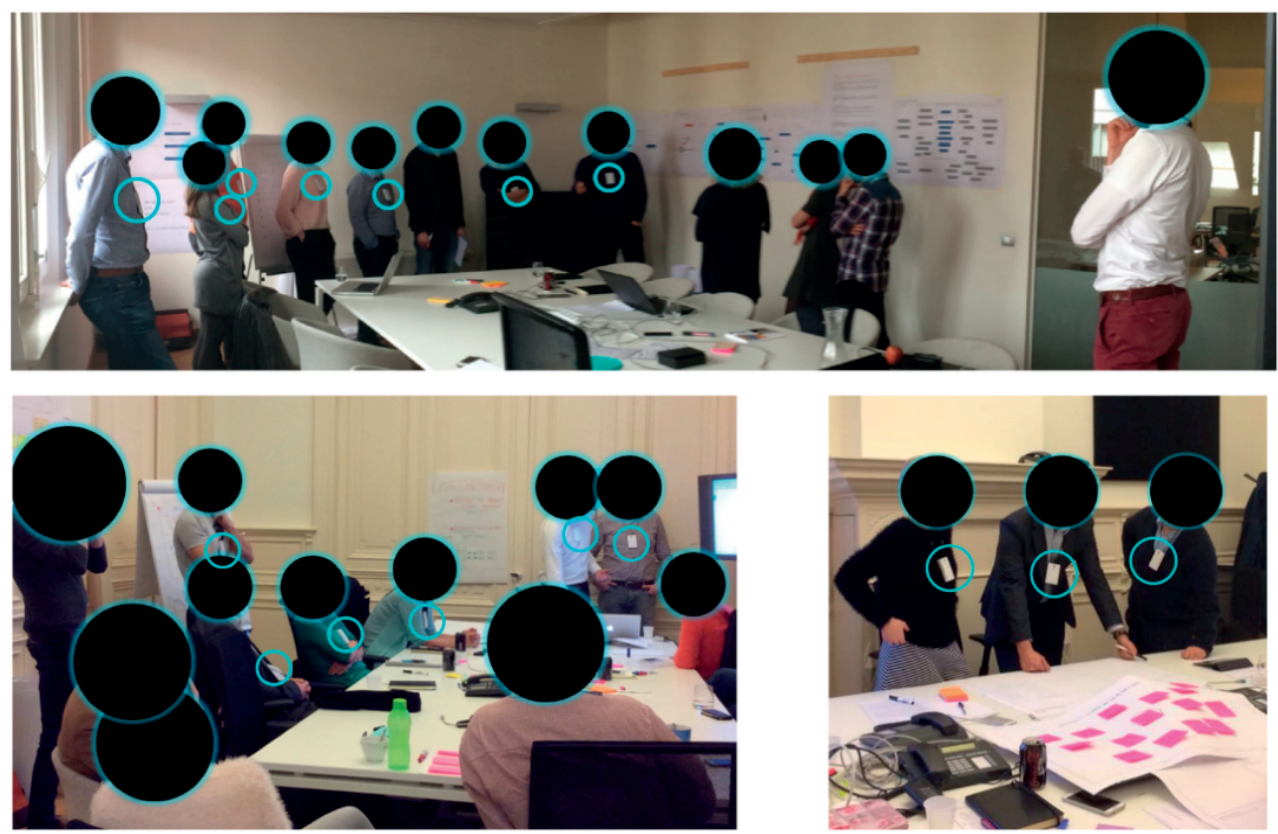

Picture 1. Participants wearing the badges during project meetings

with sociometric badges (or sociometers) (Kim et al, 2012; Pentland, 2008). The sociometers are "environmentally aware" wearable computing devices (Tripathi \& Burleson, 2011) that allow to capture badge-wearer's "social signals" derived from the presence relative to others, speech features, and body movement (Pentland, 2008). Several studies have extensively validated the badges (Basu, 2002; Choudhary, 2004; Kim et al., 2012; Olguín-Olguín et al., 2009; Pentland, 2008; Tripathi \& Burleson, 2011). (i) The badges scan Bluetooth signals emitted by the other sociometers every 5 seconds thus we have a proxy for the time they spent in each other's proximity [PRX]; (ii) the badges scan for other badges' infrared signals (IR) thus we have a proxy for the spent face-to-face $\left[\mathrm{F}_{2} \mathrm{~F}\right]$; (iii) the badges are able to quantify the user's overall speaking/ silent time, we obtain the structure of the team members' communication channels made up by the speech turns each of them take, or "turn-taking" [TTK]; (iv) the badges can measure the user's overall body movement energy in units of "gravity" (force per unit mass over time) we can thus extract the relative amount of time they spent in positive body movement (or energy) mirroring [BMM] and respectively in posture mirroring $[\mathrm{PM}]$. The badges did not record speech or conversational content.

As introduced, participants' creativity was evaluated over time by administering a short survey at the end of every meeting. As part of a larger assessment of creativity, the questions 
aimed at firstly validating if the participants experienced any creative moments; we insured the uniformity of the frame of reference by providing our respondents with the mostly agreed upon definition of creativity phrased as "literature defines creativity as the generation of novel and useful ideas" (Amabile, 1996). Secondly, we tasked the respondents with evaluating on a scale from one to 100 their individual creativity level and the creativity level of every other team member, as they perceived it during the project meeting.

This procedure strongly abides by the previously set standard for contextualizing and studying "creative problem-solving" (Amabile, 1983, 1996). It is for this purpose, that we do not include any specific tests of creativity but rather investigate subjective creativity as verbal behavior that is a consequence of contextualized social interaction. We linked the sociometric data to the creativity assessment using an anonymous identifier (i.e., the badge number).

\subsection{Data Manipulation and Variables' Operationalization}

The team members are connected in a social network structure by five social signals that manifest simultaneously (i.e., PRX, F2F, TTK, BMM, and PM) and that lead to a creative outcome. In other words, the members are connected by five types of ties at the same time. Represented collectively, this type of complex pattern of ties is called multiplex (Hanneman \& Riddle, 2005; Portes, 1998). This type of data is stored as node-by-node matrices that are "stacked" as "layers" (or "slices") in a single file. We thus used a stack of layered social signals for every meeting, each layer corresponding to an episode (i.e., an unfolded multiplex network per variable per meeting).

As described previously, the tasks dictated the structure of each meeting (Table 1. The detailed description of the episodes per meeting). For meeting one, we obtained six layers for meeting two, we logged 12 layers, and for meeting three we found 11 layers. We used this split as the analysis window-width of each social signal measured; the creativity survey rendered a single-level outcome measurement per meeting.

There are three main reasons for using unfolded multiplex networks per variable per meeting. First, the sociometers collected data continuously during the meetings that were approximately 4 hours each including breaks; the side conversation during the breaks are not relevant to the purpose of our study and induce noise in the data. Therefore, for the higher accuracy of the sociometric data we exported each social signal at the time limit of each episode (Sociometric Solutions, 2013). Second, it is the methodologically accurate of storing data about multiple types of relations between actors in a single multi-valued matrix (Hanneman \& Riddle, 2005; Kapucu \& Hu, 2016). Third, our networks are relatively small in size and we can render graphs to show the unions and intersections of the multiple kinds of relations, and "animate" the changes in network structure over time (Hanneman \& Riddle, 2005). Appendix 2 exemplifies such an unfolded multiplex matrix.

Thus the resulting three datasets each comprise multiplex matrices based on dyadic measurement of the social signal variables (PRX, F2F, TTK, BMM, and PM) with 6, 12, and 11 layers respectively, and a simplex matrix representing mutual, dyadic evaluations of creativity. Below we describe in detail the variables' operationalization. 


\subsubsection{Time Spent in Proximity [PRX]}

Proximity serves as a necessary condition for social interaction, information exchange and the subsequent evolution of the creative process. We measure proximity using the number of Bluetooth pings that each badge recorded constructed the proximity adjacency matrices [PRX]. The actual detections happen at an interval of +- 5 s. Cells in the resulting adjacency matrix represent the number of Bluetooth pings recorded at one second resolution for each badge with all other badges.

\subsubsection{Time Spent Face-To-Face $\left[\mathrm{F}_{2} \mathrm{~F}\right]$}

As with proximity, we measure $\mathrm{F}_{2} \mathrm{~F}$ time with the number of infrared pings logged by each badge constructed the adjacency matrix for the face-to-face data $\left[\mathrm{F}_{2} \mathrm{~F}\right]$. The actual detections happen at an interval of +- o.1 seconds when the badges are directly facing each other (IR signal cone of height $\leq 1$ meter and radius $\mathrm{r} \leq \mathrm{h} \tan \theta$ where $\theta= \pm 15^{\circ}$ within 1 meter and 30 degrees). Cells in the resulting adjacency matrix represent the number of infrared pings recorded at one second resolution for each badge received by other badges.

\subsubsection{Turn-taking [TTK]}

We capture the flow of knowledge sharing by looking at the structure of the team members' communication channels. Talking is a necessary condition for sharing information thus making it a suitable candidate for a proxy of knowledge sharing. From the speech detection features, we extracted the speech segments of every team member; a speech segment is defined and computed as any continuous, uninterrupted length of speech longer than 0.5 seconds but shorter than 10 seconds made by a single person (Sociometric Solutions, 2013a). A speaking segment that occurs within 10 seconds or longer of another speaking segment defines as a "turn".

We capture the communication channels by exporting the turns taken aggregated at network level [TTK]. Figure 3 below depicts the adjacency matrix obtained by computing the number of turns recorded or, in other words, the number of times each badge spoke after each of all other badges.

\begin{tabular}{|c|c|c|c|}
\hline & Badge A & Badge B & Badge C \\
\hline Badge A & o & $\begin{array}{c}\text { Number of times Badge A } \\
\text { spoke after Badge B }\end{array}$ & $\begin{array}{l}\text { Number of times Badge } A \\
\text { spoke after Badge } C\end{array}$ \\
\hline Badge B & $\begin{array}{c}\text { Number of times Badge B } \\
\text { spoke after Badge A }\end{array}$ & 0 & $\begin{array}{l}\text { Number of times Badge B } \\
\text { spoke after Badge C }\end{array}$ \\
\hline Badge C & $\begin{array}{c}\text { Number of times Badge } C \\
\text { spoke after Badge A }\end{array}$ & $\begin{array}{c}\text { Number of times Badge } C \\
\text { spoke after Badge } B\end{array}$ & $\mathrm{o}$ \\
\hline
\end{tabular}

Figure 3. Description of the adjacency matrix representing the number of turns recorded for each badge in exchange with all other badges aggregated at a certain timeframe.

\subsubsection{Relative Time Spent in Positive Body Movement (Or Energy) Mirroring [BMM]} and in Positive Posture Mirroring [PM]

The movement mirroring features (i.e., BMM and PM) are derived from the accelerometer data and capture mutual animation and body language reactivity. The badges measured the 
team members' overall body movement energy in units of "gravity" (force per unit mass). The accelerometer data and posture estimation renders the energy magnitude over the three axes of measurement (front/back vs. right/left angles). The features we derived from the accelerometer data are the body movement (or energy) mirroring [BMM] and posture mirroring [PM]. We exported mirroring (both body movement and posture) for the secondby-second data collected for each pair's activity levels and postures respectively with a maximum lag of seven seconds (Pentland, 2008). The values at each timestamp range from zero to one, where zero indicates no similarity and one indicates the two data sequences are identical. We then calculated the time each pair spent in positive BMM and PM relative to the total time of interaction. The percentages of mirroring taking place between each back pair's activity levels makeup the BMM adjacency matrices. We computed the same operations for each back pair's postures, thus obtaining the PM adjacency matrices.

\subsubsection{Creativity}

We collected the "creativity" data by asking participants after every meeting to evaluate the level of creativity of each team member on a scale from 1 to 10o. Each participant had to reflect upon the content of the conversations occurred during each meeting; subsequently they were asked to evaluate to what extent each other participant had creative input that was valuable for the better outcome of each meeting. We defined and computed the creativity tie as the average of the alter creativity scores. We calculated the creativity adjacency matrices by the averaging the creativity scores each pair have given each other.

\section{Analysis}

The final sample consisted of 145 matrices giving rise to 20710 possible dyads extracted from the constituent meetings' episodes (i.e., six in $\mathrm{M}_{1}$, twelve in $\mathrm{M}_{2}$, and eleven in $\mathrm{M}_{3}$ ), across the five social signals (i.e., PRX, F2F, TTK, PM, and BMM) collected from the participant team members in each meeting (i.e., thirteen in $\mathrm{M}_{1}$, thirteen in $\mathrm{M}_{2}$, and ten in $\mathrm{M}_{3}$ ).

The analysis comprised three main steps that are detailed as follows. First, we computed network density measures to provide a global topological overview of the networks. To assess global network structural similarities and differences across behavioral networks we calculated the network densities for each variable in the three separate meetings (Hanneman \& Riddle, 2005). Each matrix in our dataset represents a different type of network tie (e.g., the different social signals) and each layer shows the same network tie at different points in time (i.e., longitudinal overview of the multiplex networks). Second, we conducted multiple regressions with quadratic assignment procedure (MRQAP) to analyze the global relationships between social signals and creativity. We conducted multiple regressions with quadratic assignment procedure (MRQAP) to analyze the relationships between social signals and creativity. Quadratic assignment procedure (QAP) is an inferential statistical procedure that randomly permutes identical matrices of the same set of network actors and calculates their standard error to analyze the level of association between them (Hanneman \& Riddle, 2005; Kapucu \& Hu, 2016; Krackhardt, 1988). As our data is relational, QAP is the appropriate method, as it does not assume independence of observations. Complementary to MRQAP, we calculated the density measures to provide topological evidence for network relationships. Third, we conducted a predictive analysis using the dyadic social signals as 
input to classify the different creativity levels with forward feature selection and multi-class classification to further uncover the specific signals conducive to creativity.

\section{Results}

\subsection{Descriptives for Global Network Measures}

We calculated and mapped the network densities for each variable in the three separate meetings to gage structural similarities and differences between the different social signal networks (Hanneman \& Riddle, 2005) based on directed, weighted networks generated from the sociometric variables. Topological density refers to networks characterized by high levels of interconnectivity as opposed to networks characterized by sparse edge distributions. Density measures are used to assess the level of connectivity and linkage among individuals operating within a network (Scott, 1988, 2017). In other words, the higher the density of a network is, the higher the information exchanges. In contrast, a low density depicts a sparse network that contains isolates and restricts communication, information, and resource flow within the whole network (Scott, 1988, 2017). Generally, for binary undirected data, density is calculated by summing the number of all valued edges (disregarding reflexive ties) divided by the number of possible ties. In this case, density is the average strength of ties across existing edges; in other words, every point marked in the figure below is equal to $n^{*}(n-1)$, where " $n$ " is the existing number of weighted edges (Borgatti, Everett, \& Freeman, 2002). The densities are an indicator of how well communication channels in the network are able to let information flow out to the network's participants (Morrison, 2002). Figure 4 shows the variation in the global network measure of cohesion (i.e., overall density) across variables and meeting layers.

If we evaluate the densities' trends across meetings within social signals the values can be interpreted as follows. At a meta-verbal level, the objective indication of the information flow in our study is turn-taking (TTK). We can observe that the communication spike is marked in the latter part of meeting one and it soothes around a steady value of 40 turns taken by the team members for every layer. The TTK average densities' pattern has two possible explanations. It is either an indicator equally distributed number of ties (i.e., making up communication channels that send information evenly to all connected team members) or it shows equally weighted edges (i.e., as intense communication between connected team members).

At a strictly nonverbal behavioral level, the other four variables show similar trends across meetings. Proximity (PRX) is the necessary variable for computing TTK; the rationale is that the team members have to be in each other's proximity to be able to share and assimilate information. Hence the PRX densities have similar trends to TTK. F2 F densities however, have a low variation with the exception of meeting 2 where they seem to spike in layers six and ten to an average of 10-15 pings of face-to-face interaction (i.e., approximately 10-15 seconds per episode; for a more detailed evaluation see Appendix 1) The positive mirroring relative to the total interaction time has very different patterns across meetings but the two variables are very consistent with each other within meetings (i.e., BMM and PM densities vary in a similar manner within meetings). 

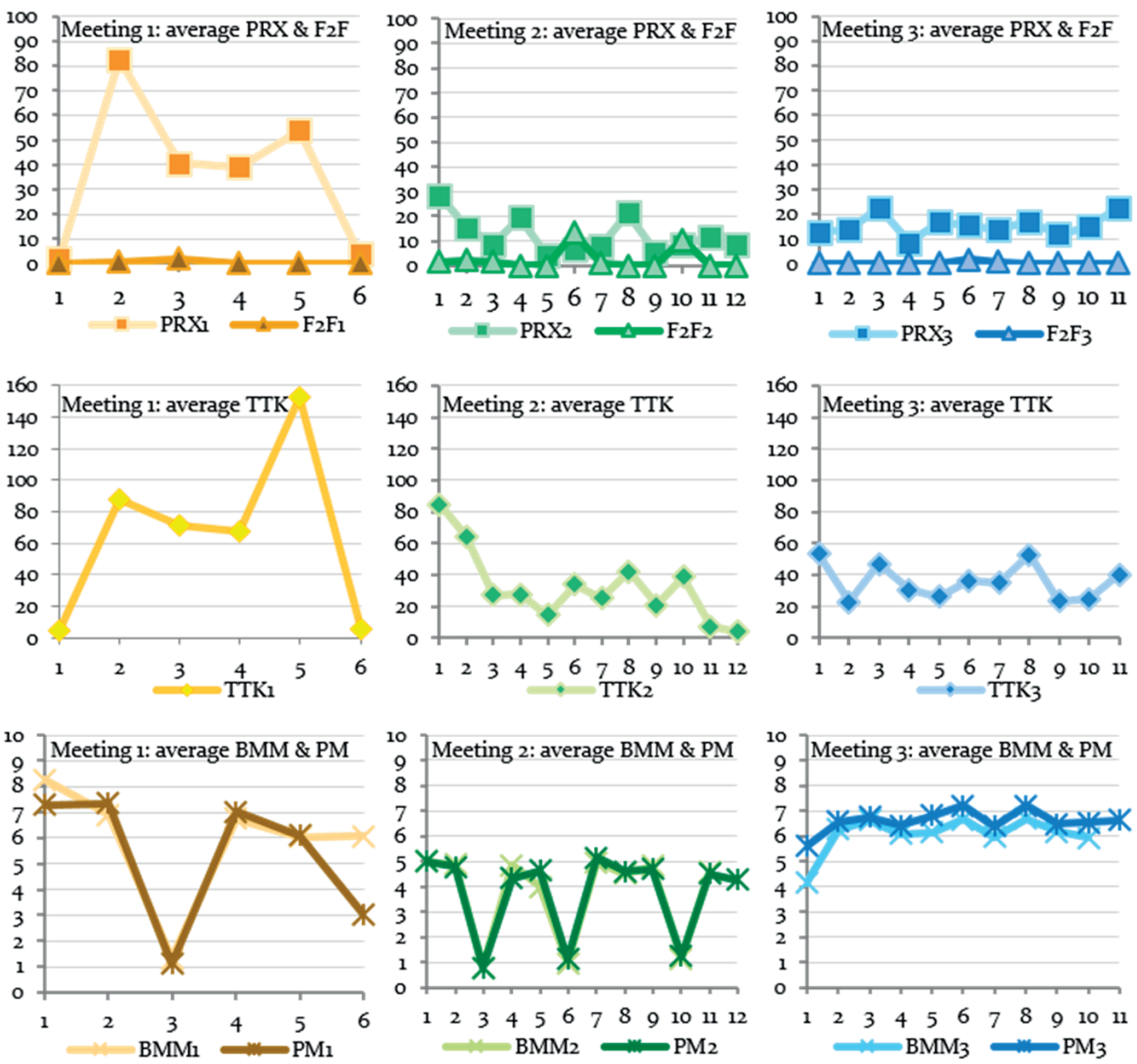

Figure 4. Topological overview of average densities per episode per variable: six layers in meeting 1 (in yellows), 12 layers in meeting 2 (in greens), and 11 layers in meeting 3 (in blues).

The maximum percentage of positive mirroring was BMM in meeting three, episode one with an average density of 9 per cent. If we evaluate the densities' trends across social signals within meetings the values can be interpreted as follows. In meeting one, the signals have growing densities in the beginning of the interaction and then a sudden drop after episode two. They pick up again and spike around layers four and five and plummet towards the end of the meeting. In meeting two, the variations in density varies in a less dramatic manner, but there seem to be some spikes in layers four, six, and ten-eleven. Lastly, in meeting three layers three and eight show slight increases in density across variables. 


\subsection{Multiple Regressions with Quadratic Assignment Procedure (MRQAP)}

We conducted multiple regressions with the quadratic assignment procedure (MRQAP) to analyze the effects of the independent variables on the dependent variable (Kapucu \& Hu, 2016). Specifically, we answer our research question by assessing to which extent turn-taking, proximity, face-to-face, body movement mirroring, and posture mirroring ties respectively affect the averaged mutually assessed creativity. As our data is relational, QAP is the appropriate method, as it does not assume independence of observations (Quintane \& Kleinbaum, 2011). The quadratic assignment procedure (QAP) together with the multiple regression equivalents, MRQAP, was developed to detect structural similarities by comparing networks in terms of their correlation (Krackhardt, 1987, 1992). The procedures do not detect statistically significant changes in dynamic networks (i.e., over-time).

MRQAP has four steps. First it conducts a multiple regression across the cells of the dependent and the independent variable matrices to calculate the regression coefficients (Borgatti et al. 2002). Second, the rows and columns from the dependent variable are randomly permuted 10, ooo times (Harrison, Sciberras, \& James, 2011) within each layer to recalculate the regression coefficients and then the $\mathrm{R}^{2}$ values and standard error are computed (Hanneman \& Riddle, 2005; Krackhardt, 1988). Third, the algorithm then compares the coefficients computed in step 2 with those produced in the step 1 . Lastly, it calculates the number of random permutations required in step 2 to yield results as extreme as those calculated in the step 1 . A low similarity percentage in results found in step 2 relative to step 1 serves as evidence of a significant relationship (Raider \& Krackhardt 2001). To avoid autocorrelation and collinearity issues, we applied the Double Dekker Semi-Partialling method (Dekker, Krackhardt, \& Snijders, 2007).

We regressed the matrices of turn-taking, proximity, face-to-face, body movement mirroring, posture mirroring networks respectively against the creativity network using UciNET' s (Borgatti et al., 2002) multiple regression with quadratic assignment procedure (MRQAP) with Double Dekker Semi-Partialling and 10,0oo permutations to insure the robustness of our results (Harrison et al., 2011).

Unlike conventional statistical procedures (i.e., the ordinary least square regression) QAP does not assume the independence of observations; that makes this method appropriate for analyzing dyadic data. Statistical procedures such as multiple regression and correlation become available for network data by using QAP.

Another distinction between QAP and OLS is that they affect the results they render in two main ways. First, as it relies on permutation-based tests (Ferrin, Dirks, \& Shah, 2006; Gibbons, 2004) QAP regression cannot compute degrees of freedom and statistical power. Second, due to the limited number of ties in QAP the equivalent correlations or beta values could have different significance levels resulting in $\mathrm{R}^{2}$ values smaller than those rendered by when traditional OLS regressions (Gibbons, 2004). Some scholars go as far as to put forward that the $\mathrm{R}^{2}$ values computed with QAP are irrelevant (e.g., Gibbons, 2004). We present the results below in Table 2. Given the above, the $p$ value is the critical output of the QAP regression results rather than the $\mathrm{R}^{2}$ value (Gibbons 2004; Zagenczyk, Purvis, Shoss, et al., 2015). 
Table 2. Models: regression coefficients and model fit

\begin{tabular}{lc|c|c} 
& $\begin{array}{c}\text { Meeting 1- } \\
\text { Exploration and ideation }\end{array}$ & $\begin{array}{c}\text { Meeting 2 - } \\
\text { Prototyping the idea }\end{array}$ & $\begin{array}{c}\text { Meeting 3 - } \\
\text { Pitch of final prototype }\end{array}$ \\
\hline PRX & -0.075 & -0.155 & -0.104 \\
F2F & 0.000 & $0.129^{*}$ & 0.043 \\
TTK & 0.010 & 0.350 & $-0.506^{*}$ \\
BMM & 0.154 & 0.049 & 0.084 \\
PM & $-0.266^{* *}$ & -0.309 & 0.012 \\
Intercept & 43.559 & $48.05^{*}$ & 33.265 \\
\hline R-Square & 0.114 & 0.061 & 0.264 \\
Adj R-Sqr & $0.085^{* *}$ & $0.029^{* *}$ & $0.208^{* *}$ \\
P-Value & 0.034 & 0.047 & 0.031 \\
\hline \hline
\end{tabular}

Note: ${ }^{* *} p<0.01 ;{ }^{* *} p<0.05 ;{ }^{*} p<0.1$

UciNET's multiple regression with quadratic assignment procedure (MRQAP) with double Dekker semi-partialling and 10 ooo permutations (Harrison et al., 2011).

In meeting one, the variables explain almost nine percent of the variation in mutually assessed creativity. The only significant coefficient is the amount of positive mirroring which decreases the alter-perceived creativity $(\beta=-0.266, p<0.05)$. In meeting two, the variables explain three percent of the creativity variation. The face-to-face interaction time has a marginally significant enhancing effect $(\beta=0.129, p<0.01)$. In meeting three, only the nonverbal behavior explains 20 per cent of the variation in mutually assessed creativity; in this case turn-taking has marginally significant strong negative effect $(\beta=-0.506, p<0.1)$. To shed more light on the results above, we plotted the graphs for each of the significant variables in Figures 5, 6, and 7. As mentioned in Descriptives for Global Network Measures, the pattern of densities is either an indicator equally distributed number of ties or it shows equally weighted edges. In the graphs below we zoom into the dyadic measurements of posture mirroring $[\mathrm{PM}]$ in meeting 1 , of time spent face-to-face $\left[\mathrm{F}_{2} \mathrm{~F}\right]$ in meeting 2 , and respectively of turn-taking [TTK] in meeting 3 over the composing layers (previously described in Table 1).

Figure 5 presents the network topology of the relative time spent in positive mirroring at dyad level. In this meeting, PM varies in layers 1, 3, and 6 from a noticeable pattern that occurs in 2, 4, and 5. The directions as well as the weights of the edges seem to vary in the former set. In the latter case, the graphs indicate fully connected positive posture mirroring networks with very similar weights.
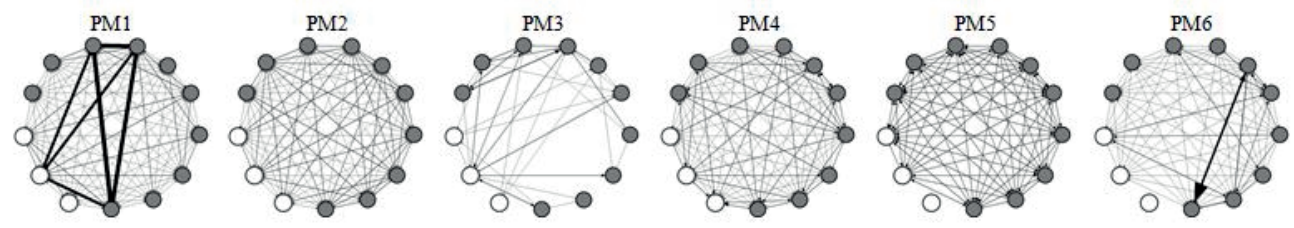

Figure 5. Meeting 1 - Topological overview of networks depicting the relative amount time spent in positive posture mirroring in the six composing layers: [PM 1] to [PM 6] 
Figure 6 shows the dyadic evolution of the time spent face-to-face. Layers 1, 2, 7, and 10 stand out in terms of both edge count as well as edge weight, layers 3, 4, 6, 9, and 11 show low connectivity and edge weight, whereas in layers 5,8 , and 10 the subjects did not face each other at all.
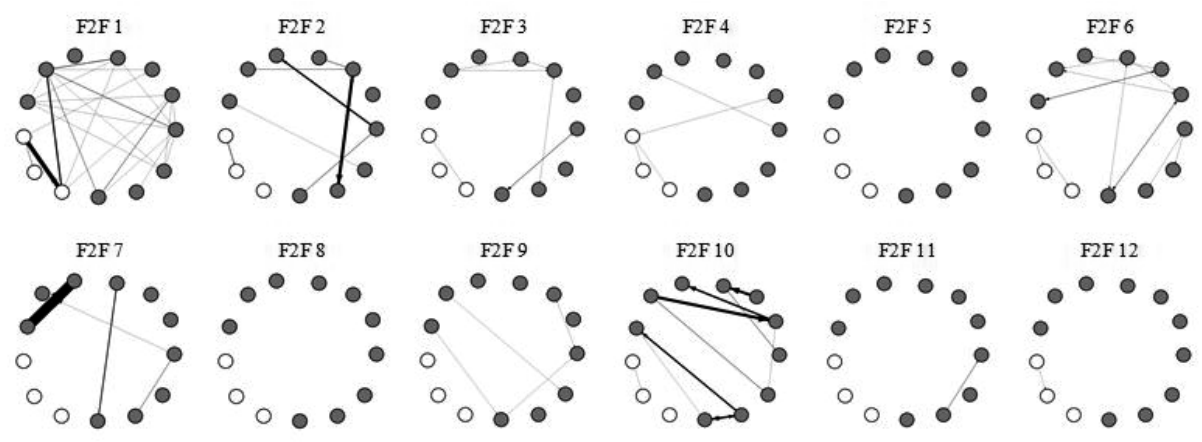

Figure 6. Meeting 2 - Topological overview of the networks depicting the amount time spent face-to-face in the twelve composing layers: $\left[\mathrm{F}_{2} \mathrm{~F}_{1}\right]$ to $\left[\mathrm{F}_{2} \mathrm{~F}_{12}\right]$

Figure 7 describes the communication patterns of meeting three where we observed the strongest negative effects on the communication outcome. The dyadic relationships do not seem to vary much in weight or number, but the values are high; they also do not seem conducive to effective communication outcomes as they are fully connected.
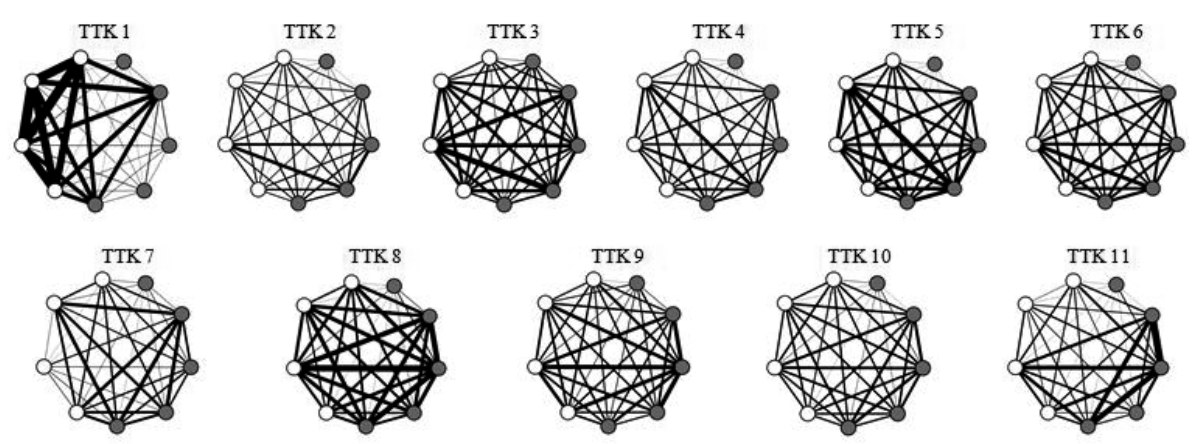

Figure 7. Meeting 3 - Topological overview of the networks depicting the amount turns taken in the eleven composing layers: [TTK 1] to [TTK 11]

The method we employed (MRQAP) compared the networks in terms of their correlations to detect structural similarities (Krackhardt, 1987, 1992). Having taken a global network perspective with the MQAP, the purpose of this section is to analyze the sociometric features at a dyadic (local) level.

\subsection{Multi-class Classification}

In the previous section we assumed a linear relationship between social signals and creativity. However, as introduced in Chapter 2, for studies such as the one investigated 
where (1) the theoretical foundations are insufficient for constructing hypotheses and (2) the structure and complexity of the data are known, it is difficult to establish a priori whether the relationship between variables is linear or polynomial. In such cases, a suitable approach is to employ supervised classification to learn models with fewer predefined boundaries imposed on the separability of targets. In our case, this means the prediction of different brackets or bins of dyadic creative performance based on social signal data.

To this end, we started by taking the network dyads over time as cases, the sociometric features and the layer number as input features, and the (dyadic) creativity scores as a target variable. Table 3 below depicts the resulting relationships of participant "o1-1586" (see "dyad.from") with all team members (see "dyad.to") during meeting one as they occurred in layer number 2 (i.e., while performing the second task).

To focus the analysis, the target variable (i.e., creativity) was split into four classes; by splitting the creativity variable at the average twice sequentially we obtained four mutually exclusive target classes per meeting, namely very high, high, low, and very low. The objective of the analysis is assigning cases (i.e., the dyads) to one of the four creativity categories given the input features (i.e., the social signals).

Table 3. The first dyad in layer two of meeting 1: relationships unpivoted from the social signals and creativity matrices (raw values before normalization)

\begin{tabular}{|c|c|c|c|c|c|c|c|c|}
\hline \multicolumn{2}{|c|}{ Cases } & \multicolumn{6}{|c|}{ Input features } & \multirow{2}{*}{$\begin{array}{c}\text { Target } \\
\text { variable } \\
\text { Creativity }\end{array}$} \\
\hline Dyad.from & Dyad.to & TTK & PRX & FTF & BMM & PM & Layer & \\
\hline o1-1586 & o1-1586 & 74 & o & o & o & o & 2 & o \\
\hline o1-1586 & 02-1587 & 94 & 106 & o & 7 & 8 & 2 & 14 \\
\hline 01-1586 & 03-1588 & 134 & 122 & o & 7 & 9 & 2 & 20 \\
\hline o1-1586 & o4-1589 & 136 & 152 & o & 7 & 8 & 2 & 24 \\
\hline o1-1586 & 05-159o & 77 & 10 & o & 6 & 8 & 2 & 37 \\
\hline $01-1586$ & o6-1591 & 110 & 18 & o & 7 & 8 & 2 & 17 \\
\hline 01-1586 & o7-1593 & 99 & 60 & o & 7 & 7 & 2 & o \\
\hline o1-1586 & o8-1594 & 150 & 108 & o & 7 & 8 & 2 & 15 \\
\hline o1-1586 & $09-1595$ & 57 & 35 & o & 7 & 8 & 2 & 25 \\
\hline $01-1586$ & $10-1597$ & 62 & 30 & o & 7 & 6 & 2 & 38 \\
\hline 01-1586 & 11-1598 & 102 & 12 & o & 6 & 7 & 2 & 26 \\
\hline o1-1586 & 12-1599 & 83 & 112 & o & 6 & 5 & 2 & 24 \\
\hline o1-1586 & $13-1600$ & 107 & 37 & $\mathrm{o}$ & 6 & 6 & 2 & o \\
\hline
\end{tabular}

Specifically, the analysis process was twofold; (1) we first look at the input features by assessing the extent to which each of the social signals and layers (therein "features") predict creativity; (2) we then create a predictive model by classifying the features (i.e., the five social signals and the layers) into one of the four levels of creativity.

\subsubsection{Forward Feature Selection (FFS)}

We investigated the role each sociometric variable in determining dyadic creativity using a forward feature selection (FFS) technique. This technique is used when dealing with multiple features and relatively few data points. FFS can be used as feature selection procedure for 
model refinement as well as a "quasi-factor analysis" technique to establish the role of specific features in predicting the target variables.

This technique has two premises; (1) it identifies some features that are redundant in explaining the target variable (Bermingham, Pong-Wong, Spiliopoulou, et al., 2015) and (2) it ranks the features in order of their improving explanatory accuracy. Notably, the identified set of features does not comprise the assumptions of a predictive model making it more suitable for revealing relationships between the features. We used cross-fold validation to account for the small sample size to reduce overfitting. The method works as follows. The set of observations is randomly divided into $k$ groups (or folds) of approximately equal size. The first group is treated as a validation set whereas the remaining k-1 groups are used to train the machine learning algorithm. Thus, the former set is the estimation set and the latter is the validation set. The algorithm is repeated $\mathrm{k}$ times, until each group has been both part of the estimation and validation sets. We chose the feature ranking cut-off point via crossfold validation with 10 iterations (see Appendix 4).

\subsubsection{Multi-class Classification with a Random Forest Classifier}

We predict the likelihood model of any feature (i.e., the five social signals and the layers) pertaining to any of the four levels of creativity. Since we split the target variable (i.e., creativity) into four classes (i.e., very high, high, low, and very low) the classification is thus multi-class.

The analysis was conducted using the Random Forest (or Random Decision Forest) classifier from the WEKA machine learning library (WEKA, 2017a). The Random Forest classifier assigns (or maps) the features to the target variables and it is composed of multiple decision trees. In a decision tree model, the "leaves" represent the target variables and the "branches" represent conjunctions of the input features that lead to those target variables. The objective is to create a model that predicts the value the different levels of creativity (i.e., the target variables) based on the five social signals and the layers (i.e., the input features). Usually, the algorithms for creating decision trees work top-down (imagining the "trees' roots" at the top), by naturally choosing a feature at each step that best splits the set of items (Rokach \& Maimon, 2005). The downside of such methods of determining variable importance is that the trees become biased towards features with more levels. We circumvent this drawback by growing unbiased trees (Painsky \& Rosset, 2017; Strobl, Boulesteix, \& Augustin, 2007) using cross-fold validation as partitioning procedure for the Random Forest models (for a detailed explanation of the cross-validation technique see "Feature evaluation" and Appendix 4).

The models were run using the WEKA multi-class classification framework (WEKA, 2017b). The random forest classifier took consensus from the 10 decision trees (i.e., obtaining a random forest composed of 10 distinct randomly created decision trees) and was selected for its computational efficiency and ability to produce good fit quality (Breiman, 2001a). The models were run with all features (again with $\mathrm{k}=10$ cross-fold validation), and scored using precision, recall and F1 measures, as well as with reference to overall model accuracy, the aim being to maximize these values (the former three should also be relatively balanced across classes). 
To improve class balance beforehand, we also ran the synthetic minority class oversampling technique - SMOTE, described in Chawla, Bowyer, Hall, and Kegelmeyer (2002). All variables were normalized using simple min-max normalization (i.e. in this case, rescaled to o-1) to give each variable (including layer number) equal predictive weighting.

\subsection{Results}

\subsubsection{Forward Feature Selection (FFS)}

The feature selection results describe the role each feature (i.e., social signals and layers) plays in predicting the target variable (i.e., creativity). As mentioned, the procedure identifies the feature that has the biggest impact on predicting the target and is then iteratively adding the remaining features with an optimization goal, in our case, maximizing the overall model accuracy. Table 4 depicts the results of the feature evaluation for each of the three meetings. This iterative method starts with no feature in the model. With each of the 10 iterations (see "Feature evaluation"), the algorithm adds the feature that most improves the accuracy of the model. From the bottom to the top row we show the features in the order they were added to the model.

In meeting 1, PRX was the most important feature and thus firstly added and it represents $38.43 \%$ of the total accuracy. The second most important was TTK and the accuracy increased from $38.43 \%$ to $60.75 \%$. A less dramatic increase was due to adding the third feature, PM, up to $70.18 \%$. BMM added $3.65 \%$ change and, lastly, the layer improved the accuracy by $3.19 \%$. F2F added almost no change to the accuracy (i.e., o.10\%) therefore we excluded it from the forthcoming analysis.

Table 4. Feature selection results across meetings

\begin{tabular}{|c|c|c|c|c|c|c|c|c|c|c|c|}
\hline $\begin{array}{c}M \\
1\end{array}$ & $\begin{array}{l}\text { Added } \\
\text { feature }\end{array}$ & Accuracy & Change & $\begin{array}{c}M \\
2\end{array}$ & $\begin{array}{c}\text { Added } \\
\text { feature }\end{array}$ & Accuracy & Change & $\begin{array}{c}M \\
3\end{array}$ & $\begin{array}{r}\text { Added } \\
\text { feature }\end{array}$ & Accuracy & Change \\
\hline $6^{\text {th }}$ & FTF & $77.12 \%$ & $0.10 \%$ & $6^{\text {th }}$ & FTF & $63.99 \%$ & $0.00 \%$ & $6^{\text {th }}$ & FTF & $82.08 \%$ & $0.15 \%$ \\
\hline $5^{\text {th }}$ & Layer & $77.02 \%$ & $3.19 \%$ & $5^{\text {th }}$ & Layer & $63.99 \%$ & $2.09 \%$ & $5^{\text {th }}$ & BMM & $81.93 \%$ & $0.30 \%$ \\
\hline $4^{\text {th }}$ & BMM & $73.83 \%$ & $3.65 \%$ & $4^{\text {th }}$ & $\mathrm{PM}$ & $61.90 \%$ & $2.94 \%$ & $4^{\text {th }}$ & PM & $81.63 \%$ & $5.87 \%$ \\
\hline $3^{\mathrm{rd}}$ & PM & $70.18 \%$ & $9.43 \%$ & $3^{\mathrm{rd}}$ & BMM & $58.96 \%$ & $7 \cdot 38 \%$ & $3^{\text {rd }}$ & Layer & $75 \cdot 76 \%$ & $8.87 \%$ \\
\hline $2^{\text {nd }}$ & TTK & $60.75 \%$ & $22.32 \%$ & $2^{\text {nd }}$ & PRX & $51.58 \%$ & $16.04 \%$ & $2^{\text {nd }}$ & PRX & $66.89 \%$ & $21.10 \%$ \\
\hline $1^{\text {st }}$ & PRX & $38.43 \%$ & $38.43 \%$ & $1^{\text {st }}$ & TTK & $35.54 \%$ & $35 \cdot 54 \%$ & $1^{\text {st }}$ & TTK & $45.79 \%$ & $45.79 \%$ \\
\hline
\end{tabular}

In meeting 2, TTK was the most important feature and it marks $35.54 \%$ of the total accuracy. The second most important was PRX as the accuracy increased from $35.54 \%$ to $51.58 \%$. Thirdly, by adding the third feature, BMM, the accuracy grew by $7.38 \%$. PM added a $2.94 \%$ change and, lastly, the layer marginally enhanced the accuracy to $63.99 \%$. F2 F did not improve the model accuracy so we, again, removed it from the forthcoming analysis.

In meeting 3, TTK was again the most important feature marking almost half of the total accuracy (i.e., 45.79\%). The second most important feature was again PRX and the accuracy increased to $66.89 \%$. The third most important feature was the layer that enhanced the 
accuracy by $8.87 \%$. Lastly, BMM and $\mathrm{F}_{2} \mathrm{~F}$ had almost no impact on the accuracy with a combined change of $0.45 \%$ consequently we excluded them both from the classification.

F2F had a negligible influence on overall model accuracy across meetings most likely due to the low variance in data, while BMM had a negligible influence in the third meeting (although this feature contributed $3.65 \%$ and $7.38 \%$ change in model accuracy in the first and second meetings respectively). Notably, across all three meetings TTK and PRX are the first two loaded features and together account for $>80 \%$ of the overall model accuracy.

\subsubsection{Multi-class Classification with a Random Forest Classifier}

Following the forward feature selection, we conducted the classification with five input features for M1 and M2 (i.e., PRX, TTK, PM, BMM, and Layer) and four input features for $\mathrm{M}_{3}$ (i.e., TTK, PRX, Layer, and PM). Basic descriptive statistics are shown in Appendix 3 for each meeting in Table 7,8 , and 9 respectively.

In Figure 8 below we show a simple example of a classification with two possible outcomes. For the sake of exemplification, we can assume that the red section is homologous to very high and the green is high. The threshold is the value we selected for partitioning the classes, namely the average and grater.

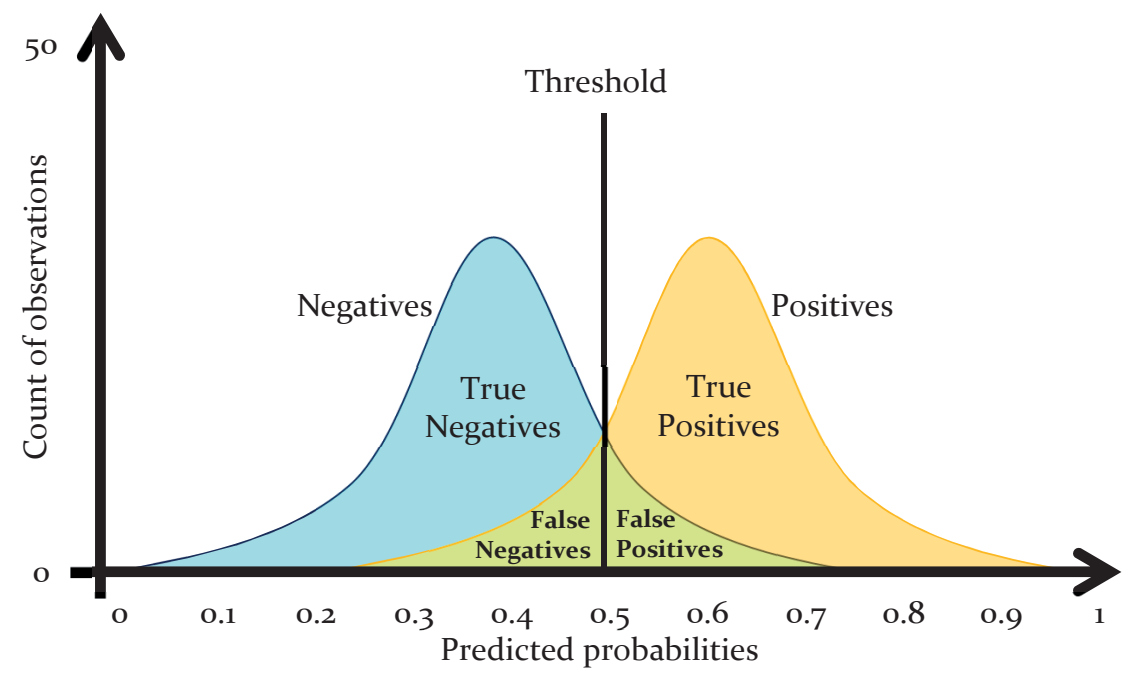

Figure 8. Example of predicted probabilities in a binary classification

The True Positive represent the cases where the model correctly predicted the actual class. The True Negatives values are the correctly identified negative cases. The False Positives are the result of the model incorrectly predicting the actual class. Similarly, the False Negatives are the incorrectly identified negative cases.

These are the main building blocks of the metrics used to evaluate our classification models. The "true" values are correctly classified cases, whereas the "false" values are misclassified 
classes. Another way to depict the probabilities is by plotting them in a $2 \mathrm{X} 2$ confusion matrix (i.e., error matrix) as in Figure 9 below.

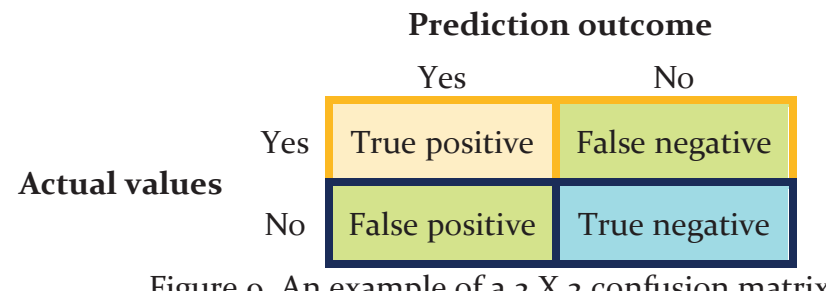

Figure 9. An example of a $2 \mathrm{X}_{2}$ confusion matrix

Since we performed a multi-class classification with four classes, the appropriate way to visualize the performance of the algorithm is as $4 \mathrm{X}_{4}$ confusion matrices as depicted in Table 5. Just as in Figure 9, the rows represent the number of instances in an actual class and the columns represent the number of instances in a predicted class. The diagonal elements represent the correct decisions (i.e., the correctly classified cases, either positive or negative). The areas above and below the diagonals show how many cases were confused (i.e. mislabeled) within each pair of classes. We can interpret the values in the confusion matrices by comparing each pair of classes as shown in Figure 9.

In Table 5 below, across all meetings the diagonals show correctly classified cases with the exception of the low creative ties in $\mathrm{M}_{3}$. Where the classifiers exhibit inter-class misclassification, the confusion matrices show less separability between the creative ties that were reported as low in $\mathrm{M}_{1}$ and M2, as well as between very high and low in M2.

Table 5. Confusion matrices for each meeting

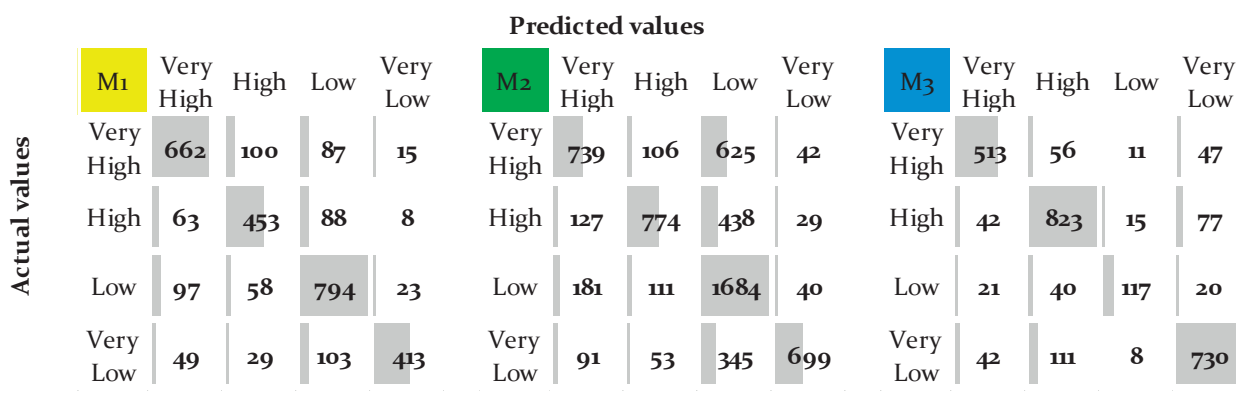

From the above metrics (i.e., True positive, True negative, False positive, and False negative) the classifier computes the model accuracy by dividing the number of correct predictions to the total number of predictions. Another metric is precision as it shows the proportions of positive identifications that were correct. In other words, precision is computed by dividing the True positive values to the sum of True and False Positive values.

The algorithm also computes the recall metric as the proportion of actual positives that was identified correctly by dividing the True positive values to the sum of True Positive and False Negative values. Alongside accuracy, the other measure of model performance is the F- 
measure (or F-score). The F-measure is the harmonic mean of precision and recall; the best value of the F-measure is 1 and the worst is o (Flach, 2012).

Overall model accuracy results are summarized in Table 6 . Classes are ordered by the target classes. We achieved strong overall accuracy, namely 76.33\%, 64.04\%, and 81.67\% for M1, M2, and $\mathrm{M}_{3}$ respectively. Recall (i.e., the true positive rate) and precision (i.e., positive predictive value) are strong for all classes (Powers, 2011), although slightly less for low and very low tie evaluations in M2. We discuss the results per meeting by reviewing the values in Tables 5 and 6.

Table 6. Model performance across meetings

\begin{tabular}{|c|c|c|c|c|c|c|c|c|c|c|c|c|}
\hline \multirow[b]{2}{*}{$\begin{array}{l}\text { Creativity } \\
\text { Score }\end{array}$} & \multicolumn{4}{|c|}{$\begin{array}{c}\text { Meeting } 1- \\
\text { Exploration and ideation }\end{array}$} & \multicolumn{4}{|c|}{$\begin{array}{c}\text { Meeting } 2- \\
\text { Prototyping the idea }\end{array}$} & \multicolumn{4}{|c|}{$\begin{array}{c}\text { Meeting } 3^{-} \\
\text {Pitch of final prototype }\end{array}$} \\
\hline & $\begin{array}{l}\text { Very } \\
\text { High }\end{array}$ & High & Low & $\begin{array}{l}\text { Very } \\
\text { Low }\end{array}$ & $\begin{array}{l}\text { Very } \\
\text { High }\end{array}$ & High & Low & $\begin{array}{l}\text { Very } \\
\text { Low }\end{array}$ & $\begin{array}{l}\text { Very } \\
\text { High }\end{array}$ & High & Low & $\begin{array}{l}\text { Very } \\
\text { Low }\end{array}$ \\
\hline Precision & 0.76 & 0.71 & 0.74 & 0.9 & 0.65 & 0.74 & 0.54 & 0.86 & 0.83 & 0.8 & 0.77 & 0.84 \\
\hline Recall & 0.77 & 0.74 & 0.82 & 0.7 & 0.49 & 0.57 & 0.84 & 0.59 & 0.82 & 0.86 & 0.59 & 0.82 \\
\hline F-measure & 0.76 & 0.72 & 0.78 & 0.78 & 0.56 & 0.64 & 0.66 & 0.7 & 0.82 & 0.83 & 0.67 & 0.83 \\
\hline $\begin{array}{l}\text { Model } \\
\text { Accuracy }\end{array}$ & \multicolumn{4}{|c|}{$76.33 \%$} & \multicolumn{4}{|c|}{$64.04 \%$} & \multicolumn{4}{|c|}{$81.67 \%$} \\
\hline
\end{tabular}

In meeting 1 , the accuracy reached $76.33 \%$. The value can be explained by the very high and high classes confusion, where 100 very high cases are misclassified as high. Also, a total of 278 cases were misclassified as low. The F-scores indicate that there are consistent patterns in the signals predicting the low and very low classes. Model performance is good which can be explained by the homogeneity of interactions during Mi.

In meeting 2, the accuracy reached $64.04 \%$ which is slightly lower. A total of 1408 cases were misclassified as False Positive low from across all the other three classes. Largely the F-scores are having small values but they have an ascending trend from very high to very low. An explanation for the poorer performance of this model is the heterogeneity in the sociometric data due to the overlapping sub-team tasks. These results however lend credence to the scores in $\mathrm{M}_{1}$ and $\mathrm{M}_{3}$ where there was less heterogeneity in social signals and thus obtaining higher validity.

In meeting 3, the accuracy reached the high value of $81.67 \%$ and the highest F-scores, except for the low class. One explanation is the very low number of True Positive values (i.e., 117) and a relatively high number of False Positives with the high class (i.e., 40). Model performance is very good which was also anticipated since $\mathrm{M}_{3}$ had no heterogeneity of interactions.

\section{Discussion}

The main objective of this study was to evaluate the effects of social signals on creativity in $\mathrm{B}_{2} \mathrm{~B}$ new service development. We addressed calls for research from the marketing literature on the interplay between firms and their customers (Cui and $\mathrm{Wu}, 2015$ ) by examining a $\mathrm{B} 2 \mathrm{~B}$ co-developing team in the ideation phase. We investigated both the verbal (i.e., creativity) and nonverbal (i.e., social signals) behaviors to gage a holistic view of the interactions within 
the NSD team. The second line of calls for research we answer is that on the creative outcomes of nonverbal aspects of communication (Onnela et al., 2014). Previous research on nonverbal communication was exclusively built upon tools that are known for their limitations and biases (e.g., self-assessments, observations, and audio-video coding, etc.) (Kozlowski, 2015).

The overarching findings are as follows. Even in this time-limited NSD phase, the dynamic leading to creativity is very complex. The joined NSD team exhibits different levels of creativity and social signals at different points in time and at distinct analysis levels (i.e., at team level and at dyad level).

Specifically, at team level we learn that while "diverging" in their idea exploration (M1), the more time team members spent positively mirroring their postures the lower they perceive each other's creativity. All through "idea emergence" (M2) the more time spent directly facing each other the more creative they evaluate each other. During "idea convergence" (M3) the distribution of the conversation channels negatively influenced the perceived creativity. Most importantly, overall, al social signals explain a significant variance in the perceived alter-creativity across all three meetings; this lends credence to the validity of the sociometers as a new methodological approach.

When we break down the global networks into their composing relationships (i.e., dyads) we find further patterns. Firstly, in the "idea divergence" (M1) being in each other's proximity and contributing to generating ideas play a crucial role in predicting the creative outcome; an extra layer of importance is added by the time team members spent positively mirroring each other's postures even given that $27 \%$ of the meeting was spent in sub-team activities. The social signals have, overall in $\mathrm{M} 1$, a strong predictive power of the different creativity levels. Secondly, in the "idea emergence" (M2) the team members take on sub-team tasks and work separately for the most part. The dyads are thus heterogeneously divided and randomized over time depending on the tasks at hand. In this particular instance we learn that the communication patterns alongside proxemics play the most important role in determining the four levels of creativity. Contrasting to $\mathrm{M}_{1}$, here we observe that the supplementary role in explaining the creative behavior is played by the time spent in mirroring each other's energy levels (or body movement). The social signals are able to predict the four levels of creativity with moderate accuracy even under the conditions of subtask based randomized dynamics that made up $42 \%$ of the meeting duration. Lastly, in the "idea convergence" $\left(\mathrm{M}_{3}\right)$ the team had to decide for an incremental or radical new service prototype. The negotiation was guided by discussion points (or tasks), but the outcome was up for deliberation. In this meeting all discussions were held in plenum. We learn that, alongside the conversation patterns and proxemics, the actual topics discussed (i.e., the moments in time when the tasks were covered) had the crucial role in explaining the creative outcome. The predictive power of the social signals was highly accurate and at its strongest of all three meetings.

\subsection{Methodological Contributions}

There is little evidence in previous literature on the effects of multiple types of interactions among different actors (Kapucu \& Hu, 2016) and the studies that examined such multiplex relationships investigate the macro level actors (i.e., organizations) (Kapucu, 2009). Drawing 
support from the social-psychology literature on the theoretical precedent of nonverbal communication effects on creativity we employ wearable technology to collect objective such multiplex nonverbal signals that we investigate with subjective assessments of creativity at meso level (i.e., team). However, this global (i.e., network) evaluation does not detect statistically significant changes in networks over-time; we supplement the methodology with a state-of-the-art supervised machine learning exercise to uncover the effect of social signals and tasks on creativity at dyad level.

Results show varied effects for the capacity of individual nonverbal variables as predictors of creativity across meetings at team level, with different body language variables demonstrating predictive power in earlier meetings, and conversational features demonstrating predictive power in the last meeting. However, in shifting to a discriminative prediction approach (i.e., at dyad level), results showed that both conversational and body language features represent strong proxies for creative outcome prediction, with turn-taking and proximity alone contributing an average of $29.87 \%$ improvement to models, peaking at an average accuracy of $74.40 \%$ across meetings.

However, three main challenges still remain when it comes to such studies. Firstly, during the data collection, the participants reported comfort issues while wearing the devices due to the devices' weight (i.e., approximately 100 grams). While the sensors and the motherboard are light, the battery makes the badge rather heavy as it is built to support the continuous collection of 8 hours of data.

Secondly, the machine learning rendered discriminative models have two main issues, namely transparency and post-hoc interpretation (Mittelstadt, Russell, \& Wachter, 2018). Transparency explains the inner working of the model, while the post-hoc explanations detail how the model performs (Lepri, Oliver, Letouzé, Pentland, \& Vinck, 2017; Lipton, 2016; Montavon, Samek, \& Müller, 2017). The former can be addressed (as we have in this chapter as well as in Chapter 4) by explaining the overall model performance, the performance of the parameters in the feature selection process, and the training algorithm (Lepri et al., 2017). However, the latter can be problematic since discriminative models don't provide direct causation. The textual and/ or visual explanation of how and why a model performs provide a qualitative assessment of the relationship between features selected and the model's classification results (Ribeiro, Singh, \& Guestrin, 2016). At the moment there is heightened debate in the academic community on best practices of post-hoc explanations (Mittelstadt et $\mathrm{al}, 2018)$.

\subsection{Theoretical Contributions}

We contribute broadly to the extant literature on team processes by employing objective measurements collected with high-frequency sampling rates (Kozlowski, 2015) over time (de Montjoye, et al., 2014). Specifically, we contribute to the team creativity literature by showing that in each of the three meetings (that had distinct goals) different combinations of nonverbal behaviors explain the perceived mutually assessed creative ties.

In meeting one (i.e., the people-focused exploration and ideation phase), at team level, we show that the relative time spent in positive body movement decreases the alter-perceived creativity. In other words, if team members exhibit posture mirroring in most of the 
meeting's duration the perceived creativity decreases. We contribute to the existing theory on kinesics by showing that the time spent in relative positive body movement (e.g., the amount of time passed while the level of energy of the ego and alter increase almost synchronously) inhibits perceived creativity. As there is an inextricable link between speech and body movement (i.e., our cognitive processes are embodied) (Feldman, Feldman, \& Rimé, 1991) we can assume body movement convergence to be a valid proxy for consensus (Bernieri \& Rosenthal, 1991) or 'group think'. At dyadic level, the communication channels unfolding over time (i.e., turn-taking) and the time spent in proximity are pivotal for predicting the alter-perceived creativity.

In meeting two (i.e., the task-focused idea prototyping phase), at team level, the time spent in face-to-face enhances the creative assessment of team members. This finding does not hold for the dyadic level where the face-to-face time had no impact on creativity. The theoretical boundary condition is thus that the time each dyad spends facing each other in the prototyping phase influences perceived creativity only when most if not all possible dyads are present.

In meeting three (i.e., the task- and people-focused pitch of final prototype phase), at team level, the social signals have a strong predictive power. We show that turn-taking has significantly strong negative effect in this project phase. The same holds for the dyadic assessment with the addition that the tasks are strong predictor of how team members evaluate each other's creativity. Thus, in the converging stage the communication distribution negatively influences creativity; we argue that this implies a communication style in the realm of negotiation (which is crucial to reaching consensus before the team moves on to the next project phase) rather than idea generation.

\subsection{Practical Implications}

Our study is preponderantly relevant for professional service providers that need an in-depth overview of the outcomes of involving customers as co-developers in the new service development processes (Chang \& Taylor, 2016). We outline the importance of nonverbal behavior in affecting as well as predicting creativity in the ideation phase of the process, proving that creativity is the outcome of complex social interactions. We also show the importance of the tasks at hand, and the time of the project for the creative outcome.

\subsection{Limitations}

This study has some limitations that future research can address. First, due to the scope of the study we did not account for team members' individual attributes. Future studies should consider the micro-level factors as well as build upon our findings and describe the influence of team diversity, team longevity, team size, etc. on team outcomes (i.e., performance, learning, etc.). Second, our study is limited to one team developing the idea of a service in the ideation phase. Future research should investigate the entire project span. Third, we did not provide the tasks a pivotal role in our research design. Researchers should investigate specifically the effect of the service design tools on the interaction patterns. Fourth, we considered the multiplex networks and the dyads as units of analysis thus not fully addressing the interplay between the customer and firm. 


\subsection{Future Research Directions}

Future research should discriminate between three main types of ties: customer-tocustomer, customer-to-firm, and firm-to-firm to better explain the co-developing touchpoints in the new service development process. Fifth, future research should consider a larger sample size as our sample of 13 team members renders a very low generalizability of results. Lastly, building upon the previous suggestion (i.e., understanding the process), future research should then design and deploy interventions to improve the processes and ultimately boost creativity among co-developing new service development teams. 


\section{Appendix 1. Firmware configuration}

\section{Accelerometer:}

Interval to read accelerometer values: $\quad 0.05 \mathrm{~s}$

Interval to store accelerometer values: $\quad 0.5 \mathrm{~s}$

Infrared (IR):

Interval to send IR beacon: 15

Random value to +/- IR beacon interval: $\quad$ o.1s ( = actual detections happen at an interval of +- o.1s)

Bluetooth (BT):

Interval for BT inquiries (used for BT detections): Random value to +/- BT inquiry interval: $\quad \begin{aligned} & 5 \mathrm{~s}(=\text { actual detections happen at an } \\ & \text { interval of }+-5 \mathrm{~s})\end{aligned}$

The maximum amount of time before the BT inquiry process is halted $5.120 S$

(< inquiry interval):

Limit BT detections to sociometric badges only:

\section{Microphones:}

Audio data to skip on start: 105

Audio sensitivity: $\quad 3 \mathrm{x}$

2ch (back and

Channels: $\quad$ front): $\quad$ Audio storage feature (back): o.5s

Audio storage feature (front): $0.5 \mathrm{~s}$ 
Appendix 2. Example of an unfolded multiplex matrix with three nodes and three layers and the respective graphs

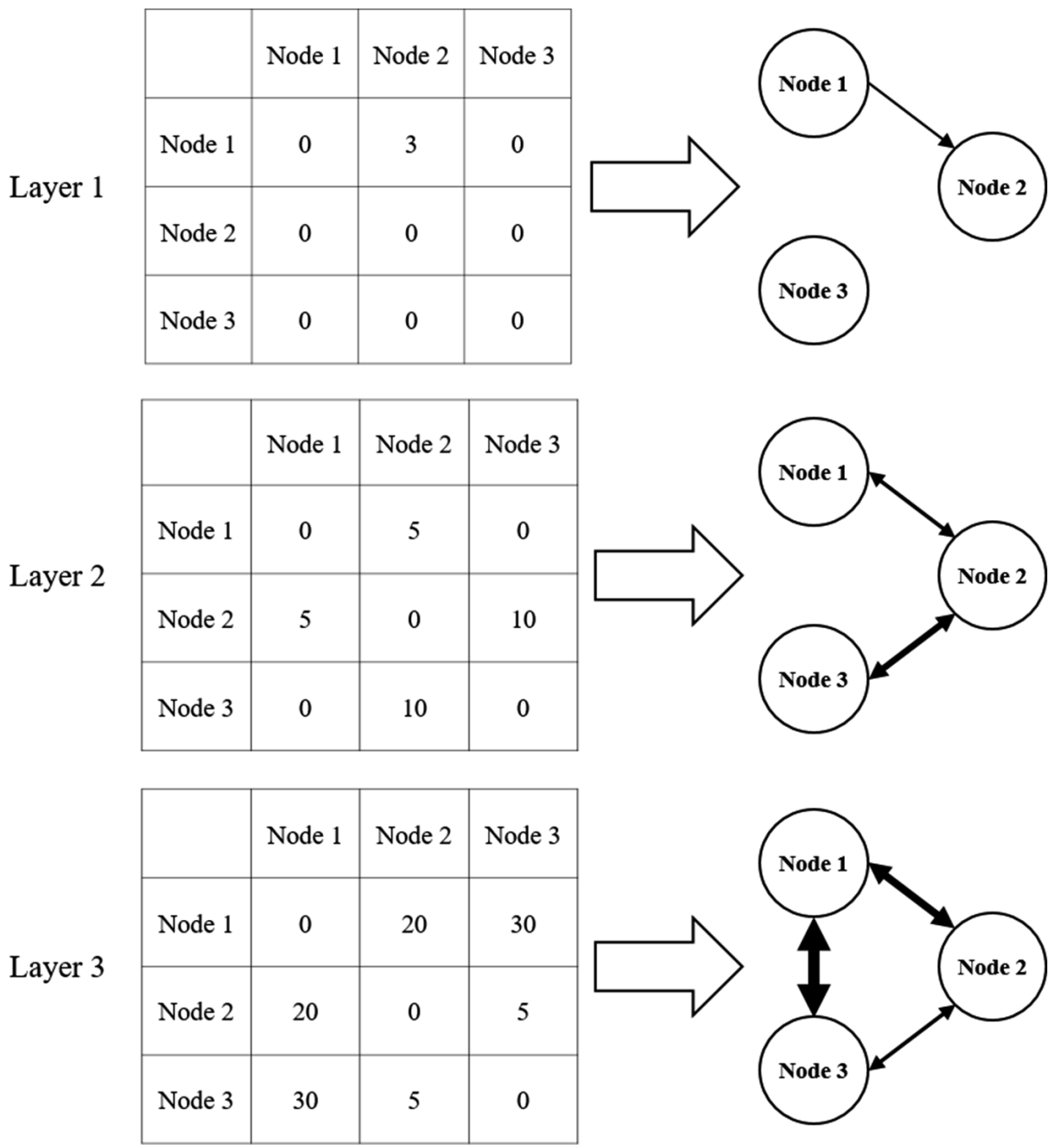




\section{Appendix 3. Descriptive statistics}

Table 7. Descriptives for $\mathrm{M}_{1}$

\begin{tabular}{|c|c|c|c|c|c|c|c|c|}
\hline Class & $\begin{array}{l}\text { Mean } \\
\text { BMM }\end{array}$ & $\begin{array}{c}6 \\
\text { BMM } \\
\end{array}$ & $\begin{array}{c}\text { Mean } \\
\text { PM }\end{array}$ & $\begin{array}{c}\mathbf{6} \\
\mathbf{P M}\end{array}$ & $\begin{array}{c}\text { Mean } \\
\text { PRX }\end{array}$ & $\begin{array}{c}6 \\
\text { PRX }\end{array}$ & $\begin{array}{c}\text { Mean } \\
\text { TTK }\end{array}$ & $\begin{array}{c}\mathbf{6} \\
\text { TTK }\end{array}$ \\
\hline Very high & 6.22 & 11.35 & 5.08 & 5.00 & 35.57 & 42.54 & 75.44 & 75.64 \\
\hline High & 5.87 & 10.77 & 4.38 & 2.88 & $37 \cdot 30$ & 40.75 & 70.75 & 74.95 \\
\hline Low & 5.38 & 10.34 & 4.38 & 4.89 & 37.63 & 42.47 & 51.01 & 58.78 \\
\hline Very low & 3.94 & 10.99 & 6.07 & 15.28 & 23.14 & 39.56 & 64.84 & 88.54 \\
\hline
\end{tabular}

\begin{tabular}{|c|c|c|c|c|c|c|c|c|}
\hline Task & $\begin{array}{l}\text { Mean } \\
\text { BMM }\end{array}$ & $\begin{array}{c}6 \\
\text { BMM }\end{array}$ & $\begin{array}{c}\text { Mean } \\
\text { PM }\end{array}$ & $\begin{array}{c}6 \\
\text { PM }\end{array}$ & $\begin{array}{c}\text { Mean } \\
\text { PRX }\end{array}$ & $\begin{array}{c}6 \\
\text { PRX }\end{array}$ & $\begin{array}{c}\text { Mean } \\
\text { TTK }\end{array}$ & $\begin{array}{c}\mathbf{6} \\
\text { TTK }\end{array}$ \\
\hline 1 & 7.64 & 19.63 & 6.72 & 16.62 & 1.67 & 1.52 & 4.52 & 6.00 \\
\hline 2 & 6.37 & 2.01 & 6.78 & 2.19 & 76.39 & 63.09 & 83.53 & 34.01 \\
\hline 3 & 1.21 & 2.27 & 1.04 & 1.95 & 37.41 & 28.71 & 85.57 & 95.28 \\
\hline 4 & 6.24 & 2.17 & 6.47 & 2.31 & 36.17 & 21.55 & 63.92 & 28.43 \\
\hline 5 & 5.57 & 3.10 & 5.67 & 3.12 & 49.83 & 35.11 & 143.57 & 87.36 \\
\hline 6 & 5.59 & 16.67 & 2.78 & 6.75 & 3.43 & 3.41 & 6.62 & 14.12 \\
\hline
\end{tabular}

Table 8. Descriptives for M2

\begin{tabular}{ccc|cc|cc|cc} 
Class & $\begin{array}{c}\text { Mean } \\
\text { BMM }\end{array}$ & $\begin{array}{c}\mathbf{6} \\
\text { BMM }\end{array}$ & $\begin{array}{c}\text { Mean } \\
\text { PM }\end{array}$ & $\begin{array}{c}\mathbf{6} \\
\text { PM }\end{array}$ & $\begin{array}{c}\text { Mean } \\
\text { PRX }\end{array}$ & $\begin{array}{c}\text { PRX } \\
\text { PRean }\end{array}$ & $\begin{array}{c}\text { TTK } \\
\text { TTK }\end{array}$ \\
\hline Very high & 3.19 & 3.88 & 2.99 & 3.42 & 9.71 & 12.91 & 26.89 & 47.59 \\
High & 4.30 & 4.00 & 4.13 & 3.60 & 12.97 & 16.36 & 38.06 & 57.68 \\
Low & 3.97 & 4.05 & 4.04 & 3.91 & 13.47 & 17.73 & 33.98 & 56.84 \\
Very low & 2.10 & 3.11 & 2.40 & 3.55 & 7.78 & 14.08 & 32.58 & 57.98 \\
\hline \hline
\end{tabular}

\begin{tabular}{ccc|cc|cc|cc} 
Task & $\begin{array}{c}\text { Mean } \\
\text { BMM }\end{array}$ & $\begin{array}{c}\mathbf{6} \\
\text { BMM }\end{array}$ & $\begin{array}{c}\text { Mean } \\
\text { PM }\end{array}$ & $\begin{array}{c}\mathbf{6} \\
\text { PM }\end{array}$ & $\begin{array}{c}\text { Mean } \\
\text { PRX }\end{array}$ & $\begin{array}{c}\mathbf{6} \\
\text { PRX }\end{array}$ & $\begin{array}{c}\text { Mean } \\
\text { TTK }\end{array}$ & $\begin{array}{c}\mathbf{6} \\
\text { TTK }\end{array}$ \\
\hline 1 & 4.62 & 3.74 & 4.61 & 3.61 & 26.30 & 18.95 & 79.56 & 60.89 \\
2 & 4.50 & 3.57 & 4.43 & 3.57 & 14.33 & 13.75 & 60.76 & 49.45 \\
3 & 0.72 & 1.89 & 0.74 & 1.91 & 8.15 & 20.75 & 34.51 & 73.80 \\
4 & 4.46 & 3.51 & 4.01 & 3.22 & 17.89 & 14.79 & 26.50 & 23.54 \\
5 & 3.67 & 3.64 & 4.27 & 4.07 & 3.64 & 3.75 & 13.84 & 12.65 \\
6 & 1.02 & 2.51 & 1.12 & 2.77 & 6.18 & 15.49 & 39.48 & 83.11 \\
7 & 4.57 & 3.74 & 4.74 & 4.16 & 7.51 & 6.32 & 23.87 & 19.85 \\
8 & 4.15 & 3.28 & 4.25 & 3.42 & 19.62 & 19.42 & 39.93 & 41.34 \\
9 & 4.44 & 3.98 & 4.32 & 3.92 & 5.27 & 5.08 & 19.75 & 18.10 \\
10 & 1.06 & 2.65 & 1.15 & 2.91 & 7.81 & 20.44 & 45.54 & 98.83 \\
11 & 4.22 & 3.41 & 4.17 & 3.38 & 10.99 & 10.66 & 7.04 & 6.47 \\
12 & 4.41 & 5.98 & 3.94 & 3.60 & 8.08 & 8.04 & 3.73 & 4.65 \\
\hline \hline
\end{tabular}


Table 9. Descriptives for $\mathrm{M}_{3}$

\begin{tabular}{|c|c|c|c|c|c|c|}
\hline Class & $\begin{array}{c}\text { Mean } \\
\text { PM }\end{array}$ & $\begin{array}{c}6 \\
\mathbf{P M}\end{array}$ & $\begin{array}{c}\text { Mean } \\
\text { PRX }\end{array}$ & $\begin{array}{c}6 \\
\text { PRX }\end{array}$ & $\begin{array}{c}\text { Mean } \\
\text { TTK }\end{array}$ & $\begin{array}{c}\mathbf{6} \\
\text { TTK }\end{array}$ \\
\hline $\begin{array}{l}\text { Very } \\
\text { high }\end{array}$ & 5.61 & 3.13 & 11.75 & 10.53 & 20.98 & 20.18 \\
\hline High & 6.55 & 5.04 & 15.75 & 10.23 & $39 \cdot 32$ & 22.19 \\
\hline Low & 6.88 & 1.86 & 9.71 & 7.18 & 32.24 & 21.02 \\
\hline Very low & 5.10 & 3.11 & 13.78 & 10.66 & 36.33 & 32.15 \\
\hline Task & $\begin{array}{c}\text { Mean } \\
\text { PM }\end{array}$ & $\begin{array}{c}\mathbf{6} \\
\mathbf{P M}\end{array}$ & $\begin{array}{c}\text { Mean } \\
\text { PRX }\end{array}$ & $\begin{array}{c}6 \\
\text { PRX }\end{array}$ & $\begin{array}{c}\text { Mean } \\
\text { TTK }\end{array}$ & $\begin{array}{c}\mathbf{6} \\
\text { TTK }\end{array}$ \\
\hline 1 & 5.04 & 9.29 & 11.32 & 11.63 & 51.70 & 56.62 \\
\hline 2 & 5.83 & 2.68 & 12.06 & 6.83 & 21.44 & 11.19 \\
\hline 3 & 5.99 & 2.67 & 20.10 & 9.71 & 43.53 & 18.26 \\
\hline 4 & 5.70 & 3.67 & $7 \cdot 41$ & 5.25 & 28.49 & 21.75 \\
\hline 5 & 6.06 & 2.95 & 14.94 & 7.97 & 24.86 & 16.04 \\
\hline 6 & 6.41 & 2.99 & 13.90 & 8.01 & 33.84 & 16.24 \\
\hline 7 & 5.67 & 2.73 & 12.11 & 7.69 & 32.67 & 20.45 \\
\hline 8 & 6.40 & 2.86 & 15.25 & 13.85 & 48.70 & 26.02 \\
\hline 9 & 5.75 & 2.70 & 10.40 & 8.21 & 21.93 & 11.60 \\
\hline 10 & 5.80 & 2.87 & 13.16 & 11.10 & 23.26 & 12.91 \\
\hline 11 & 5.89 & 2.57 & 20.15 & 13.56 & 38.05 & 23.16 \\
\hline
\end{tabular}




\section{Appendix 4. Example of $K$-fold $(K=10)$ validation for a subset of 7 dyads}

\begin{tabular}{|c|c|c|c|c|c|c|c|c|}
\hline $\begin{array}{l}\quad \text { Example } \\
\text { Dataset } \\
\text { K-fold } \\
(\mathrm{K}=10)\end{array}$ & $\begin{array}{c}\text { Dyad } \\
1\end{array}$ & $\begin{array}{c}\text { Dyad } \\
\mathbf{2}\end{array}$ & $\begin{array}{c}\text { Dyad } \\
3\end{array}$ & $\begin{array}{c}\text { Dyad } \\
4\end{array}$ & $\begin{array}{c}\text { Dyad } \\
5\end{array}$ & $\begin{array}{c}\text { Dyad } \\
6\end{array}$ & $\begin{array}{c}\text { Dyad } \\
7\end{array}$ & \\
\hline Iteration 1 & Test & Train & Train & Train & Train & Train & Train & => Performance \\
\hline Iteration 2 & Train & Test & Train & Train & Train & Train & Train & => Performance \\
\hline Iteration 3 & Train & Train & Test & Train & Train & Train & Train & => Performance \\
\hline$\ldots$ & $\ldots$ & $\ldots$ & $\ldots$ & $\ldots$ & $\ldots$ & $\ldots$ & $\ldots$ & $=>$ Accuracy \\
\hline Iteration 8 & Train & Train & Train & Train & Test & Train & Train & $=>$ Performance \\
\hline Iteration 9 & Train & Train & Train & Train & Train & Test & Train & => Performance \\
\hline Iteration 10 & Train & Train & Train & Train & Train & Train & Test & $=>$ Performance \\
\hline
\end{tabular}

We used cross-fold validation to account for the small sample size to reduce overfitting. The method works as follows. The set of observations is randomly divided into $k$ groups (or folds) of approximately equal size. The first group is treated as a validation set whereas the remaining $k-1$ groups are used to train machine learning algorithm. Thus, the former set is the estimation set and the latter is the validation set. The algorithm is repeated $k$ times, until each group has been both part of the estimation and validation sets. 
This chapter is based on Francu, E., van den Bossche, P., Lucas, B., Segers, M., Lemmink, J. (2019). For Your Ties Only: Creative Activation in Innovation Project Teams. A Network Alignment Approach. Working Paper

An early version of this paper was presented at $9^{\text {th }}$ SERVSIG Conference (Services Special Interest Group of the American Marketing Association), June 2016 in Maastricht, The Netherlands and at the $25^{\text {th }}$ Annual Frontiers in Service Conference, June, 2016, in Bergen, Norway. 


\section{Chapter 4}

For Your Ties Only 


\begin{abstract}
Innovation teams reach a creative outcome through a creative problem-solving process. In previous studies, creativity was inferred from self-assessed cognitive proxies. Conversely, we set out to understand team interaction patterns that lead to creative problem solving by looking at how creative relationships (ties) align with objective social behaviors over the span of two innovation projects.

We measured (1) creative behaviors through dyadic mutual evaluation creativity collected during project debriefings and (2) the interaction patterns with wearable measurement devices containing Bluetooth and infrared sensors, a tri-axial accelerometer, and two microphones; these devices are used to derive social signal variables computed from dyadic second-by-second level measurement. We coin the concept of "creative activation" as the convergence between creative ties and social signals. We employ dynamic network alignment to examine the intersection of real-time behaviors (i.e., creative problem solving and social signals) generated by two new service development (NSD) teams, and their participating customers during actual project meetings. We introduce network alignment as a new method to social sciences as it facilitates the formalized comparison of networks with common constituent node and edge types and different topological structures.

The method reveals that, by aligning the networks we are able to (a) show network topological evidence of creative activation in teams, and, (b) isolate patterns in social signals (i.e. interaction synchrony) at different points in time that contribute most to this result.
\end{abstract}

Keywords: Teams, social signals, wearable technology, network alignment, supervised classification 


\section{Introduction}

Extant literature acknowledges customer involvement as a catalyst of innovation performance and of improving the firm's knowledge on customers' needs to develop tailored solutions (Chang \& Taylor, 2016; Cui \& Wu 2015; Zhou, Hong, \& Liu, 2013). In practice, an increasing number of companies $(\mathrm{He}, 2 \mathrm{O13})$ rely on project teams to generate creative ideas and to convert them into useful technology, products or services (e.g., Chesbrough, 20o6; Hoegl \& Parboteeah, 2006; Salas, Shuffler, Thayer, Bedwell, \& Lazzara, 2015; Thamhain, 2003). Moreover, the customers who part-take in these project teams are an essential source of insights for the development of new products and services (Iyer \& Davenport, 2008). In this respect, companies' performance is highly dependent on the process they employ to engage customers to co-create value (Schneider, Ehrhart, Mayer, et al., 2005). However, there are still two prominent gaps in the innovation and human resource literature. Firstly, involvement of customers in the innovation processes is under investigated (Cui \& Wu 2015, p. 17). Secondly, research should deepen the human resource management perspective on creativity and on evaluating and understanding the dynamics of teams in projects' processes (De Clercq, Mohammad Rahman, \& Belausteguigoitia, 2015; Sue Chan \& Hempel, 2016; van Woerkom \& Meyers, 2015).

There is a consistent and growing interest in creativity and innovation as broader concepts (Brown, 2016; Grabner, 2014; Grabner \& Speckbacher, 2016; Schwarz, 2015), especially through the frames of human resource management (Heyden, Reimer, \& Van Doorn, 2015; Scott, Heathcote, \& Gruman, 2011) and management (Alexander \& Van Knippenberg, 2014; Harvey, 2014). However, no research has sought to deepen managerial understanding of such processes with objective behavioral proxies, such as by leveraging wearable-enabled computational social science and workplace analytics (e.g. Bersin et al., 2016; Montanari et al., 2017) to help understand and optimize team creativity in an organizational setting, despite calls for research to move in this direction (George et al., 2016). Recently, technologies such as remote sensing and wearables have been highlighted as paving the way for a research cross-over from big data and data science research to management research (George et al., 2016).

Our study aims to address these gaps and to uncover patterns in the team synergy between the firm and participating customers. We introduce the concept of "topology-based creative activation", building on the interactional synchrony literature stream, where we investigate the convergence of nonverbal social signals between social actors as a predictor of various group outcomes. In our case, this outcome is a creative moment arising from the codevelopment interaction between team members, with teams consisting of staff and customers. Specifically, we investigate the nonverbal social interactions (NVSI) between B2B actors involved in the new service development (NSD) process that engender 'creative activation'.

Therefore, the emphasis of this study is advancing managerial understanding of codevelopment with customers in innovation processes by investigating creativity implementation in organizational projects with objective behavioral proxy data. We define creativity, in contrast to innovation, as the process of "coming up with fresh ideas for 
changing products, services, and processes so as to better achieve the organization's goals" (Amabile \& Pratt, 2016).

Importantly, we conduct this study with an emphasis on longitudinal field studies and highfrequency sampling rates (Kozlowski, 2015), enabled by data collection with wearable technology. Our study also focuses on professional service firms, a context which is particularly attractive for human resource management (HRM) researchers (Richter, Dickmann, \& Graubner, 2008), given the dependence of these firms on people and knowledge resources (Fu, Flood, Bosak, Rousseau, Morris, \& O'Regan, 2017). Additionally, we examine the entire project process, from idea generation, through prototype development, to launch preparation (Chang \& Taylor, 2016).

\section{Literature Review}

A recent, prominent trend in management practice is companies' shift away from centralized product and service development structures, and towards customer-centric organizational formats which actively involve customers and end users and are supported by individually empowered project teams (McDowell, Agarwal, Miller, Okamoto, \& Page, 2016). It is imperative for organizations to develop solutions (products, services, or hybrid offerings) and innovate in very short cycle times (Alavi, \& Leidner, 2001) to remain competitive. These project teams are successfully developing new solutions if the knowledge managing processes are effectively coordinated (Rubenstein-Montano, Liebowitz, Buchwalter, et al., 2001). Acquiring, absorbing, and utilizing knowledge through customer participation requires (1) high quality insights from the knowledge provider (i.e., customers) (Mahr, Lievens, \& Blazevic 2014), (2) the actors' (i.e., customers and firms') to be highly motivated and capable to exchange knowledge, and (3) the firms' to efficiently gather the creative insights and morph them into tangible outcomes (Szulanski, 1996; Von Hippel 1994).

As discussed in Chapter 1, in the particular case of new services being developed with the involvement of customers (i.e., 'customer involvement as co-developer' or CIC), these settings require customers work closely NPD employees to jointly develop new solutions (Cui \& Wu, 2015). Similar collaborations have focused management and human resources researchers' attention on team creativity (Alexander \& Van Knippenberg, 2014; Gilson \& Shalley, 2004; Gloor, Almozlino \& Inbar et al., 2014; Perry-Smith \& Shalley, 2014; Schwarz, 2015) and the information systems researchers' on problem-solving (e.g., (RubensteinMontano, et al., 2001). The two perspectives can be unified under Amabile's (1996) distinct concept of creative problem solving whereby team creativity is considered in context and is dependent on the task at hand.

A growing body of literature focuses on how the dynamic social interactions shape the creative process (Brennan \& Enns, 2015; De Jaegher, Di Paolo, \& Gallagher, 2010; Heerey, 2015). This new perspective acknowledges the importance of the individuals' contribution but stresses that the individual creative potential manifests itself in the social interaction between team members (Perry-Smith \& Shalley, 2003). Exemplarily, Perry-Smith and Shalley (2014) investigate the social aspects of team creativity, while Gilson and Shalley (2004) explore task definition within a team creativity context. This has also led to further research on the interactive aspects of team creativity. For example, Sosa (2011) examine the factors 
influencing the emergence of creative interactions, and Sosa, Gargiulo, and Rowles (2015) investigate the disruption brought about by informal communicative networks in a new product development context.

Nonverbal social interactions have been shown previously to serve as highly relevant predictors of individual characteristics (Giles, 1979; Helfrich, 1979; Robinson, 1979; Scherer, 1989; Scherer \& Scherer, 1981; Smith, 1980). As covered extensively in Chapter 3, such research has centered largely on the fundamental understanding of how nonverbal social signals carry and convey information about speaker's personality, affective states, age, socio-economical background among other characteristics. These social cues are communicative or informative signals. The communicative signals refer to verbal communication, whereas the informative cues refer to nonverbal communication. These social signals, also known as affective signals, convey information about social interactions such as emotions and attitudes (Poggi \& Francesca, 2010) and help individuals understand others' attitudes by their gestures (Pease \& Pease, 2016). We emit these evolutionary residuals (Pentland, 2008) through our gestures, overall body language, facial expressions (Baur et al., 2013), and relative distance from others (Poggi and Francesca, 2010) while engaged in social interactions. Thus, our communication success depends not only on explicit content, but also on the implicitly conveyed social signals (Baur et al., 2013). Similarly, at group level, social signals have also been proven to be good predictors of social group outcomes (Scherer, 1979b; Shmueli et al., 2014) this research stream has focused on collaboration quality and team performance (de Montjoye et al., 2014; Pentland, 2012; Woolley, Chabris, Pentland, et al., 2010). It is thus remarkable that, to date research has not addressed the effects of nonverbal behavior on creative outcomes.

In terms of social structures, previous research has examined dyadic relations (Eberly, Holley, Johnson, \& Mitchell, 2011), with Barry and Crant (2000) examining micro-level communication at this level, as well as social network perspectives, which has included research in innovation, such as research by Perry-Smith and Mannucci (2017). Such structural perspectives are advancing due to developments in quantified-self and computational social science, which in turn have given rise to topics such as "the quantified workplace" (Mashhadi, Kawsar, Mathur, Dugan, \& Shami, 2016), "office analytics" (Montanari, Nawaz, Mascolo, \& Sailer, 2016), or "people analytics" (Bersin et al., 2016), a field pioneered by the work of Choudhury and Pentland (2004). Pentland (2012) discusses the ability to move beyond a subjective sense of "esprit de corps", by leveraging the ability of technology to capture complex communicative cues which are otherwise largely passively and unconsciously processed by humans when interacting.

In terms of applied contexts of teams within organizations, Pentland (2012) argues that such subjective and intuitive assessments of a team 'clicking' might be better captured not by the spoken interaction, but rather by the deeper communicative structure. This can be compared with the work of Harvey and Kou (2013), in their study of collective engagement with creative tasks, where they identified convergent decision making as a basis for achieving a creative outcome. Schwartz and Teach (2000) characterize the idea generation phases as being underpinned by divergent thinking, leading to an implementation phase underpinned by convergent thinking (Conway \& McGuinness, 1986). Additionally, Bernieri \& Rosenthal 
(1991) assert that behavioral convergence serves as a valid proxy for cognitive convergence. More recently, Perry-Smith and Mannucci (2017) discuss idea elaboration and idea championing as key phases in innovation processes.

\section{Research Questions and Research Aim}

As covered in the previous section, we consider team creativity a social and dynamic process of creating new and valuable ideas in context. We carry over the conceptual underpinnings into the methodology of the study by considering the team being made up by composing dyads of relationships between each pair of team members. Given this level of conceptualization, the appropriate approach is to take a dyadic or network perspective as opposed to placing the individual at the level of analysis. We coin the concept of 'creative activation' as the topology convergence of team creativity and nonverbal social signals between social actors.

Our network 'activation' perspective is thus supported by (a) considering collective cognitive convergence as a basis for creative outcomes in teams, and (b) considering social structural perspectives as a basis for understanding the micro-level (and often subtle) communication mechanisms. To date, activation within social networks has been explored within the context of social network influence (Jack, 2005; Yang, Chawla, Walter Licht, \& Dong, 2016). Compounding the literature on social creativity with that on nonverbal social interactions, we investigate the interactions between $\mathrm{B}_{2} \mathrm{~B}$ actors involved in the new service development (NSD) process that bring about 'creative activation'. We thus formulate the following research questions:

RQ1: Can aligning dyadic mutual evaluation (DME) and nonverbal social interactions (NVSI) networks produce evidence of the link between NVSI topology and 'creative activation'?

RQ2: Which social signals contribute the most to creative activation?

We address these research questions by measuring NVSI from two NSD teams and their participating customers (i.e., second-by-second dyadic social signals) collected using realtime wearable measurement devices. We aggregate this dyadic data at set time intervals, to represent the NVSI data as a network structure, comprising nodes, representing individual meeting participants (see for illustration: Yu, Blocker, Sir, Hallbeck, et al., 2016), with separate directed and weighted edge sets for each variable, networks where variables are represented as links connecting participants (body movement mirroring [BMM], posture mirroring $[\mathrm{PM}]$, volume mirroring $[\mathrm{VM}]$, frequency mirroring $[\mathrm{FM}]$, time spent in proximity $[\mathrm{PRX}]$ and time spent face-to-face $\left.\left[\mathrm{F}_{2} \mathrm{~F}\right]\right)^{7}$.

\footnotetext{
7 The variables are computed on the theoretical basis described in Chapter 3 in section 2.2.1. Effects of Nonverbal Communication
} 


\section{Method}

\subsection{Participants and Setting}

We conducted the field study during the entire span of the projects (ideation, development, and launch preparation) of two NSD projects in a Western European city. A service innovation research consultancy compiled the multidisciplinary team for either project and carried the projects out in collaboration with the respective $\mathrm{B}_{2} \mathrm{~B}$ clients (a regional education service provider and a medical service provider). Our sample consists of the aforementioned project teams. The first project team (hereafter "TG") consisted of 19 team members - three consultancy representatives, four client representatives, six students, and six related experts. The goal of this project was to innovate the service offering. The second project team (hereafter "TM") consisted of 16 team members - four consultancy representatives, a client representative, nine students, and two external experts. The goal of this project was to update the company's supply chain network.

The projects started one month apart and then took place in parallel. Their total duration was three, respectively four months (including the winter holidays comprised in the timespan). Each team had weekly joint meetings lasting approximately four hours; we thus tracked and measured a total of eight and nine meetings respectively.

Some of the team members had to gather insights and tackle tasks outside of these weekly team meetings. We acknowledge the pivotal importance of acquiring knowledge outside the boundary of the project team. However, the aim of this study is to uncover the interaction patterns of all team members attending each project meeting, as that was the scene of information sharing leading to creative activations. We acknowledge that, while covering all the possible interactions happening face-to-face, this study does not follow the inception, transformation, and implementation of actual ideas outside of project meetings. We consider the mutually evaluated dyadic creativity scores as ground truth for NVSI convergence which only took place during the joint project meetings.

\subsection{Meeting Format}

The consultancy representatives outlined the projects' desired outcomes in collaboration with the respective $\mathrm{B}_{2} \mathrm{~B}$ clients. They also managed the projects' processes and moderated the weekly project meetings that spanned over three to four hours. The team composition varied between meetings, thus we take an analysis approach focused on topological structure, rather than 'tracking' individuals. For confidentiality reasons we do not include the actual content or agenda of the meeting. We thus defined the structure of each meeting by splitting it in 30-minutes sessions. (This was empirically defined based on establishing a sufficient network connectivity threshold after comparing different time increments; we discovered that smaller aggregations did not produce sufficient levels of connectivity.). Table 6 in Appendix 1 shows a brief overview of each meeting and their respective sessions, with (from left to right), meeting identifiers, meeting start time, and meeting end time.

\subsection{Data Collection Procedure}

NVSI data was collected with sociometric badges described in Choudhury and Pentland (2004). The devices contain Bluetooth and infrared sensors, a tri-axial accelerometer and two microphones. From the raw sensor data (dyadic, second-by-second), we compute the NVSI 
variables. Prior to the projects' kick-offs, we have set up the sociometers. To avoid measurement errors, we assigned a badge to each participant for the entire project span by using the badges' unique IDs to insure the measurement consistency (i.e., making sure that an ID represents the same exact team member across projects and across project meetings), but also as credentials for the post-meeting survey (i.e., to enable linking the objective sociometric data to the subjective survey data).

The lead researcher participated in the projects' kick-off meetings and informed the team members about the purpose and procedure of our study, and about their required involvement. At the end of our information sessions, we handed out consent forms for participating in the study; all team members read and signed these documents. We then proceeded to handing out the sociometers, instructed the participants on their specifications, mentioning that the badges were programmed not to collect and log any personally discernible data to ensure their privacy and the projects' confidentiality. Then the institute's representatives took over the meetings' proceedings. Before the start of the next project meetings, we discreetly handed out the badges to all attendees. At the end of every meeting, we collected the sociometers, sent out the creativity survey with a five-day deadline to avoid recollection biases (Schacter, 1999), and downloaded the data from the badges. We employed the same procedure for every one of the project meetings.

\subsection{Variables' Operationalization and Plan for Analysis}

Similar to the data management approach we took in Chapter 3, each set of links (i.e., edge set) is separated into network layers, which can be treated both as individual networks, and as a composite of individual networks, producing a multigraph (see: Figure 1 and Zippo, Storchi, Nencini et al., 2013). Meeting participants also complete a dyadic mutual evaluation (DME) exercise, where each participant rates their creative ties with every other participant with regard to creative outcomes during the meetings, as part of project debriefing, informed by research precedent for social-network-based peer evaluation (Luria \& Kalish, 2013). We then employ network alignment for topological comparison between the DME and NVSI networks to produce evidence of the link between NVSI topology and 'creative activation'.

From the raw objective sensor data (dyadic, second-by-second), we compute the relational NVSI variables. As described in the Meeting format, we empirically defined sessions (i.e., episodes or layers) within each meeting to be able to assess the alignment of NVSI networks with the creativity network over time. We compared different time increments and subsequently established a sufficient network connectivity threshold at 30 minutes per session, as smaller aggregations did not produce sufficient levels of connectivity for network alignment. We then aggregated the dyadic, second-by-second data as network structures depicting 30-minutes time increments. Thus, each variable for each session is a network depicting the individual meeting participants as nodes and their ties (relationships) as separate directed and weighted edge sets connecting the nodes.

From the Bluetooth raw data, we computed the time spent in relative proximity [PRX]; the infrared data served the basis for calculating the time spent face-to-face [ $\left.\mathrm{F}_{2} \mathrm{~F}\right]$. From the voice features we extracted the amount of speech turns the interlocutors exchanged (i.e., turntaking) [TTK] and the amount of variation in the mirroring of their voices' volume (namely, 
volume mirroring $[\mathrm{VM}])$. From the tri-axial accelerometer data we extracted the variation in the mirroring of body movement [BMM] (or 'energy') and of posture [PM].

The former three, PRX, F2F, and TTK are exported as interval variables with values greater than o aggregated at the set 30-minutes interval. The latter three, VM, BMM, and PM are exported as minute-by-minute-split datasets per session marking the instances when mirroring happened (be it negative or positive) on a scale from -1 to 1 . Thus, we needed to further aggregate them to be able to align them with the other variables. We were interested in understanding the amount of variability relative to the mean (which in our case is o and stands for no mirroring) in either direction. We observed empirically that some dyads had an increasing standard deviation complementary to a decreasing mean (due to the negative values) when just calculating the mean of all observations per dyad at the 30 second aggregation. We thus decided to calculate a stabilization coefficient (SC) (Liu \& Zheng, 1989) as our measure of mirroring distribution. The definition of stabilization coefficient is just the reciprocal of variation coefficient, mean divided by standard deviation. Such a coefficient has a simple expectation and a simple variance, and is an asymptotically unbiased estimator and a consistent estimator of its true value. Furthermore, the coefficient of stabilization has an asymptotic normality. Due to its statistical advantages, the coefficient of stabilization is easy to be tested statistically.

Consequentially, the SC of the mirroring variables (e.g., VM, BMM, PM) was computed by calculating (1) the average negative mirroring, (2) the average positive mirroring, where $o$ describes no mirroring, (3) the stabilization coefficient from negative to positive with the values obtained in steps (1) and (2)

$$
S C=\frac{\left(M_{n e g}+M_{p o s}\right) / 2}{\left|M_{\text {pos }}-M_{\text {neg }}\right| / \sqrt{2}}
$$

where $M_{\text {neg }}=\bar{M}$, if $M \in[-1,0)$ and $M_{\text {pos }}=\bar{M}$, if $M \in(0,1]$

If we consider the distinct means instead of the SC the mirroring scores are overestimated and the mirroring dispersion is overestimated. For a detailed explanation of the computation of the three mirroring variables see Appendix 2.

The complete dataset was made up of six types of NVSI (i.e., PRX, F2F, TTK, BMM, PM, and VM) for each of the teams with 19 and 16 team members respectively. Each NVSI is separated into layers (one for each 30-minute increment within a meeting), which can be treated both as individual networks, and together, producing a multigraph or a multiplex network (Zippo et al., 2013). The dataset for TG was comprised by the 19 team members, the six NVSI, and the 78 sessions adding up to 4211 dyads (or 468 matrices). The dataset for TM was comprised of the data collected from the 16 team members, the same six NVSI, and the 64 sessions adding up to 3455 dyads (or 384 matrices).

The NVSI data is best explained visually. Figure 1 below shows a real example network from our data collection. Nodes represent individual sociometric badges, and thus the meeting participants. Red edges represent BMM, green edges represent PM, blue edges represent TTK, with self-loops indicative of self-turns. Orange edges represent VM. In this particular time bracket, $\mathrm{F}_{2} \mathrm{~F}$ interactions did not exist, thus this edge set is not represented. 


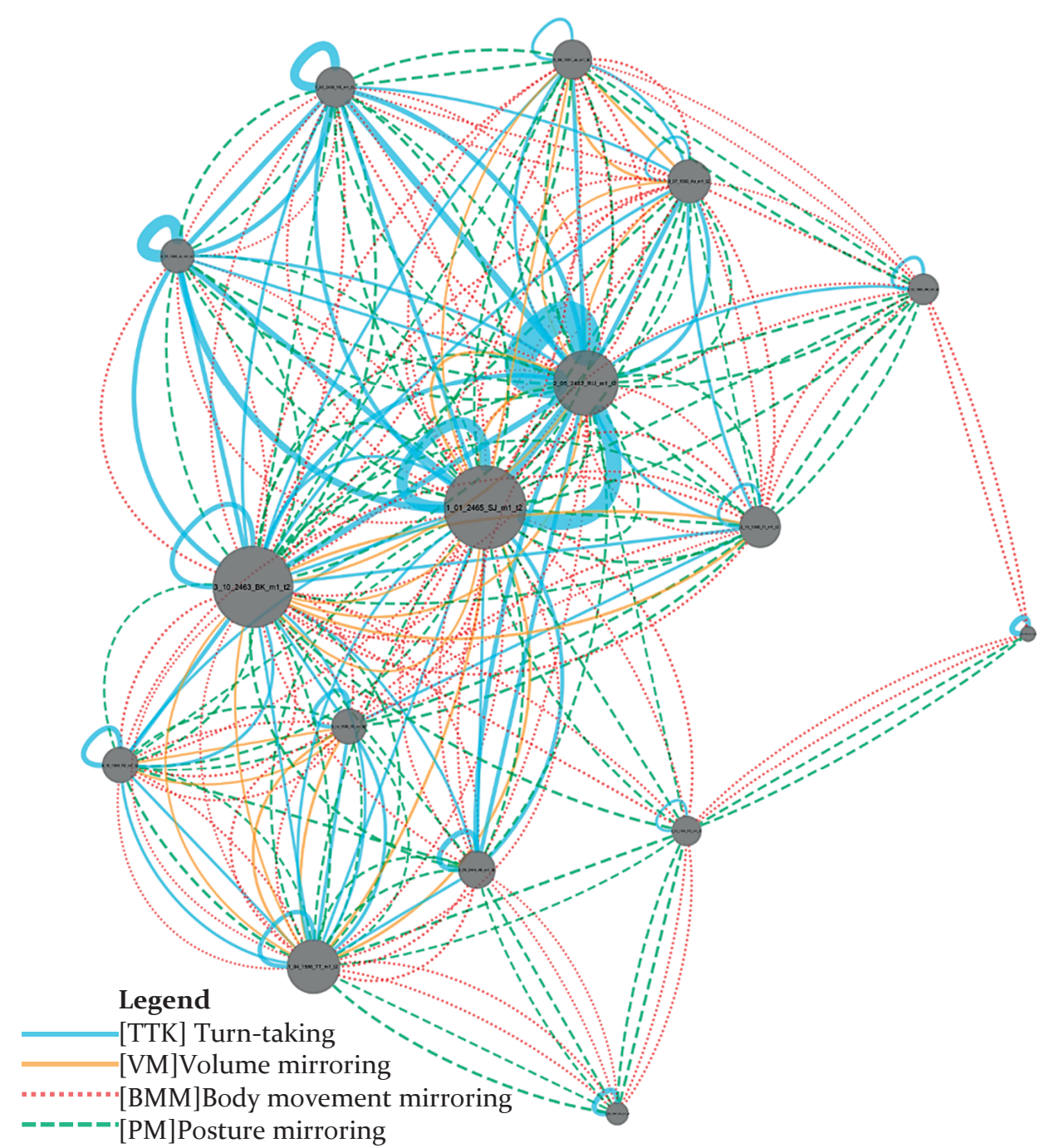

Figure 1. An example multiplex network showing the nonverbal social interaction (NVSI) variables in one-time layer (i.e., 30 minutes).

PRX in this time bracket was high over the network, resulting in high connectivity, and thus has been excluded for clarity of example. Edge weight represents the underlying variable value, node size represents multiplex centrality (i.e., the cumulative centrality score of a given node, when centrality across all NVSI layers is summed).

As for the subjective data, each participant rated the creative ties they experienced with other participants during the meetings as part of project debriefings (i.e., after every project meeting). We aggregated all creative dyadic mutual evaluations (DME) within a meeting as a network structure where the individual meeting participants are the nodes and their creative ties are directed and weighted edge sets connecting the nodes. 
Since most team members have filled out the post-meeting surveys, the DME networks per meeting are close to fully connected, whereas the NVSI as aggregated per session are not. We decided to set a threshold of 0.70 for the DME network edge sets, to capture only the highest bracket of creative activation. We dropped all DME dyads below this 70 percent threshold. We also treat these networks as simplex with unilateral edge sets. Thus, given any pair of nodes (dyad), creative activation exists where at least one meeting participant has evaluated the other with a score greater than o.7o. We then proceeded to make topological comparisons between the NVSI and DME networks by employing network alignment to produce evidence of the link between NVSI topology and 'creative activation'.

\subsection{Methodological Objectives}

We have two methodological objectives in this study; first, we produce a topological analysis of DME and NVSI networks to produce evidence of the link between NVSI topology and 'creative activation'. Second, we aim to isolate patterns in social signals (i.e. interaction synchrony) at different points in time that contribute most to this result. Here, we discuss our method comprising (1) network alignment, and, (2) network alignment evaluation with classification.

\subsection{Many-to-Many Pairwise Network Alignment}

Computational pairwise and multiple-network comparison of graph topology, and specifically network alignment, have been the subject of extensive research in discrete mathematics (Emmert-Streib, Dehmer \& Shi, 2016), especially in the context of computational biology and bioinformatics, where protein-protein interaction networks are commonly studied in this manner (Koyutürk, Kim, Topkara, et al., 2006). The strengths of the approach include the formalized and intuitive comparison of networks with common core features, but different topological structures. This approach also facilitates analysis of network dynamism (at granular and aggregate levels), which in our study, arises from the real-time nature of the data collection during meetings.

The dynamic many-to-many pairwise network alignment procedure developed in this study involves mapping networks computed from dyadic second-by-second level measurement, of DME and NVSI networks as a basis for identifying topologically-derived creative activation. The second-by-second social signal data is aggregated at 30 minute intervals to generate sufficient connectivity for network alignment. We then evaluate the network alignment with a predictive supervised machine learning classification exercise. This involves classifying the alignment patterns between different groupings of the DME networks and the NVSI networks to establish clear differentiation in the alignment results (i.e. between cases of high and low creative activation). This reinforces the evidence for creative activation deriving from the topology of the NVSI networks.

Alignments were performed using GraphCrunchz (Kuchaiev, Stevanović, Hayes, \& Pržulj, 2011). We used four measures, namely (1) graphlet degree distribution (GDD), (2) relative graph frequency distance (RGF), (3) path difference, and (4) clustering difference. Firstly, graphlets are clique-like subset of vertices of a network, described in Pržulj (2007). Graphlet degree distribution is a local-level topological comparison based on the graphlet degree distribution of focal networks (Kuchaiev et al., 2011; Pržulj, 2007). The measure ranges 0-1, where 1 is the best fit (Kuchaiev et al., 2011). Secondly, relative graph frequency distance 
(RGF) is a measure comparing the frequency with which graphlets appear between two networks, with the aim being to minimize this value (e.g. a value closer to zero equals greater similarity) (Kuchaiev et al., 2011). Thirdly, path difference measures the average path distance through the network (Albert \& Barabási, 2002; Yang et al., 2016). Lastly, clustering difference is based on the difference between clustering coefficients, capturing the tendency of nodes to cluster together, especially as network size increases (Albert \& Barabási, 2002; Kuchaiev et al., 2011). For both, higher scores equal more difference. GraphCrunch2 works with simplex networks, and thus automatically reduces bilateral edge sets to unilateral edge sets, and removes self-loops (i.e. for the variable TTK) (Kuchaiev et al., 2011).

\subsection{Creative Activation}

As mentioned in the variables' operationalization section (3.4.), we only consider the highest bracket of DME (i.e., above the 70 percent threshold) as simplex unilateral edge sets. The dynamic many-to-many pairwise network alignment procedure maps the DME and NVSI networks as a basis for identifying topologically-derived creative activation (in the upper $70 \%$ creativity bracket). The activation occurs when any graphlet of NVSI matches the creativity graphlet. If the match is high, then we call that high creative activation (HCA). If the match is low, then we have identified a case of low creative activation (LCA). We exemplify one such alignment within the Same NVSI and Same Meeting in Appendix 3 (A and B).

\section{Results}

The network alignment procedure identified 4212 alignments for TM $(\mathrm{HCA}=1404$, LCA = $2808)$, and 3456 alignments for TG $(\mathrm{HCA}=1152$, LCA $=2304)$. The breakdown of raw alignments is presented in Appendix 4. We explored the alignments between graphlets in the meeting corresponding the DME (i.e., DME Same) to identify matching patterns within every meeting. We were also interested in detecting the same patterns occurring in other topologies in either other meeting (see 'by meeting' plots) or within other NVSI (see 'by variable' plots).

\subsection{Descriptives}

In this section we present basic descriptives for each team by alignment metric. To identify topological evidence of creative activation we ran a simple comparison of the results for alignment quality between DME matrices and NVSI networks. To do this, we took the GDD measure of all alignments, and filtered this to GDD >0.70 (indicating a high level of similarity between any two given networks). The breakdown of alignments' metrics is presented in Appendix 4. The comparisons shown in Figures 2 to 5 show 'matches' between DME and NVSI networks based on this criterion, where a match is a true or false value, and the plots show an absolute count of these.

\subsubsection{Alignments 'By Meeting'}

The 'by meeting' plots (Figures 2 and 3) show a count of matches within specific meetings (i.e. the number of timeframe aggregates and matches by NVSI variable where a 'true' value was counted). Separate lines represent the total count (all DME matrices aligned to all NVSI networks), "DME Same" where the DME matrix and NVSI network belong to the same meeting (i.e. a DME matrix from Meeting $x$, aligned to an NVSI network of any NVSI variable 
from Meeting $x$ ), and "DME Other", where the DME matrix and NVSI network belong to different meetings (i.e. a DME matrix from Meeting $x$, aligned to an NVSI network of any NVSI variable from Meeting $y$ ).

The figures below are plotted with all alignment counts normalized to a one-to-zero scale to show relative values in the figures. DME Same HCA are represented in blue, DME Other HCA are depicted in orange, DME Same LCA are illustrated in green, and DME Other LCA are shown in red.

For TM, Figure 2 shows that creative activation for "HCA Same" was highest in meetings three and eight. This figure also shows that total matches increased sharply from meeting six onward. The "DME Other" for both HCA and LCA show greater topological similarity between all networks in the later meetings.

For TG, Figure 3 shows that creative activation for "HCA Same" was highest in meetings one, two, and to some extent three; thereafter it dropped off. "LCA Same" exhibits very high level of matches within the same meetings in M6 and M7. The total matches were highest for meeting seven.

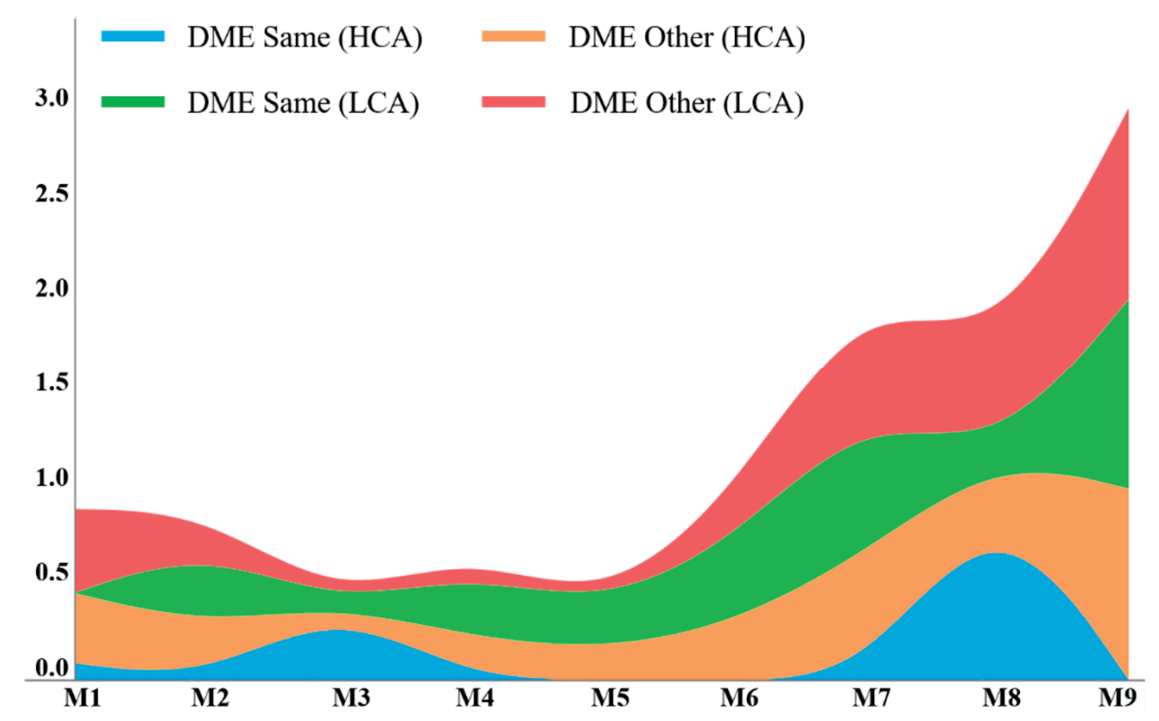

Figure 2. TM - Creative Activations by Meeting 


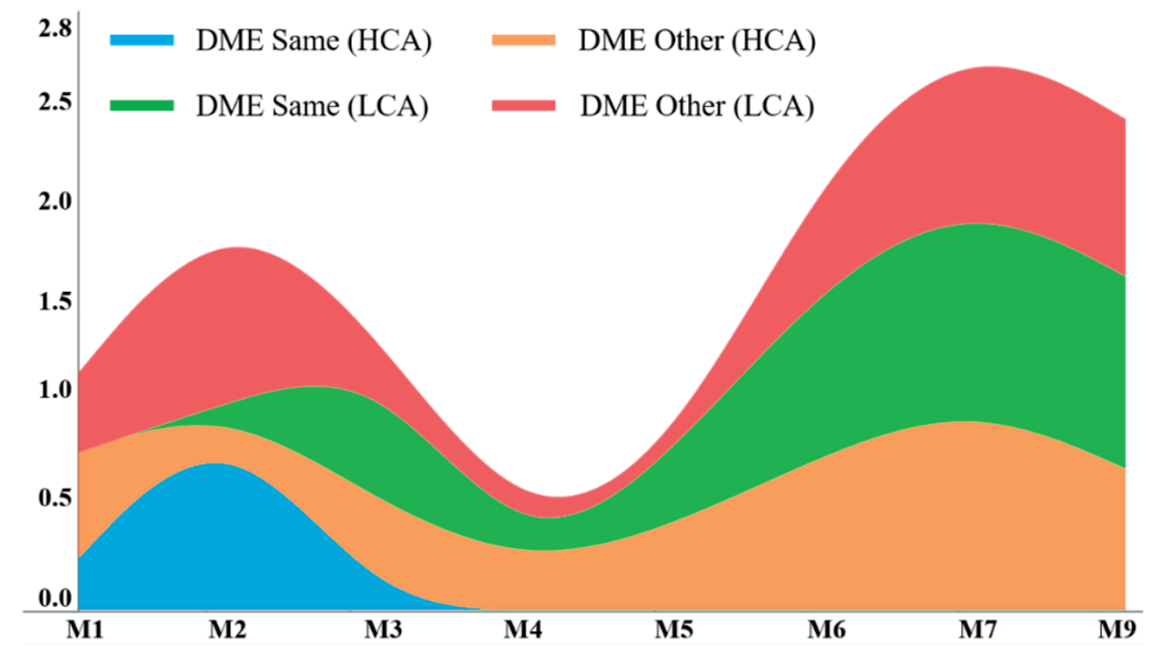

Figure 3. TG - Creative Activations by Meeting

\subsubsection{Alignments 'By Variable'}

Figures 4 and 5 show where these counts are derived from (i.e. based on NVSI variables). For TM, Figure 4 shows that the variables with the highest levels of matches with DME were $F_{2} F$ and PRX. One possible explanation was that those team members who faced each other or were in proximity evaluated their creative tie proportional and topologically similar with the time spent in close proximity. The next highest "DME HCA Same" was TTK.

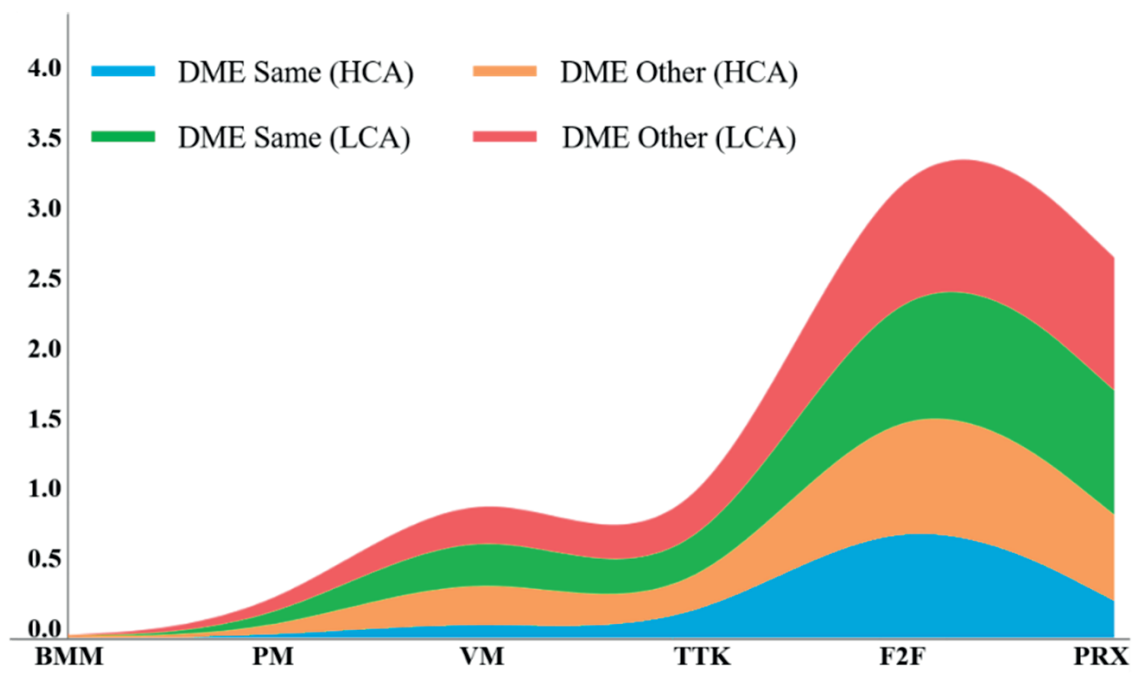

Figure 4. TM - Creative Activations by Variable

As for TG, Figure 5 below shows a similar matching trend as that of TM in Figure 4. The main difference between the two is that, in the case of TG, matches for HCA (same meeting) were 
relatively higher than TM across all variables. The $\mathrm{F}_{2} \mathrm{~F}$ variable has again the greatest count which is an indication that, as in the case of TM, the highest creativity dyads have mostly been reported by team members standing next to the other team members.

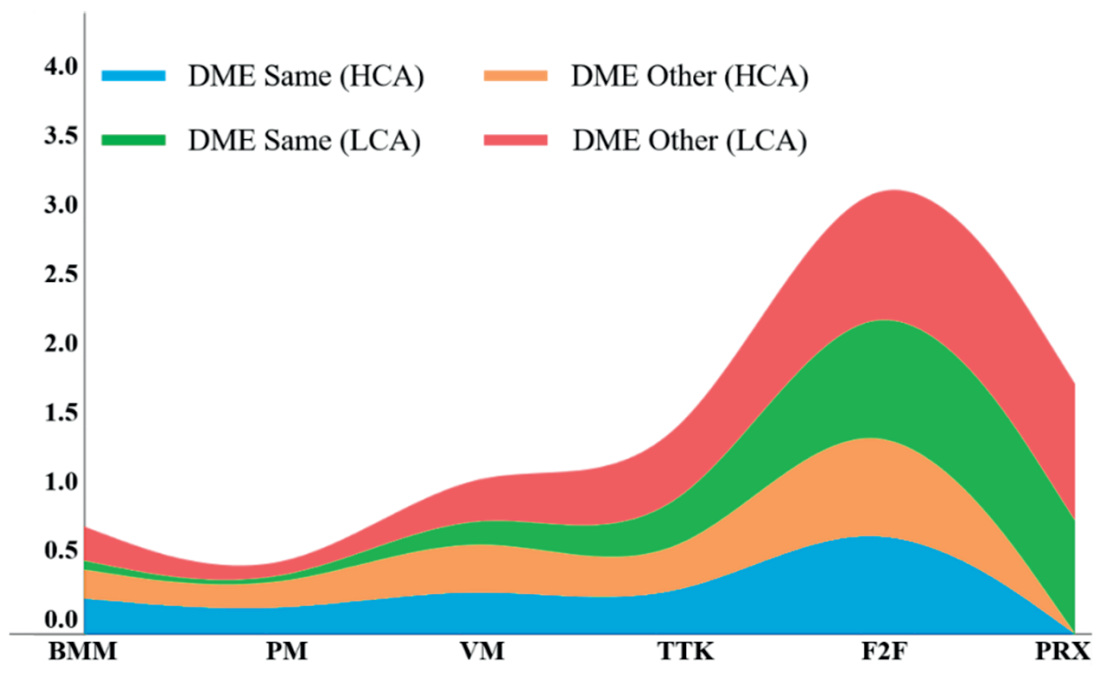

Figure 5. TG - Creative Activations by Variable

If we corroborate the 'by meeting' and 'by NVSI' figures we gain a clear example of what contributed most to HCA Same. For example, given Figures 2 and 4, Meeting 9 in TM scored the highest number of matches, and these derived primarily from the variable $F_{2} \mathrm{~F}$ and PRX. This pattern was similar for TG with TTK also scoring relatively high (Figures 3 and 5).

\subsection{Network Alignment Evaluation}

We evaluate the network alignment results using the REP Tree classifier from the WEKA Machine Learning Library (WEKA; 2017a), applied as multi-class classifiers (WEKA; 2017b). We decided for this algorithm as it is a fast decision tree learner. The mechanics involve building a decision tree by (1) sorting values for numeric attributes once and then (2) by using the variance. It prunes the decision tree by using a reduced-error pruning method with backfitting (WEKA; 2017c). We run this decision tree classifier using the no pruning parameter enabled and disabled (shown as [False] and [True]). Preceding this, we balanced the classes using the SMOTE oversampling procedure (described in Chawla et al., 2002). We thus ran two classifiers for each team (M and $G$ ). The target class labels were "HCA Same", "HCA Other", "LCA same" and "LCA Other".

Thus the classification problem becomes: 'Can we show topological evidence of creative activation in alignments between the most connected DME networks (where minimum edge weight is 0.70 ) and the NVSI networks?'

The results of the classifiers are summarized in Tables 1 and 2. 
Table 1. Network Alignment Evaluation Results for TM

\begin{tabular}{l|l|c|c|c|c}
\multirow{2}{*}{ Classifier } & Class & Recall & Precision & $\begin{array}{c}\text { F- } \\
\text { measure }\end{array}$ & Accuracy \\
\hline \multirow{3}{*}{$\begin{array}{l}\text { REP TREE } \\
\text { NP[F] }\end{array}$} & HCA Same & 0.87 & 0.83 & 0.85 & \\
\cline { 2 - 5 } & LCA Same & 0.8 & 0.75 & 0.77 & \multirow{2}{*}{$\mathbf{7 9 . 5 1 \%}$} \\
& HCA Other & 0.82 & 0.82 & 0.82 & \\
\hline \multirow{3}{*}{ REP TREE } & LCA Other & 0.69 & 0.78 & 0.73 & \\
NP[T] & HCA Same & 0.86 & 0.88 & 0.87 & \\
\cline { 2 - 4 } & LCA Same & 0.78 & 0.79 & 0.79 & \multirow{2}{*}{$\mathbf{8 1 . 3 1 \%}$} \\
& HCA Other & 0.87 & 0.8 & 0.83 & \\
\hline \hline
\end{tabular}

Table 2. Network Alignment Evaluation Results for TG

\begin{tabular}{l|l|c|c|c|c}
\multirow{2}{*}{ Classifier } & Class & Recall & Precision & $\begin{array}{c}\text { F- } \\
\text { measure }\end{array}$ & Accuracy \\
\hline \multirow{3}{*}{$\begin{array}{l}\text { REP TREE } \\
\text { NP[F] }\end{array}$} & HCA Same & 0.82 & 0.84 & 0.83 & \\
\cline { 2 - 5 } & LCA Same & 0.8 & 0.81 & 0.8 & \multirow{2}{*}{$\mathbf{8 1 . 1 6 \%}$} \\
\cline { 2 - 5 } & HCA Other & 0.85 & 0.79 & 0.82 & \\
\hline \multirow{3}{*}{$\begin{array}{l}\text { REP TREE } \\
\text { NP[T] }\end{array}$} & LCA Other & 0.78 & 0.81 & 0.8 & \\
\cline { 2 - 5 } & HCA Same & 0.83 & 0.87 & 0.85 & \\
& HCA Other & 0.87 & 0.78 & 0.82 & \multirow{2}{*}{$\mathbf{8 2 . 9 3 \%}$} \\
\hline \hline
\end{tabular}

As shown in Tables 1 and 2, recall, precision and F-measure scores are strong and wellbalanced across all class labels, although the results are slightly better for the two HCA classes. Overall model accuracy is strong, ranging between $79.51 \%$ and $82.93 \%$. Tables 3 and 4 show the confusion matrices for each classifier.

The matrices show the overall strong performance of the classifier in terms of distinguishing between classes, but also where minimal misclassification did occur; this was between the two HCA classes, and the two LCA classes respectively. This could be due to there being a natural similarity between classes (e.g., the team members evaluated the creative ties consistently across meetings). 
Table 3. Confusion Matrices for Each Classifier for TM

\begin{tabular}{rl|c|c|c|c|c} 
& & HCA Same & HCA Other & LCA Other & LCA Same \\
REP TREE NP[F] & HCA Same & 2173 & 269 & 47 & 19 \\
& HCA Other & 324 & 2065 & 52 & 67 \\
LCA Other & 77 & 120 & 1741 & 570 \\
LCA Same & 43 & 69 & 399 & 1997 \\
REP TREE NP[T] & HCA Same & 2155 & 287 & 43 & 23 \\
& HCA Other & 203 & 2178 & 77 & 50 \\
& LCA Other & 53 & 146 & 1858 & 451 \\
& LCA Same & 33 & 127 & 382 & 1966
\end{tabular}

Table 4. Confusion Matrices for Each Classifier for TG

\begin{tabular}{rl|c|c||c|c} 
& & HCA Same & HCA Other & LCA Other & LCA Same \\
REP TREE NP[F] & HCA Same & 1694 & 313 & 39 & 24 \\
& HCA Other & 232 & 1759 & 42 & 37 \\
& LCA Other & 54 & 76 & 1621 & 319 \\
& LCA Same & 30 & 92 & 302 & 1646 \\
REP TREE NP[T] & HCA Same & 1713 & 303 & 40 & 14 \\
& HCA Other & 190 & 1791 & 47 & 42 \\
& LCA Other & 38 & 109 & 1699 & 224 \\
& LCA Same & 22 & 101 & 283 & 1664
\end{tabular}

Having looked at the over models' performance, we will now investigate the performance of the input features for the classifier. Table 6 in Appendix 4 provides a brief overview of the raw counts of alignment (1) 'by meeting' and (1) 'by variable' for both teams. (1) The highest count in the HCA Same class occurred for TM in Meeting 8 and for TG in Meeting 2. The most alignments in the LCA class were identified in Meeting 9 for TM and Meetings 7 and 8 for TG. (2) The highest count 'by variable' recorded in the HCA Same class was with the F2F variable for both teams; as for the LCA Same class, F2F and PRX scored the highest number of alignments in the case of both teams.

Tables 7 and 8 Appendix 4 present the alignment metrics of the input data, namely the means of the graphlet degree distributions (GDD), the relative graph frequency distances (RGF), path differences, and clustering differences.

Firstly, GDD is the results of topological comparisons between the graphlet degree distributions of DME and NVSI networks. All values are normalized o-1 for visualization, where 1 is the best fit. Figures 6 and 7 show that, for TM, the GDD scores are higher for the 
two LCA classes and lower for the two HCA classes. In other words, the local-level topological comparison (based on the graphlet degree distribution) of the NVSI networks have a better fit for LCA classes than with the two HCA classes. For TG, Figures 8 and 9 show that the GDD scores are higher for the two LCA classes, and lower for the two HCA classes. The input features that show the best fir for both TM and TG are PRX, F2F, and VM.

Secondly, RGF compared the frequency with which graphlets appear between two networks, whereby a value closer to zero equals greater similarity. As expected, path difference tended to be higher for the HCA classes. In other words, the average path distance is smaller through the LCA classes (that also had a better fit) than in the HCA classes. The RGF results for TG consistent with the observations from TM.

Thirdly, path difference measured the average path distance through the network. For TM, path difference tended to be higher for the HCA classes. In other words, the average path distance is smaller through the LCA classes (that also had a better fit) than in the HCA classes. For TG, path difference is mixed across classes.

Lastly, clustering difference calculated the difference between clustering coefficients, thus showing the tendency of nodes to cluster together. For TM, the cluster difference is noticeably relatively higher among the variables in all four classes. In other words, across all classes there is a tendency of nodes to cluster together. For TG, the cluster difference scores appear higher for the two LCA classes. For both TM and TG, the variable PRX has relatively higher scores.

\section{Discussion}

In brief, in this study we have presented topological evidence of creative activation as a result of alignments between the NVSI networks and the most connected and highest valued DME networks. We validated our results using a novel classification algorithm which produced a strong accuracy for both teams as the NVSI were accurately classified to the high and low creativity targets. We thus confirm our prediction stated in RQ1 on topological social network convergence. We also extended our results to include other meetings in the available dataset, as a proxy for more generalizable insights.

The topological analysis of DME and NVSI networks to produced evidence of the link between NVSI topology and 'creative activation', and isolated patterns in the social signals (i.e. interaction synchrony) at different points in time that contribute most to this result. Our network alignment exercise shows meaningful differences when creative activation is most prevalent in a sequence of meetings, and from which variables this derives, namely the time spent in proximity and face-to-face, and mirroring of voice volume. This answered our second $R Q$.

\subsection{Methodological Contributions}

This study provides two main methodological contributions. On the one hand, we employ wearable teach to collect objective data on social interactions and we combine it with subjective measurements. On the other hand, we introduce network alignment as a new methodology to social sciences that is deep-rooted in discrete mathematics (Emmert-Streib et al., 2016), especially in the fields of computational biology and bioinformatics (Koyutürk 
et al., 2006). The strengths of the approach include the formalized and intuitive comparison of networks with common core features, but different topological structure. This approach also facilitates analysis of network dynamism, which in our study, arises from the real-time nature of the data collection during meetings.

\subsection{Theoretical Contributions}

Previous literature investigated several antecedents of team creativity such as team composition (Perry-Smith \& Shalley, 2014), task definition (Gilson \& Shalley, 2004), and team interactions (Sosa, 2011; Sosa et al., 2015). In the tangent and well-established research stream of team performance nonverbal social interactions have been shown be relevant predictors of collaboration quality and team performance (de Montjoye et al., 2014; Pentland, 2012; Shmueli, et al., 2014; Woolley et al., 2010).

We contribute to the existing literature by answering calls for team research on objective measurements of social signals with high-frequency sampling rates (Kozlowski, 2015) by deploying the Sociometric badges as the core data collection tool. We also address the calls for temporal precedence in team research (de Montjoye et al., 2014; Kozlowski, 2015) by conducting two longitudinal field studies enabled by data collection with wearable technology.

Our findings reflect that the highest evaluated creativity dyads have been reported by team members standing next to the other the most. This finding has three potential explanations. Firstly, team members might be actively seeking to spend the most time in the immediate vicinity of those who they perceive having a creative tie with; in this case, the perceived creative tie would preexist and influence physical distance. Secondly, it is possible that team members are more capable to evaluate their creative interaction with those with which they collaborated longer. Thirdly, given that the team meetings were supposedly task-focused, the time spent face-to-face could have been augmented by the assignment of expertisespecific sub-teams. The members of these sub-teams could conceivably have evaluated their creative ties highly due to the sub-team homophily. This would be consistent with social network research, whereby individuals are more likely to interact with others who share some of their attributes (McPherson, Smith-Lovin, \& Cook, 2001).

While the overall proxemics descriptives are quite intuitive, by contrast, the best topology matches (i.e., best model fit) occur for the low creative activation classes. Considering that the low classes have been defined for the upper 30 percent creative ties, the results express that the nonverbal behavior is best synchronized with second highest creativity ties.

Both teams exhibit ascending trends of matches over time, derived primarily from the time spent face-to-face, the time spent in proximity, and the mirroring of voice volume. Therefore, the creation and usage of communication channels (i.e., turn-taking) does not exhibit significant alignment with the creative ties, but, contrarily, the way the team members communicated (i.e. the volume mirroring of their voices) sparks the creative activation. This finding specifically contributes to the fundamental work of Bernieri and Rosenthal (1991) on behavioral convergence by adding that the nonverbal behavior (i.e., social signals) converges with the verbal behavior (i.e., creative problem solving) acting as a new valid proxy for consensus. 


\subsection{Practical Implications}

Previous research suggests that SMEs in low-tech B2B industries are keenly considering involving customers in their development processes (Chang \& Taylor, 2016). As customer participation gains traction (Cui \& Wu, 2015), many firms are likely to jump on the bandwagon without assessing how the process will unfold. Our study investigates customer involvement in new service development. The two B2B co-developing teams in our sample were monitored over the course of their entire project spans. The results deepen the managerial understanding of creativity implementation in projects from a human resource management perspective, and offer guidance to companies hoping to engage in customer involvement.

The first key observation was that the multi-disciplinary multi-stakeholder teams did not exhibit creative activations in the early stages of the development process. Managers should therefore allow the team members time to synchronize their ideas and behaviors to successfully carry out the project.

Aside for the time dimension, our results suggest that meetings that require team members to spend more time face-to-face and in each other's proximity are more likely to bring about creative relationships. One behavioral indication of the interactional synchronicity is the mirroring in voice volume.

\subsection{Limitations}

This study has some limitations that future research can address. First, we do not identify key team members, dyads, or cliques that foster or hinder the creative activation, as we only investigate the overall topology of our focus variables. Investigating cliques that are bound to bring activate creativity would be particularly interesting as it could add to the growing body of literature on the social side of creativity, where every position in the network provides a different perspective on the interactions and has its advantages and disadvantages.

Second, future research should investigate the interplay between the customer and firm by identifying and isolating dyads of customer-to-customer, customer-to-firm, and firm-tofirm.

Third, we deduced the connectivity thresholds empirically; future research should test for optimal cut-off values in their variables' operationalization. Fourth, as we did not have access to the actual conversational content due to confidentiality issues, we were unable to fully explain what occurred in the meetings that led to greater topological similarity between all networks in the later meetings or to higher levels of matching for high creative activations within the same meeting or to the creative activations to drop completely. Future research should investigate the symbiosis between the verbal and the nonverbal behavior drawn from solely objective measures.

\section{5. $\quad$ Future Research Directions}

Future quantified employee research will incorporate additional ways of measuring focal dependent variables such as creativity. Although self-report methods at the end of meetings best mirror the format of an in-house company meeting (i.e. with an end of project debriefing session and reporting exercise), future research will draw upon developments in dynamic 
data collection for social science, such as ecological momentary assessment (see: Spanakis, Weiss, \& Roefs, 2016) and will also incorporate physiological measures that correspond with manifestations of focal dependent variables (Kandasamy, Hardy, Page et al., 2014; Villarejo, Zapirain, \& Zorrilla, 2012). In line with this, future research will also compare time series techniques against aggregated network analysis approaches (Lee, Rowlands, Jackson, Leadbetter, Wada, \& James, 2017; Shameer, Badgeley, Miotto, \& et al., 2016).

Conceptually, future research will explore the implications of NVSI for incentive system design (Grabner, 2014; Li, Deng, Leung, \& Zhao, 2017), as well as broader implications for workplace culture and format (Dul, Ceylan, \& Jaspers, 2011), knowledge sharing (Carmeli, Gelbard, \& Reiter-Palmon, 2013) and ambidextrous organizational learning (Prieto \& Pilar Pérez Santana, 2012), along the lines of comparing employee identification with the project teams versus the organization (Lin, He, Baruch, \& Ashforth, 2016; Liu, Keller, \& Shih, 2011; Olsen, Sverdrup, Nesheim, \& Kallenberg, 2016), contrasted with customer co-development (Cui \& Wu 2015). 


\section{Appendix 1. The split of sessions across project meetings across teams}

Table 5. Project meetings and sessions for TM and TG.

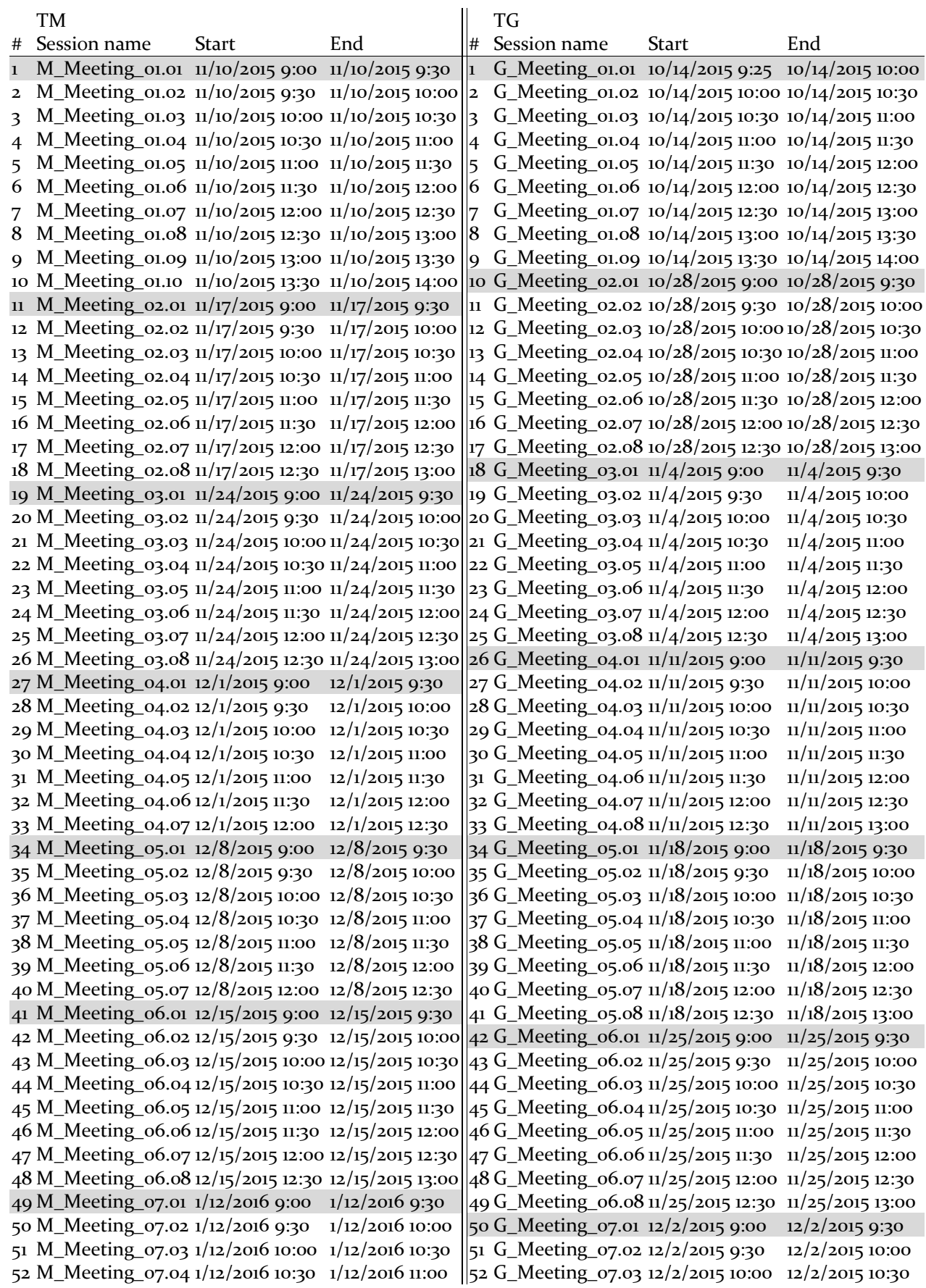


53 M_Meeting_07.05 1/12/2016 11:00 1/12/2016 11:30 54 M_Meeting_07.06 1/12/2016 11:30 1/12/2016 12:00 55 M_Meeting_07.07 1/12/2016 12:00 1/12/2016 12:30 56 M_Meeting_07.08 1/12/2016 12:30 1/12/2016 13:00 57 M_Meeting_07.091/12/2016 13:00 1/12/2016 13:30 58 M_Meeting_07.10 1/12/2016 13:30 1/12/2016 14:00 59 M_Meeting_o8.01 1/19/2016 9:00 1/19/2016 9:30 6o M_Meeting_o8.02 1/19/2016 9:30 1/19/2016 10:00 61 M_Meeting_o8.03 1/19/2016 10:00 1/19/2016 10:30 62 M_Meeting_08.04 1/19/2016 10:30 1/19/2016 11:00 63 M_Meeting_o8.05 1/19/2016 11:00 1/19/2016 11:30 64 M_Meeting_o8.06 1/19/2016 11:30 1/19/2016 12:00 65 M_Meeting_o8.07 1/19/2016 12:00 1/19/2016 12:30 66 M_Meeting_o8.08 1/19/2016 12:30 1/19/2016 13:00 67 M_Meeting_08.091/19/2016 13:00 1/19/2016 13:30 68 M_Meeting_o8.10 1/19/2016 13:30 1/19/2016 14:00 69 M_Meeting_09.01 1/26/2016 9:00 1/26/2016 9:30 70 M_Meeting_09.02 1/26/2016 9:30 1/26/2016 10:00 71 M_Meeting_o9.03 1/26/2016 10:00 1/26/2016 10:30 72 M_Meeting_o9.041/26/2016 10:30 1/26/2016 11:00 73 M_Meeting_09.05 1/26/2016 11:00 1/26/2016 11:30 74 M_Meeting_o9.061/26/2016 11:30 1/26/2016 12:00 75 M_Meeting_09.07 1/26/2016 12:00 1/26/2016 12:30 76 M_Meeting_o9.08 1/26/2016 12:30 1/26/2016 13:00 77 M_Meeting_o9.091/26/2016 13:00 1/26/2016 13:30 78 M_Meeting_o9.10 1/26/2016 13:30 1/26/2016 14:00
53 G_Meeting_07.04 12/2/2015 10:30 12/2/2015 11:00 54 G_Meeting_07.05 12/2/2015 11:00 12/2/2015 11:30 55 G_Meeting_07.06 12/2/2015 11:30 12/2/2015 12:00 56 G_Meeting_07.07 12/2/2015 12:00 12/2/2015 12:30 57 G_Meeting_07.08 12/2/2015 12:30 12/2/2015 13:00 58 G_Meeting_08.01 1/20/2016 9:00 1/20/2016 9:30 59 G_Meeting_08.02 1/20/2016 9:30 1/20/2016 10:00 6o G_Meeting_08.03 1/20/2016 10:00 1/20/2016 10:30 61 G_Meeting_08.04 1/20/2016 10:30 1/20/2016 11:00 62 G_Meeting_08.05 1/20/2016 11:00 1/20/2016 11:30 63 G_Meeting_08.06 1/20/2016 11:30 1/20/2016 12:00 64G_Meeting_08.071/20/2016 12:00 1/20/2016 12:30

\section{Appendix 2. Stabilization coefficient versus mean for calculating the mirroring ties}

Example of posture mirroring [PM] data collected off two members of Team M in meeting 2, session 7 exported as minute-by-minute-split datasets per session marking the instances when mirroring took place on a scale from -1 to 1 .

In the example below, the mirroring variation over the 30 minutes is marginal overall but they hint at a slightly more positive tendency (the SC of posture mirroring equal 0.008 and 0.013 respectively). In other words, in the 30 minutes aggregated below node "B" has an overall slightly positive reciprocated mirroring tie with node "A". If we consider the distinct means instead of the SC the interaction seems quite unbalanced as the mirroring scores are inflated (as the mean scores of posture mirroring equal 0.037 and 0.077 respectively).

$$
\begin{gathered}
P M_{\text {neg }}=\overline{P M} \text {, if } P M \in[-1,0) \text { and } P M_{\text {pos }}=\overline{P M}, \text { if } P M \in(0,1] \\
S C=\frac{\left(P M_{\text {neg }}+P M_{\text {pos }}\right) / 2}{\left|P M_{\text {pos }}-P M_{\text {neg }}\right| / \sqrt{2}}
\end{gathered}
$$


Table 6: The differences between the mean and the SC, and the resulting aggregated variation of PM in the same dyad

\begin{tabular}{|c|c|c|c|}
\hline & & $\mathbf{A}$ & B \\
\hline & Timestamp & B & A \\
\hline & 11/17/15 12:00 & o & 0.797944 \\
\hline & 11/17/15 12:01 & $\mathrm{o}$ & o \\
\hline & 11/17/15 12:02 & 0.643178052 & o \\
\hline & 11/17/15 12:03 & o & o \\
\hline & 11/17/15 12:04 & o & o \\
\hline & 11/17/15 12:05 & 0.854664203 & 0.854664 \\
\hline & 11/17/15 12:06 & o & o \\
\hline & 11/17/15 12:07 & o & -0.64428 \\
\hline & 11/17/15 12:12 & -0.661057759 & -0.66106 \\
\hline & 11/17/15 12:13 & o & o \\
\hline & 11/17/15 12:17 & 0.670982865 & 0.670983 \\
\hline & 11/17/15 12:18 & o & o \\
\hline & 11/17/15 12:19 & o & -0.82225 \\
\hline & $11 / 17 / 1512: 20$ & o & 0.712738 \\
\hline & 11/17/15 12:21 & -0.698096836 & o \\
\hline & 11/17/15 12:22 & o & o \\
\hline & $11 / 17 / 1512: 23$ & 0.634506331 & o \\
\hline & 11/17/15 12:24 & -0.693309077 & o \\
\hline & $11 / 17 / 1512: 25$ & o & o \\
\hline & 11/17/15 12:28 & $\mathrm{o}$ & 0.650931 \\
\hline \multirow[t]{5}{*}{ Mean: } & $=\overline{P M}$ & 0.037543 & 0.077984 \\
\hline & $P M_{\text {neg }}$ & -0.684154557 & -0.709196667 \\
\hline & $P M_{\text {pos }}$ & 0.700832863 & 0.737452 \\
\hline & $\left(P M_{\text {neg }}+P M_{\text {pos }}\right) / 2$ & 0.008339153 & 0.014127667 \\
\hline & $\left|P M_{\text {pos }}-P M_{\text {neg }}\right| / \sqrt{2}$ & 0.979333997 & 1.022935082 \\
\hline \multirow{2}{*}{$\begin{array}{c}\text { Stabilization } \\
\text { coefficient: }\end{array}$} & $=\frac{\left(P M_{\text {neg }}+P M_{\text {pos }}\right) / 2}{2}$ & \multirow{2}{*}{0.008515} & \multirow{2}{*}{0.013811} \\
\hline & $=\overline{\left|P M_{\text {pos }}-P M_{\text {neg }}\right| / \sqrt{2}}$ & & \\
\hline
\end{tabular}

Resulting aggregated "PM mean" dyad:

\begin{tabular}{|c|c|c|}
\cline { 2 - 3 } \multicolumn{1}{c|}{} & A & B \\
\hline A & o & 0.077984 \\
\hline B & 0.037543 & 0 \\
\hline
\end{tabular}

Resulting aggregated "PM SC" dyad:

\begin{tabular}{|c|c|c|}
\cline { 2 - 3 } \multicolumn{1}{c|}{} & A & B \\
\hline A & 0 & 0.013811 \\
\hline B & 0.008515 & 0 \\
\hline
\end{tabular}




\section{Appendix 3.}

A. Example of alignment from Meeting 1 of a Proximity graphlet from Session 1 with the target variable (i.e., the Creativity graphlet of Meeting 1)

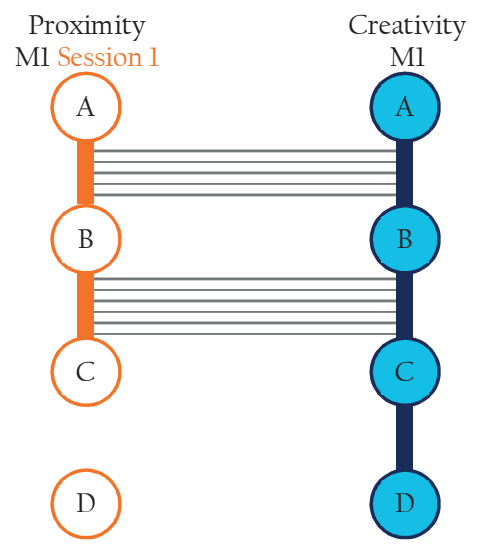

B. Examples of High-Low Same-Other creative activation in two graphlets

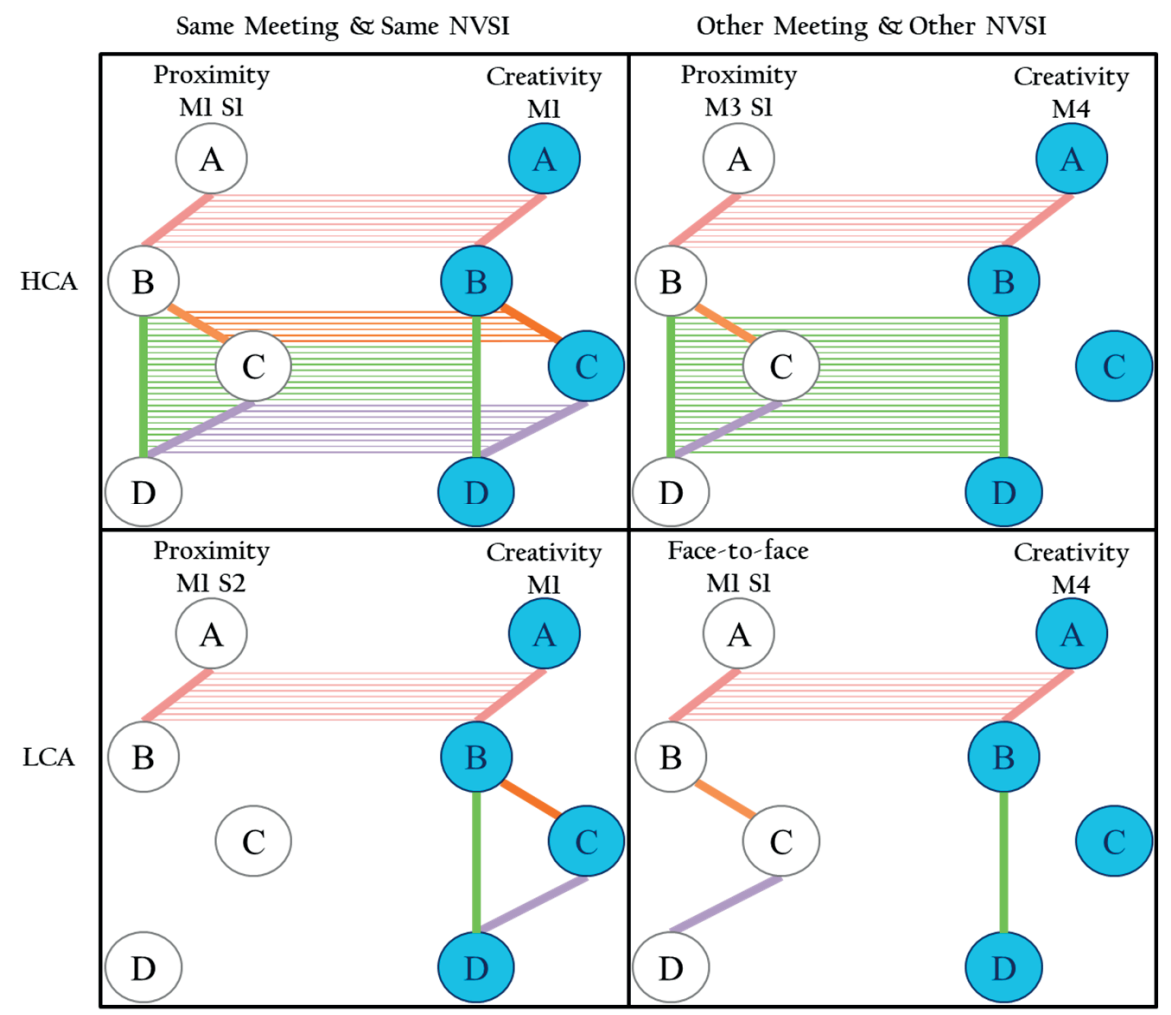


Appendix 4. Overview of the input data for the classifier, showing averages for each input feature (Table 6) and alignment metrics (Tables 7 and 8 ).

Table 6. Raw counts for alignments 'by meeting' and 'by variable' for TM and TG

\begin{tabular}{|c|c|c|c|c|c|c|c|c|}
\hline & \multicolumn{2}{|c|}{ DME Same (HCA) } & \multicolumn{2}{|c|}{ DME Same (LCA) } & \multicolumn{2}{|c|}{ DME Other (HCA) } & \multicolumn{2}{|c|}{ DME Other (LCA) } \\
\hline & TM & TG & $\mathrm{TM}$ & TG & $\mathrm{TM}$ & TG & TM & TG \\
\hline Meeting 1 & 2 & 4 & o & o & 48 & 13 & 174 & 161 \\
\hline Meeting 2 & o & 17 & 24 & o & 39 & o & 119 & 226 \\
\hline Meeting 3 & 9 & o & 0 & 31 & 7 & 13 & 78 & 138 \\
\hline Meeting 4 & o & o & 20 & o & 32 & 5 & 101 & 136 \\
\hline Meeting 5 & o & o & 14 & 16 & 24 & 10 & 75 & 122 \\
\hline Meeting 6 & o & o & 26 & 38 & 39 & 19 & 128 & 181 \\
\hline Meeting 7 & o & o & 45 & 45 & 81 & 25 & 224 & 205 \\
\hline Meeting 8 & 23 & o & o & 42 & 26 & 17 & 193 & 201 \\
\hline Meeting 9 & o & $N A$ & 60 & $N A$ & 119 & $N A$ & 300 & $N A$ \\
\hline BMM & 2 & 3 & 20 & 24 & 39 & 12 & 118 & 192 \\
\hline PM & 2 & 2 & 21 & 23 & 38 & 8 & 134 & 158 \\
\hline VM & 4 & 4 & 32 & 26 & 75 & 23 & 215 & 206 \\
\hline TTK & 2 & 2 & 22 & 26 & 39 & 8 & 147 & 218 \\
\hline $\mathrm{F}_{2} \mathrm{~F}$ & 18 & 9 & 49 & 38 & 131 & 48 & 401 & 301 \\
\hline PRX & 6 & 1 & 45 & 35 & 93 & 3 & 377 & 295 \\
\hline
\end{tabular}

Table 7. Alignment metrics for TM

\begin{tabular}{lccccc} 
Class & NVSI & $\begin{array}{c}\text { Mean } \\
\text { GDD }\end{array}$ & $\begin{array}{c}\text { Mean } \\
\text { RGF Distance }\end{array}$ & $\begin{array}{c}\text { Mean } \\
\text { Path Difference }\end{array}$ & $\begin{array}{c}\text { Mean } \\
\text { Cluster } \\
\text { Difference }\end{array}$ \\
\hline HCA - Same & BMM & 0.55 & 8.40 & 0.67 & 0.31 \\
& F2F & 0.72 & 3.51 & 1.06 & 0.31 \\
& PM & 0.56 & 8.48 & 0.69 & 0.33 \\
& PRX & 0.64 & 7.57 & 1.01 & 0.56 \\
& TTK & 0.56 & 8.15 & 0.72 & 0.35 \\
& VM & 0.60 & 5.76 & 0.58 & 0.28 \\
\hline HCA - Other & BMM & 0.59 & 6.98 & 0.72 & 0.33 \\
& F2F & 0.75 & 3.40 & 1.19 & 0.29 \\
& PM & 0.58 & 6.94 & 0.73 & 0.33 \\
& PRX & 0.67 & 6.50 & 1.05 & 0.56 \\
& TTK & 0.59 & 6.79 & 0.76 & 0.32 \\
& VM & 0.65 & 4.46 & 0.70 & 0.24 \\
\hline LCA - Same & BMM & 0.70 & 5.26 & 0.66 & 0.52 \\
& F2F & 0.96 & 0.46 & 0.47 & 0.10 \\
& PM & 0.70 & 5.28 & 0.67 & 0.52 \\
& PRX & 0.88 & 2.90 & 0.61 & 0.81 \\
& TTK & 0.70 & 5.09 & 0.64 & 0.54 \\
& VM & 0.79 & 3.05 & 0.64 & 0.29 \\
\hline LCA - Other & BMM & 0.68 & 6.72 & 0.68 & 0.53 \\
& F2F & 0.93 & 0.93 & 0.81 & 0.11 \\
& PM & 0.67 & 6.76 & 0.68 & 0.55 \\
& PRX & 0.85 & 3.85 & 0.59 & 0.82 \\
& TTK & 0.68 & 6.29 & 0.66 & 0.55 \\
& VM & 0.75 & 3.66 & 0.83 & 0.32 \\
\hline \hline
\end{tabular}


Table 8. Alignment metrics for TG

\begin{tabular}{lccccc} 
Class & NVSI & $\begin{array}{c}\text { Mean } \\
\text { GDD }\end{array}$ & $\begin{array}{c}\text { Mean } \\
\text { RGF Distance }\end{array}$ & $\begin{array}{c}\text { Mean } \\
\text { Path Difference }\end{array}$ & $\begin{array}{c}\text { Mean } \\
\text { Cluster } \\
\text { Difference }\end{array}$ \\
\hline HCA - Same & BMM & 0.56 & 6.94 & 0.79 & 0.35 \\
& F2F & 0.61 & 4.49 & 1.00 & 0.28 \\
& PM & 0.52 & 6.95 & 0.62 & 0.26 \\
& PRX & 0.61 & 7.44 & 0.86 & 0.44 \\
& TTK & 0.55 & 7.28 & 0.87 & 0.39 \\
& VM & 0.55 & 6.62 & 0.90 & 0.31 \\
\hline HCA - Other & BMM & 0.56 & 6.29 & 0.77 & 0.33 \\
& F2F & 0.62 & 4.94 & 1.11 & 0.43 \\
& PM & 0.55 & 6.76 & 0.71 & 0.33 \\
& PRX & 0.59 & 7.42 & 0.87 & 0.42 \\
& TTK & 0.56 & 6.80 & 0.81 & 0.34 \\
& VM & 0.58 & 5.59 & 0.96 & 0.33 \\
\hline LCA - Same & BMM & 0.76 & 3.62 & 0.64 & 0.49 \\
& F2F & 0.89 & 1.13 & 1.07 & 0.14 \\
& PM & 0.75 & 3.95 & 0.69 & 0.49 \\
& PRX & 0.81 & 3.45 & 0.77 & 0.69 \\
& TTK & 0.77 & 3.77 & 0.69 & 0.57 \\
& VM & 0.79 & 2.81 & 0.97 & 0.27 \\
\hline LCA - Other & BMM & 0.73 & 4.07 & 0.74 & 0.57 \\
& F2F & 0.85 & 1.71 & 1.06 & 0.18 \\
& PM & 0.70 & 4.87 & 0.70 & 0.53 \\
& PRX & 0.82 & 3.61 & 0.82 & 0.74 \\
& TTK & 0.75 & 4.00 & 0.79 & 0.63 \\
& VM & 0.75 & 3.54 & 0.94 & 0.40 \\
\hline \hline
\end{tabular}


Chapter 5

Conclusion 


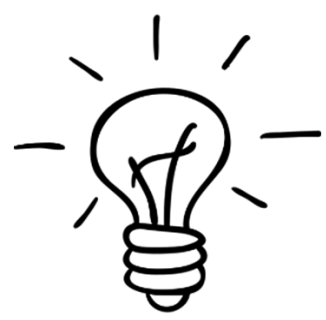

"I can't understand why people are frightened of new ideas. I'm frightened of the old ones."

- John Milton Cage Jr.

This dissertation built on a synthesis of the new service development, social creativity, and social signal processing literatures and their sub-branches. In this section, the outcomes and implications from the three constituent chapters of this dissertation are briefly summarized as follows. In the 'Overall Synopsis of Findings' we relate the problem statement in light of the design and aim of the empirical studies. In the 'Overall implications' we capture the overarching methodological contributions of this dissertation; in this section we also describe implications for theory and practice that are derived from our findings. Subsequently, we relate agenda items for future research. This chapter concludes with some final remarks.

\section{Synopsis of Findings}

As set out at the beginning of this dissertation, team literature should explain what kind of team interactions lead to certain types of creative outcomes. However, to date, most team research has focused on team effectiveness and teams' performance outcomes (Salas, Reyes, \& McDaniel, 2018; Salas, Shuffler, Thayer, Bedwell, \& Lazzara, 2015), with most extant studies employing the input-process-output model and assuming a linearly quantifiable outcome (i.e., a performance measure). As also introduced, most previous team research has centered on relatively limited cross-sectional designs and being heavily reliant on self-report data.

To address these issues, this dissertation focused on quantifying the objective behavior of teams and aimed to uncover how team creative mechanisms can be explained by objective and fine-grained behaviors in real-life new product or service development situations. The following section cover this in specific detail (as also summarized in Tables 1 and 2).

The results of the MRQAP procedure Chapter 3 showed that the relative time spent in positive posture mirroring decreases the alter-perceived creativity. In other words, if team members exhibit posture mirroring in most of the meeting's duration the perceived creativity decreases. In the penultimate meeting within this study, the time spent in face-to-face enhances the creative assessment of team members. This finding does not hold for the dyadic level where the face-to-face time had no impact on creativity.

At dyadic level, however, we found that turn-taking and proximity alone contributing an average of $29.87 \%$ improvement to models, with an average accuracy of $74.40 \%$ across meetings. In the last meeting within this study, we showed that turn-taking has significantly 
strong negative effect in this project phase. As discussed, in what is supposed to be the converging stage; the communication distribution negatively influences creativity.

Table 1. Overview of results for Chapter 3

Research question: How do social signals drive reciprocal creativity?

\begin{tabular}{|c|c|c|c|c|c|}
\hline & & MR & AP & Multi-Clas & ssification \\
\hline & & $\begin{array}{l}\text { Regression } \\
\text { Coefficient }\end{array}$ & Adj. $\mathbf{R}^{2}$ & $\begin{array}{c}\text { FFS } \\
\text { (Change) }\end{array}$ & $\begin{array}{c}\text { Model } \\
\text { Accuracy }\end{array}$ \\
\hline & $\begin{array}{l}\text { Meeting 1: } \\
\text { Exploration } \\
\text { and ideation }\end{array}$ & $P M=-0.266^{* *}$ & $\mathrm{R}^{2}=0.085^{* *}$ & $\begin{aligned} \mathrm{PRX} & =38.43 \% \\
\mathrm{TTK} & =\mathbf{2 2} .32 \% \\
\mathrm{PM} & =9.43 \%\end{aligned}$ & $76.33 \%$ \\
\hline Results & $\begin{array}{l}\text { Meeting 2: } \\
\text { Prototyping } \\
\text { the idea }\end{array}$ & $\mathrm{F}_{2} \mathrm{~F}=0.129^{*}$ & $\mathrm{R}^{2}=0.029^{* *}$ & $\begin{array}{l}\mathrm{TTK}=35.54 \% \\
\mathrm{PRX}=16.04 \% \\
\mathrm{BMM}=7.38 \%\end{array}$ & $64.04 \%$ \\
\hline & $\begin{array}{l}\text { Meeting 3: } \\
\text { Pitch of final } \\
\text { prototype }\end{array}$ & $\begin{array}{l}\text { TTK }=-0.506^{*} \\
\text { Note: }{ }^{* *} p<0.0 \\
\text { o.1 }\end{array}$ & $\begin{array}{c}\mathrm{R}^{2}=0.208^{* *} \\
{ }^{* *} p<0.05 ;{ }^{*} p<\end{array}$ & $\begin{array}{c}\mathrm{TTK}=45.79 \% \\
\mathrm{PRX}=\mathbf{2 1 . 1 0} \% \\
\text { Layer }=8.87 \%\end{array}$ & $81.67 \%$ \\
\hline $\begin{array}{l}\text { Key } \\
\text { Findings }\end{array}$ & $\begin{array}{l}\text { At team level } \\
\text { marginally in } \\
\text { to-face in me } \\
\text { the time sp } \\
\text { meeting } 1 \text { and }\end{array}$ & $\begin{array}{l}\text { 1, that the social } \\
\text { aproving (i.e., the } \\
\text { eting 2) or inhibit } \\
\text { ent in posture } \\
\text { turn-taking in } \mathrm{m}\end{array}$ & $\begin{array}{l}\text { gnals are either } \\
\text { ime spent face- } \\
\text { g creativity (i.e., } \\
\text { hirroring in in } \\
\text { eting 3). }\end{array}$ & $\begin{array}{l}\text { At dyadic level, } \\
\text { taking and proxi } \\
\text { an average of } 2 \\
\text { models predicti } \\
\text { average accurac } \\
\text { meetings. }\end{array}$ & $\begin{array}{l}\text { found that turn- } \\
\text { lone contributing } \\
\text { improvement to } \\
\text { eativity, with an } \\
74.40 \% \text { across }\end{array}$ \\
\hline
\end{tabular}

We thus argue that this implies a communication style more reflective of negotiation, thus counterproductive to creative convergence. This is particularly interesting, considering the intersection of the two otherwise disparate areas of team collaborative interaction research along the lines of Bechtoldt, De Dreu, Nijstad and Choi (2010) who explored group information processing and epistemic social tuning.

Chapter 4 built on the fundamental work of Bernieri and Rosenthal (1991) on behavioral convergence by adding that nonverbal behavior (i.e., social signals) converges with verbal behavior (i.e., creative problem solving) acting as a new valid proxy for consensus. Methodologically speaking, we focused on introducing network alignment as a new methodology to social sciences; deep-rooted in discrete mathematics and computational biology (Koyutürk et al., 20o6; Emmert-Streib et al., 2016). 
Table 2. Overview of results for Chapter 4

Research question: How do social signals align with dyadic creativity?

\begin{tabular}{|c|c|c|c|c|c|c|}
\hline & & Class: & HCA Same & LCA Same & HCA Other & LCA Other \\
\hline \multirow{4}{*}{ Results } & TM & F-measure: & 0.87 & 0.79 & 0.83 & 0.76 \\
\hline & & Accuracy: & \multicolumn{4}{|c|}{$81.31 \%$} \\
\hline & \multirow[t]{2}{*}{ TG } & F-measure: & 0.85 & 0.83 & 0.82 & 0.82 \\
\hline & & Accuracy: & \multicolumn{4}{|c|}{$82.93 \%$} \\
\hline $\begin{array}{l}\text { Key } \\
\text { Findings }\end{array}$ & \multicolumn{6}{|c|}{$\begin{array}{l}\text { We have identified (a) network topological evidence of creative activation in teams, and, } \\
\text { (b) isolate patterns in social signals (i.e. interaction synchrony) at different points in time } \\
\text { that contribute most to this result. }\end{array}$} \\
\hline
\end{tabular}

As described in Table 6 of Chapter 4 and above in Table 2, we discovered that team members might be actively seeking to spend the most time in the immediate vicinity of those who they perceive having a creative tie with; in this case, the perceived creative tie would preexist and influence physical distance, raising important implications for managers exploring the effect of engineering heterogeneous teams for optimal creativity (Perry-Smith and Shalley, 2014). Additionally, it is possible that team members are more capable to evaluate their creative interaction with those with which they collaborated longer.

Given that the team meetings were task-focused, the time spent face-to-face could have been augmented by the assignment of expertise-specific sub-teams. The members of these subteams could conceivably have evaluated their creative ties highly due to the sub-team homophily, consistent with social network research, whereby individuals are more likely to interact with others who share some of their attributes (McPherson et al., 2001). Notably however, ascending trends of matches over time were exhibited by both teams, deriving primarily from the time spent face-to-face, the time spent in proximity, and the mirroring of voice volume. It can therefore be said the creation and usage of communication channels (i.e., turn-taking) does not exhibit significant alignment with the creative ties, but, contrarily, the way the team members communicated (i.e. the volume mirroring of their voices) sparks the creative activation.

\section{Overall Implications}

The overarching theoretical contribution to Signaling theory (Connelly et al., 2011) resides in revealing what behavioral cues most affect creative problem solving in the case of information asymmetry at micro-level. This present thesis sheds light on the effects of nonverbal behavioral cues of co-development teams. In contrast to social behaviors that manifest over longer time-spans, these cues (i.e., social signals) described how brief changes (up to a minute long) in team members' proxemics, vocalics, and kinesics affected their perceived creativity. These signals, mostly perceived and interpreted outside conscious awareness, accompany and majorly impact verbal communication. Just as how at the macrolevel one actor is concerned about another actor's behavior or behavioral intentions (Elitzur \& Gavious, 2003), at the micro-level the collaborating actors start from a state of information 
asymmetry and need to adjust the interplay of interactions to uncover latent knowledge (Connelly et al., 2011), needs, and ideas to best develop a new service. During the social interactions in the NSD processes we observed that social signals conveyed information not only for each of the team members (at dyadic level when each team member is only exposed to the signals of another team member), but they also dictated the nature of the social interaction and its outcomes (at team level when a team member is exposed to all signals from all the other team members).

These empirical studies were conducted with the aim of contributing mainly in a methodological manner to the existing literature from which it spanned, namely, (1) service research (which provided service development processes as the research context), (2) management (which provided the theoretical foundation for customer involvement as catalyst for innovation and for team dynamics and creativity), and (3) computer science (which informed the deployment of wearable technology as well as the algorithms for analysis).

As introduced in Chapter 1, we defined creativity in our context as the process of generating new and useful ideas, and specified that the output of this process is a new innovative service. We built on the growing body of literature investigating how the dynamic social interactions shape the creative process (Brennan \& Enns, 2015; De Jaegher, Di Paolo, \& Gallagher, 2010; Heerey, 2015). Acknowledging the importance of the individuals' contribution, we posit that the individual creative potential sparks in the social interaction between team members (Perry-Smith \& Shalley, 2003). We demonstrated how the role of the social interactions varies throughout the stages of creative pursuits (generation, elaboration, and implementation) with different social signals contributing differently to the creativity experienced by the members of the $\mathrm{B}_{2} \mathrm{~B}$ co-development teams. The following sections describe how these overarching implications stem from each of the constituent chapters.

\subsection{Chapter 2: Capturing the Right Signals: Method Addendum}

Chapter 2 presented the potential of social signals as new types of data for social science research in the form of audio, speech, video, language, image, multimedia, sensor, communication, geophysical, biological data collected with wearable technologies. We present the sociometric badge as a viable tool for detecting and recording a wide range of social signals (i.e., vocal social signals, posture and body movement, time spent in relative proximity and time spent face-to-face) (Olguín-Olguín, 2007). However, the drawback of this tool is, that most variables have insufficient theoretical foundations for constructing hypotheses. The solution we provide is employing machine learning algorithms "lesstraditional approach" (for social sciences) to analyzing data. To alleviate the lack of theoretical framing for hypotheses development, we discuss data-driven theory development as a viable alternative for traditional theory building approaches. The chapter concluded with a step-by-step best practice procedure of conducting a study with wearable devices and dealing with such new types of data.

\subsection{Chapter 3: It Takes Two to Tango}

This chapter took proximity and information flow as a point of departure for investigating actual face-to-face encounters between actors and objectively measured communication patterns as factors shaping creative outcomes in new service development (NSD) teams. This 
represents a contribution to literature focusing on human dynamic network structures in the context of innovation (Perry-Smith \& Shalley, 2003, 2014), by emphasizing the exploration of objective measurement of 'honest signals'. Specifically, this chapter presented preliminary evidence linking proxemics and communicative measures of 'honest social signals' as markers of creative success within teams. The multiple regressions with quadratic assignment procedure (MRQAP) was compared with multi-class classification revealing the roles of different objective measures gathered using wearable computing devices.

The results are contingent upon the interaction stage (i.e., meeting). In the first meeting, we have identified that the relative time spent in positive body movement, as a measure of behavioral convergence, is serving as a valid proxy for consensus and thus detrimental to creativity. In the second meeting, time each dyad spends facing each other in the prototyping phase influences perceived creativity only when most (if not all possible dyads) are present. In the third meeting, we show the non-desirable characteristic of creativity in this convergence stage since the communication distribution negatively influences creativity; we argue that this implies a communication style in the realm of negotiation (which is crucial to reaching consensus before the team moves on to the next project phase) rather than idea generation.

The practical implications of this chapter are two-fold; first outline the importance of nonverbal behavior in affecting as well as predicting creativity in the ideation phase of the process, proving that creativity is the outcome of complex social interactions. The main implication for service research is that the service design task (i.e., "layer") was the third most important factor contributing to an accurate prediction of perceived creativity. This implies that in the converging stage of the ideation process the structure of the project meeting itself is highly important.

\subsection{Chapter 4: For Your Ties Only}

The premise of this chapter was that some social relationships (i.e., ties) are more important for the creative process than others during the new service development process. We proceeded to investigate team interaction patterns that lead to creative problem solving by looking at how creative relationships (ties) align with objective nonverbal social behaviors over the project span. We introduce the concept of "creative activation" defined as the convergence between creative ties and social signals. The main contribution of the paper is successfully applying dynamic network alignment in social science (previously solely used in bio-informatics) to examine the real-time interactions (i.e., social signals) generated by two new service development (NSD) teams, and their participating customers during actual company meetings. This formalized comparison of networks rendered (a) network topological evidence of creative activation in teams, and, (b) isolate patterns in social signals (i.e. interaction synchrony) at different points in time that contribute most to this result.

\section{Concluding Remarks}

In line with the business literature and drawing from recent developments in computational social science, the field of 'quantified team member' will soon have the technological tools to measure focal dependent variables such as creativity. Even though self-reports represent the best to-date meeting assessment, future research will draw upon developments 
ecological momentary assessment to capture such soft features in actu and over time. The future project teams will also incorporate physiological and psychological measures that correspond with manifestations of focal dependent variables. Looking forward we can only assume the broader implications for workplace dynamics of the 'quantified team member' as well as for knowledge sharing, organizational learning, innovation, etc.

The first hindrance in achieving this new prototype of team member relies in the accuracy of measures, the data merger and analysis, as well as in the (theoretical) implication of results. This thesis is a first such attempt at using environmentally aware devices developed in the field of computer science, building upon seminal theoretical work in social psychology and management, in a field studies to explain behavioral phenomena conducive to creativity and innovation potential. We have thusly contributed to the growing body of literature focused on inspiring new approaches to creativity and innovation within teams (Elsbach \& Hargadon, 2006; Sonenshein, 2016).The distinct research fields (i.e., computer science and engineering, organizational behavior, team research, marketing, etc.) are converging towards employing tools that measure objective behaviors but, without a multidisciplinary approach to developing and testing said tools, they fall short due to the blind spots (i.e., lack of know-how) in either the theoretical foundation or the technological complexity of the collected measures (Chaffin, Heidl, Hollenbeck et al., 2017; Maglio, Kwan, \& Spohrer, 2015).

The opportunities provided by these new data collectors are only starting. Due to the highrate frequency sampling we are able to run simulations even on small teams. For instance, future research will be able to predict and causally assess the effect of removing one team member from a particular timeframe. This opens the door to researching with modern tools yet another fundamental theoretical avenue that of social permutations where individuals' activities are shaped by their sense of self, relationships with others, and social roles (Fiske, 1991).

To conclude beyond all the right signals in line with Brown (2016), creativity may not be of interest to every individual, team, or organization and it may not be only competition upholding practice. However, it definitely is the practice on which all others depend as it is indispensable to innovation. Creativity can only flourish when leadership shifts from treating new ideas as performance checks (i.e., operational competitiveness) to fostering creative competitiveness. 


\section{Chapter 6}

\section{Valorization Addendum}


I am a sociologist by training and at heart. I have been fascinated by how we, humans, interact with each other and how our interactions drive what we collectively create. During the first half of 2013 I became a deputy project leader at the Service Science Factory, where I extended my hands-on knowledge on service innovation. While at the Service Science Factory, I became fascinated about how teams work in project-based settings, characterized by multidisciplinary teams and service co-development with customers. I carried this enthusiasm over to my research and decided to further investigate how we come together to develop and deliver market-focused creative solutions to commercial challenges via new services.

The purpose of this dissertation is to shed light on how the way individuals interact and communicate with each other influence their creative endeavors and how businesses work to bring about innovation through in-group communication. When it comes to creativity, there is little theoretical consensus on its drivers. Some scholars argue it is an individual trait, others consider it a group/ team outcome. What if it is neither? A growing literature stream talks about "the social side of creativity", namely creative output as a result of a dynamic interaction process. I synthetize this process with perspectives from signaling theory in the context of organizational behavior, as well as research into so-called "honest signals". These signals are nonverbal behavioral indicators humans knowingly and unknowingly exhibit in social interactions such as tone of voice, posture, amount of body movement, face-to-face interaction time among others.

\section{The LEGO Light Bulb Metaphor}

Picture a team in a meeting room. They're tasked with solving a novel problem. Each individual has a set of ideas on how to approach and solve the issue. Picture these ideas as Lego bricks in message bubbles above their heads. Each team member's "ideas" are of different shapes and sizes, but of only one color. As they start interacting, each of them decides what idea (read "size and shape of brick") would fit best at every moment in time. If we imagine the solution to that novel problem as, say, a light bulb, the size, shape, and color pattern of this Lego light bulb depends consistently on how the team members communicate and interact with each other in the process.

\section{Social Signals}

Human social interaction mechanisms are quite ancient; signaling (nonverbal) behavior outdates language but yet still remains inextricable to communicative processes (that is why we are still talking about "dominance", "introversion", and other behavioral traits of such nature). Researchers have long studied how these signals we give away and perceive influence our interactions, but their endeavors have mostly been limited to traditional means of collecting data such as self-assessments, and potentially biased observations. In 2007, researchers at MIT Media Lab developed purpose-designed wearable sensor devices, with high measurement accuracy, making them suitable for the rigorous academic data collection and analysis in organizational behavior contexts. By means of this technology, it is now possible to collect objective, second-by-second streams of data about human behavior that help us to begin to understand patterns of human interaction in ways that weren't before 
possible. Thus, we now have appropriate means by which to capture and assess objective behaviors and improve our understanding of to what extent nonverbal communication actually influences other actors within an interactive setting, as well as how much it affects the quality of our relationships, and how much it influences our (creative) performance when we collaborate.

Through my project, I managed to bring to European academia the first 45 Sociometric badges to be utilized in social science research. I deployed these devices in real-life innovation projects involving four industrial partners in the professional services, veterinary pharmaceutics, educational, and medical technology sectors. I developed several research designs that accommodate the use of these new sensors and identified the appropriate analytical approaches across disciplines to collect and analyze this special type of data. This involves insights from domains as diverse as computer science, sociology and psychology, as well as business domains such as service research, marketing, innovation and organizational behavior.

Through my studies I carefully followed the actual nonverbal behavior of innovation teams in their creative problem solving processes, second-by-second. So, can honest signals predict creative problem solving? We found that up to $20 \%$ of teams' creativity can in fact be explained by their members' interaction patterns alone (e.g., who talks when [turn-taking], the time spent in other's proximity, the time individuals spent face-to-face, the ways they mirrored the posture and activity levels, etc.). Thanks to this newly available technology, we are now able to quantify and understand human interactions in a way not previously possible, thus contributing to development of the pervasive and ubiquitous computing via computational social science, as well as theorizing around new service development, innovation, and organizational behavior.

\section{Implications for Practice}

In line with the business literature and drawing from recent developments in computational social science, the field of 'quantified team members' will soon have the technological tools to measure focal dependent variables such as creativity. Even though self-reports represent the best to-date meeting assessment, future research will draw upon developments such as ecological momentary assessment to capture such soft features in actu and over time. Future project teams will be able to learn from physiological and psychological measures that correspond with manifestations of focal dependent variables. Looking forward we can only assume that this will have substantially broader implications for the management of workplace dynamics, knowledge sharing, organizational learning, and innovation.

The main obstacle to overcome in achieving this new perspective on team science concerns the logistical aspects of data collection, namely the management of deployments, the resources required for data collection and manipulation, and challenges concerning the establishment of ground-truths. Another challenge is to do this in a way that respects user privacy and does not risk compromising user uptake and the benefits derived from such analysis. 
To summarize, this thesis employs environmentally-aware sensor devices within a series of field studies, to capture real-time markers of team dynamics and demonstrates the value of this data to support theorizing. Here, this pertains specifically to supporting the growing body of literature around unlocking the creative and innovative potential of teams (Elsbach \& Hargadon, 2006; Sonenshein, 2016). Put simply, a number of distinct research fields (i.e., computer science and engineering, organizational behavior, team research, marketing, etc.) are converging towards employing tools that measure objective behaviors but, without a multidisciplinary approach to developing and testing said tools, they fall short due to the blind spots (i.e., lack of know-how) in either the theoretical foundation or the technological complexity of the collected measures (Chaffin, Heidl, Hollenbeck et al., 2017; Maglio, Kwan, \& Spohrer, 2015).

The opportunities provided by these new data collection tools are in relative terms, only just beginning to become apparent. Given the potential for high-rate frequency sampling we are able to run quasi-simulations even on small teams to further supplement primary empirical investigation. For instance, future research will be able to predict and causally assess the effect of removing one team member from a particular timeframe. This opens the door to researching with modern tools yet another fundamental theoretical avenue that of social permutations where individuals' activities are shaped by their sense of self, relationships with others, and social roles (Fiske, 1991).

To conclude, beyond all the right signals, and in line with Brown (2016), creativity may not be of interest to every individual, team, or organization, and it may not always be deemed the most critical competitive focal point. However, creativity definitely is the practice on which all other practices ultimately depend as it is indispensable to innovation; the lifeblood of commercial continuity. It should be noted though that creativity can only flourish when leadership shifts from treating new ideas as performance checks (i.e., operational competitiveness) to fostering true creative competitiveness, managed via a detailed understanding of the interaction between engaged actors and beneficiaries. 


\section{Bibliography}


Adami, C. (2004). The physics of information. arXiv preprint quant-ph/0405005.

Adami, C., \& Hintze, A. (2013). Evolutionary instability of zero-determinant strategies demonstrates that winning is not everything. Nature Communications, 4, 2193.

Afuah, A. (2003). Innovation management: strategies, implementation and profits. Oxford University Press.

Ahuja, G. (200o). Collaboration networks, structural holes, and innovation: A longitudinal study. Administrative Science Quarterly, 45(3), 425-455.

Akerlof, G. A. (1970). The Market for Lemons: Quality Uncertainty and the Market Mechanism. Quarterly Journal of Economics, 84(488).

Alavi, M., \& Leidner, D. E. (2001). Knowledge management and knowledge management systems: Conceptual foundations and research issues. MIS quarterly, 107-136.

Albert, R., \& Barabási, A. L. (2002). Statistical mechanics of complex networks. Reviews of Modern Physics, 74(1), 47.

Alexander, L., \& Van Knippenberg, D. (2014). Teams in pursuit of radical innovation: A goal orientation perspective. Academy of Management Review, 39 (4), 423-438.

Allen, T. J. (1977). Managing the flow of technology. Cambridge, MA: The MIT Press.

Allport, G. W. (1937). Personality: A psychological interpretation. Holt

Almetric. (2019). Retrieved from https://www.altmetric.com/top10o/2018/

Amabile, T. M. (1983). The social psychology of creativity: A componential conceptualization. Journal of Personality and Social Psychology, 45(2), 357.

(1996). Creativity in context: Update to the social psychology of creativity. Hachette UK.

\& Pratt, M. G. (2016). The dynamic componential model of creativity and innovation in organizations: Making progress, making meaning. Research in Organizational Behavior, 36, 157-183.

Ambady, N., Bernieri, F. J., \& Richeson, J. A. (2000). Toward a histology of social behavior: Judgmental accuracy from thin slices of the behavioral stream. Advances in Experimental Social Psychology, 32, 201-271.

Anderson, N., De Dreu, C. K., \& Nijstad, B. A. (2004). The routinization of innovation research: A constructively critical review of the state-of-the-science. Journal of Organizational Behavior, 25(2), 147-173.

\section{$\boldsymbol{B}$}

Baer, M., Evans, K., Oldham, G. R., \& Boasso, A. (2015). The social network side of individual innovation: A meta-analysis and path-analytic integration. Organizational Psychology Review, doi: 2041386614564105.

Baldwin, C., \& von Hippel, E. (2011). Modeling a paradigm shift: From producer to user innovation and open collaborative innovation. Organization Science, 22 (6), 13991417.

Banse, R., \& Scherer, K. R. (1996). Acoustic profiles in vocal emotion expression. Journal of Personality and Social Psychology, 7o(3), 614. 
Barry, B., \& Crant, JM (200o). Dyadic communication relationships in organizations: An attribution / expectancy approach. Organization Science, 11 (6), 648-664.

Basseville, M. (1989). Distance measures for signal processing and pattern recognition. Signal Processing, 18(4), 349-369.

Basu, A. (2002). Conversational Scene Analysis. Massachusetts Institute of Technology, Boston, MA.

Baur, T., Damian, I., Lingenfelser, F., Wagner, J., \& André, E. (2013, October). Nova: Automated analysis of nonverbal signals in social interactions. In International Workshop on Human Behavior Understanding (pp. 160-171). Springer International Publishing.

Bechtoldt, M.N., De Dreu, C.K., Nijstad, B.A. and Choi, H.S., 2010. Motivated information processing, social tuning, and group creativity. Journal of Personality and Social Psychology, 99(4), 622.

Bermingham, M. L., Pong-Wong, R., Spiliopoulou, A., Hayward, C., Rudan, I., Campbell, H., ... \& Haley, C. S. (2015). Application of high-dimensional feature selection: evaluation for genomic prediction in man. Scientific Reports, 5, 10312.

Bernieri, F. J., \& Rosenthal, R. (1991). 11. Interpersonal coordination: Behavior matching and interactional synchrony. Fundamentals of Nonverbal Behavior, 401.

Bernstein, E. S., \& Turban, S. (2018). The impact of the 'open' workspace on human collaboration. Philosophical Transactions of the Royal Society B: Biological Sciences, 373(1753), 20170239.

Bersin, J., Mariani, J., Monahan, K. (2016) Will IoT technology bring us the quantified employee? The Internet of Things in human resources. Deloitte University Press, Retrieved from https://dupress.deloitte.com/dup-us-en/focus/internet-ofthings/people-analytics-iot-human-resources.html\#endnote-11

Bharadwaj, S., \& Menon, A. (200o). Making innovation happen in organizations: individual creativity mechanisms, organizational creativity mechanisms or both? Journal of Product Innovation Management, 17(6), 424-434.

Bickel, P. J., Ritov, Y. A., \& Stoker, T. M. (2006). Tailor-made tests for goodness of fit to semiparametric hypotheses. The Annals of Statistics, 34(2), 721-741.

Biemans, W. G., Griffin, A., \& Moenaert, R. K. (2016). Perspective: New service development: How the field developed, its current status and recommendations for moving the field forward. Journal of Product Innovation Management, 33(4), 382-397.

Bird, R., Smith, E., Alvard, M., Chibnik, M., Cronk, L., Giordani, L., ... \& Smith, E. (2005). Signaling theory, strategic interaction, and symbolic capital. Current Anthropology, 46(2), 221-248.

Birdwhistell, R. L. (1952). Introduction to kinesics: An annotation system for analysis of body motion and gesture. Department of State, Foreign Service Institute.

Blei, D. M., \& Smyth, P. (2017). Science and data science. Proceedings of the National Academy of Sciences, 114(33), 8689-8692.

Bogers, M., Afuah, A., \& Bastian, B. (2010). Users as innovators: A review, critique, and future research directions. Journal of Management, 36(4), 857-875.

Borgatti, S.P., Everett, M.G. \& Freeman, L.C. (2002). UciNET for Windows: Software for Social Network Analysis. Harvard, MA: Analytic Technologies. 
Bousmalis, K., Mehu, M., \& Pantic, M. (2009, September). Spotting agreement and disagreement: A survey of nonverbal audiovisual cues and tools. In Affective Computing and Intelligent Interaction and Workshops, 2009. ACII 2009. 3rd International Conference on (pp. 1-9). IEEE.

Breiman, L. (2001a). Random forests. Machine Learning, 45(1), 5-32.

(2001b). Statistical modeling: The two cultures (with comments and a rejoinder by the author). Statistical Science, 16(3), 199-231.

Brennan, A. A., \& Enns, J. T. (2015). What's in a friendship? Partner visibility supports cognitive collaboration between friends. PloS One, 10(11), e0143469.

Brockhoff, K. (2003). Customers' perspectives of involvement in new product development. International Journal of Technology Management, 26(5-6), 464-481.

Brown, C., Efstratiou, C., Leontiadis, I., Quercia, D., Mascolo, C., Scott, J., \& Key, P. (2014, September). The architecture of innovation: Tracking face-to-face interactions with ubicomp technologies. In Proceedings of the 2014 ACM International Joint Conference on Pervasive and Ubiquitous Computing (pp. 811-822). ACM.

Brown, J. S., \& Duguid, P. (1991). Organizational learning and communities-of-practice: Toward a unified view of working, learning, and innovation. Organization Science, 2(1), 40-57.

Brown, T. (2016). Leaders Can Turn Creativity into a Competitive Advantage. Harvard Business Review. Retrieved from https://hbr.org/2016/11/leaders-can-turn-creativityinto-a-competitive-advantage Bryant, A., \& Charmaz, K. (Eds.). (2007). The Sage handbook of grounded theory. Sage.

Bstieler, L., \& Hemmert, M. (2010). Increasing Learning and Time Efficiency in Interorganizational New Product Development Teams. Journal of Product Innovation Management, 27(4), 485-499.

Buchanan, M. (2007). The Science of Subtle Signals. Strategy and Business, 48, 68.

Burgoon, J. K., Guerrero, L. K., \& Floyd, K. (2016). Nonverbal Communication. Routledge.

Bzdok, D., Altman, N., \& Krzywinski, M. (2018). Points of significance: statistics versus machine learning. Nature Methods, 1-7.

C

Callegaro, M., \& Yang, Y. (2018). The Role of Surveys in the Era of "Big Data”. In The Palgrave Handbook of Survey Research (pp. 175-192). Palgrave Macmillan, Cham.

Carbonell, P., Rodríguez-Escudero, A. I., \& Pujari, D. (2009). Customer involvement in new service development: An examination of antecedents and outcomes. Journal of Product Innovation Management, 26(5), 536-550.

Carmeli, A. Gelbard, R., \& Reiter-Palmon, R. (2013). Leadership, creative problem-solving capacity, and creative performance: The importance of knowledge sharing. Human Resource Management, 52 (1), 95-121.

Chaffin, D., Heidl, R., Hollenbeck, J. R., Howe, M., Yu, A., Voorhees, C., \& Calantone, R. (2017). The promise and perils of wearable sensors in organizational research. Organizational Research Methods, 2o(1), 3-31.

Chang, W., \& Taylor, S. A. (2016). The effectiveness of customer participation in new product development: A meta-analysis. Journal of Marketing, 8o(1), 47-64. 
Chartrand, T. L., \& Bargh, J. A. (1999). The chameleon effect: the perception-behavior link and social interaction. Journal of Personality and Social Psychology, 76(6), 893.

Chawla, N. V., Bowyer, K. W., Hall, L. O., \& Kegelmeyer, W. P. (2002). SMOTE: synthetic minority over-sampling technique. Journal of Artificial Intelligence Research, 16, 321357.

Chesbrough, H. W. (2006). Open innovation: The new imperative for creating and profiting from technology. Harvard Business Press.

Choudhary, T. (2004). Sensing and Modeling Human Networks. Massachusetts Institute of Technology, Cambridge, MA.

\& Pentland, A. (2004). Characterizing social networks using the sociometer. Proceedings of the North American Association of Computational Social and Organizational Science (NAACSOS).

Christensen, C. (2013). The innovator's dilemma: when new technologies cause great firms to fail. Harvard Business Review Press.

Christensen, C. M., \& Bower, J. L. (1996). Customer power, strategic investment, and the failure of leading firms. Strategic Management Journal, 17(3), 197-218.

Chronaki, G., Wigelsworth, M., Pell, M. D., \& Kotz, S. A. (2018). The development of crosscultural recognition of vocal emotion during childhood and adolescence. Scientific reports, $8(1), 8659$.

Comte, A. (1856). Social physics: from the Positive philosophy of Auguste Comte. C. Blanchard.

Connelly, B. L., Certo, S. T., Ireland, R. D., \& Reutzel, C. R. (2011). Signaling theory: A review and assessment. Journal of Management, 37(1), 39-67.

Consultancy.uk. (2018). Retrieved from https://www.consultancy.uk/news/17924/consultants-and-agencies-in-an-armsrace-as-industry-boundaries-blur

Conway, H. A., \& McGuinness, N. W. (1986). Idea generation in technology-based firms. Journal of Product Innovation Management, 3(4), 276-291.

Coombs, R., \& Miles, I. (200o). Innovation, measurement and services: the new problematique. In Innovation systems in the service economy (pp. 85-103). Springer, Boston, MA.

Cristani, M., Raghavendra, R., Del Bue, A., \& Murino, V. (2013). Human behavior analysis in video surveillance: A social signal processing perspective. Neurocomputing, 10o, 8697 .

Cromwell, J. R., Amabile, T. M., \& Harvey, J. F. (2018). An Integrated Model of Dynamic Problem Solving Within Organizational Constraints. In Individual Creativity in the Workplace (pp. 53-81). Academic Press.

Cui, A. S., \& Wu, F. (2015). Utilizing customer knowledge in innovation: antecedents and impact of customer involvement on new product performance. Journal of the Academy of Marketing Science, 1-23.

(2017). The impact of customer involvement on new product development: Contingent and substitutive effects. Journal of Product Innovation Management, 34(1), 6o-80. 


\section{D}

Darwin, C., \& Prodger, P. (1998). The expression of the emotions in man and animals. Oxford University Press, USA.

Davis, M. A. (2009). Understanding the relationship between mood and creativity: A metaanalysis. Organizational Behavior and Human Decision Processes, 108(1), 25-38.

de Borst, A. W., \& de Gelder, B. (2016). Clear signals or mixed messages: inter-individual emotion congruency modulates brain activity underlying affective body perception. Social Cognitive and Affective Neuroscience, 11(8), 1299-1309.

De Clercq, D., Mohammad Rahman, Z., \& Belausteguigoitia, I. (2017). Task conflict and employee creativity: The critical roles of learning orientation and goal congruence. Human Resource Management, 56(1), 93-109.

De Jaegher, H., Di Paolo, E., \& Gallagher, S. (2010). Can social interaction constitute social cognition? Trends in Cognitive Sciences, 14(10), 441-447.

de Montjoye, Y. A., Stopczynski, A., Shmueli, E., Pentland, A., \& Lehmann, S. (2014). The strength of the strongest ties in collaborative problem solving. Scientific Reports, 4, 5277.

Dekker, D., Krackhardt, D., \& Snijders, T. A. (2007). Sensitivity of MRQAP tests to collinearity and autocorrelation conditions. Psychometrika, 72(4), 563-581.

Deutsch, F. (1947). Analysis of postural behavior. The Psychoanalytic Quarterly, 16(2), 195213.

(1952). Analytic posturology. The Psychoanalytic Quarterly, 21(2), 196-214.

Dietzel, J., Francu, R. E., Lucas, B., \& Zaki, M. (2018). Contextually Defined Postural Markers Reveal Who's in Charge: Evidence from Small Teams Collected with Wearable Sensors. Cambridge Service Alliance Working Paper, January 2018. Retrieved from: https://cambridgeservicealliance.eng.cam.ac.uk

Djeddi, W. E., Yahia, S. B., \& Nguifo, E. M. (2018). A Novel Computational Approach for Global Alignment for Multiple Biological Networks. IEEE/ACM Transactions on Computational Biology and Bioinformatics.

Drejer, I. (2004). Identifying innovation in surveys of services: A Schumpeterian perspective. Research Policy, 33(3), 551-562.

Dul, J., Ceylan, C., \& Jaspers, F. (2011). Knowledge workers' creativity and the role of the physical work environment. Human Resource Management, 5o(6), 715-734.

E

Earley, P. C., \& Mosakowski, E. (2004). Cultural intelligence. Harvard Business Review, 82(10), 139-146.

Eberly, MB, Holley, EC, Johnson, MD, \& Mitchell, TR (2011). Beyond internal and external: A dyadic theory of relational attributions. Academy of Management Review, 36 (4), 731753.

Eilers, S. (2016). The LEGO Counting Problem. American Mathematical Monthly, 123(5), 415426. 
Ekman P. (2004) Emotional and Conversational Nonverbal Signals. In: Larrazabal J.M., Miranda L.A.P. (eds) Language, Knowledge, and Representation. Philosophical Studies Series, vol 99. Springer, Dordrecht

Elitzur, R., \& Gavious, A. (2003). Contracting, signaling, and moral hazard: a model of entrepreneurs, 'angels', and venture capitalists. Journal of Business Venturing, 18(6), 709-725.

Elsbach, KD, \& Hargadon, AB (2006). Enhancing creativity through "mindless" work: A framework design or workday. Organization Science, 17 (4), 470-483.

Emmert-Streib, F., Dehmer, M., \& Shi, Y. (2016). Fifty years of graph matching, network alignment and network comparison. Information Sciences, 346, 180-197.

Enfield, N. J., \& Levinson, S. C. (2006). Roots of human sociality. New York: Berg.

$F$

Fang, E. (2008). Customer participation and the trade-off between new product innovativeness and speed to market. Journal of Marketing, 72(4), 90-104

Feldman, R. S., Feldman, R. S., \& Rimé, B. (Eds.). (1991). Fundamentals of nonverbal behavior. Cambridge University Press.

Ferrin, D. L., Dirks, K. T., \& Shah, P. P. (2006). Direct and indirect effects of third-party relationships on interpersonal trust. Journal of Applied Psychology, 91(4), 870.

Fiske, A. P. (1991). Structures of social life: The four elementary forms of human relations: Communal sharing, authority ranking, equality matching, market pricing. New York, NY, US: Free Press.

Flach, P. (2012). Machine learning: the art and science of algorithms that make sense of data. Cambridge University Press.

Flikkema, M., Jansen, P., \& Van Der Sluis, L. (2007). Identifying neo-Schumpeterian innovation in service firms: A conceptual essay with a novel classification. Economics of Innovation and New Technology, 16(7), 541-558.

Fong, C. T. (2006). The effects of emotional ambivalence on creativity. Academy of Management Journal, 49(5), 1016-1030.

Frank, M. G., Griffin, D. J., Svetieva, E., \& Maroulis, A. (2014). Nonverbal Elements of the Voice. The Social Psychology of Nonverbal Communication, 92.

Franke, N., Keinz, P., \& Steger, C. J. (2009). Testing the value of customization: when do customers really prefer products tailored to their preferences? Journal of Marketing, 73(5), 103-121.

Fredrickson, B. L. (2001). The role of positive emotions in positive psychology: The broadenand-build theory of positive emotions. American Psychologist, 56(3), 218.

Fromm-Reichmann, F. (1950). Principles of intensive psychotherapy. Chicago (University of Chicago Press) 1950.

Fu, N., Flood, P. C., Bosak, J., Rousseau, D. M., Morris, T., \& O'Regan, P. (2017). HighPerformance work systems in professional service firms: Examining the practicesresources-uses-performance linkage. Human Resource Management, 56(2), 329-352. 


\section{G}

Gallouj, F. (2002). Services innovation: assimilation, differentiation, inversion and integration. In (Published in Bidgoli H. (Ed.), (2010). The Handbook of Technology Management. John Wiley and Sons, p. 989-1000

Gargiulo, M., \& Benassi, M. (200o). Trapped in your own net? Network cohesion, structural holes, and the adaptation of social capital. Organization Science, 11(2), 183-196.

George, G., Osinga, EC, Lavie D., and Scott, BA (2016). Big data and data science methods for research management. Academy of Management Journal, 59 (5), 1493-1507.

George, J. M., \& Zhou, J. (2002). Understanding when bad moods foster creativity and good ones don't: the role of context and clarity of feelings. Journal of Applied Psychology, 87(4), 687 .

Gibbons, D. E. (2004). Network structure and innovation ambiguity effects on diffusion in dynamic organizational fields. Academy of Management Journal, 47(6), 938-951.

Giles, H. (1979). Ethnicity markers in social class. In Scherer, K. R. and Giles, H. (Eds.). Social markers in speech. Cambridge University Press. 251-289

Gilson, LL, \& Shalley, CE (2004). A little creativity goes a long way: An examination of teams' involvement in creative processes. Journal of Management, 30 (4), 453-470.

Gloor, P. A., Almozlino, A., Inbar, O., Lo, W., \& Provost, S. (2014). Measuring Team Creativity Through Longitudinal Social Signals. arXiv preprint arXiv:1407.0440.

Gonzales, A. L., Hancock, J. T., \& Pennebaker, J. W. (2010). Language style matching as a predictor of social dynamics in small groups. Communication Research, 37(1), 3-19.

Grabner, I. (2014). Incentive system design in creativity-dependent firms. The Accounting Review, 89(5), 1729-1750.

\& Speckbacher, G. (2016). The cost of creativity: A control perspective. Accounting, Organizations and Society, 48, 31-42.

\section{H}

Haig, B. D. (1995). Grounded theory as scientific method. Philosophy of Education, 28(1), 1-11.

Hall, E. T. (1966). The Hidden Dimension. Anchor Books.

(1963). A System for the Notation of Proxemic Behavior. American Anthropologist. 65, 5, 1003-1026.

Hanneman RA, Riddle M (2005) Introduction to social network methods. Riverside, CA: University of California, Riverside.

Harrison, F., Sciberras, J., \& James, R. (2011). Strength of social tie predicts cooperative investment in a human social network. PLoS One, 6(3), e18338.

Harvey, S. (2014). Creative synthesis: Exploring the process of extraordinary group creativity. Academy of Management Review, 39(3), 324-343.

\& Kou, C. Y. (2013). Collective engagement in creative tasks the role of evaluation in the creative process in groups. Administrative Science Quarterly, 58(3), 346-386. 
He, L. (2013). Google's Secrets of Innovation: Empowering Its Employees. Retrieved from http://www.forbes.com/sites/laurahe/2013/03/29/googles-secrets-of-innovationempowering-its-employees/\#fcff7f37eb39

Heerey, E. A. (2015). Decoding the dyad: Challenges in the study of individual differences in social behavior. Current Directions in Psychological Science, 24(4), 285-291.

Helfrich, H. (1979). Age markers in speech. In Scherer, K. R. and Giles, H. (Eds.). Social markers in speech. Cambridge University Press. 63-107

Hennessey, B.A., Amabile, T.M. (2010). Creativity. Annual Review of Psychology, 61,569-598

Heyden, M. L., Reimer, M., \& Van Doorn, S. (2017). Innovating beyond the horizon: CEO career horizon, top management composition, and R\&D intensity. Human Resource Management, 56(2), 205-224.

Hipp, C., \& Grupp, H. (2005). Innovation in the service sector: The demand for servicespecific innovation measurement concepts and typologies. Research policy, 34(4), 517-535.

Hoegl, M., \& Parboteeah, P. (2006). Autonomy and teamwork in innovative projects. Human Resource Management, 45 (1), 67-79.

Hülsheger, U. R., Anderson, N., \& Salgado, J. F. (2009). Team-level predictors of innovation at work: a comprehensive meta-analysis spanning three decades of research. Journal of Applied Psychology, 94(5), 1128.

$I$

IBM. (2016). Global C-suite Study - The CMO Point of View. Retrieved from http://www935.ibm.com/ services/c-suite/study/studies/cmo-study/

Isen, A. M., Daubman, K. A., \& Nowicki, G. P. (1987). Positive affect facilitates creative problem solving. Journal of Personality and Social Psychology, 52(6), 1122.

Iyer, B., \& Davenport, T. H. (2008). Reverse Engineering: Google's Innovation Machine. Harvard Business Review, 86(4).

$\boldsymbol{J}$

Jack, S. L. (2005). The role, use and activation of strong and weak network ties: A qualitative analysis. Journal of Management Studies, 42(6), 1233-1259.

James, W. T. 1932. A study of the expression of bodily posture. Journal of General Psychology, 7405-437.

Jordan, M. I., \& Mitchell, T. M. (2015). Machine learning: Trends, perspectives, and prospects. Science, 349(6245), 255-260.

\section{K}

Kandasamy, N., Hardy, B., Page, L., Schaffner, M., Graggaber, J., Powlson, A. S., ... \& Coates, J. (2014). Cortisol shifts financial risk preferences. Proceedings of the National Academy of Sciences, 111(9), 3608-3613.

Kao, H. L. C., \& Schmandt, C. (2015, January). MugShots: A mug display for front and back stage social interaction in the workplace. In Proceedings of the Ninth International Conference on Tangible, Embedded, and Embodied Interaction (pp. 57-60). ACM. 
Kappas, A., Hess, U., \& Scherer, K. R. (1991). Voice and emotion. In Feldman, R. S., Feldman, R. S., \& Rimé, B. (Eds.). Fundamentals of nonverbal behavior. Cambridge University Press. 200

Kapucu, N. (2009). Interorganizational coordination in complex environments of disasters: The evolution of intergovernmental disaster response systems. Journal of Homeland Security and Emergency Management, 6(1).

\& $\mathrm{Hu}, \mathrm{Q}$. (2016). Understanding multiplexity of collaborative emergency management networks. The American Review of Public Administration, 46(4), 399417.

Karpatne, A., Atluri, G., Faghmous, J. H., Steinbach, M., Banerjee, A., Ganguly, A., ... \& Kumar, V. (2017). Theory-guided data science: A new paradigm for scientific discovery from data. IEEE Transactions on Knowledge and Data Engineering, 29(10), 2318-2331.

Kelle, U. (2010). The development of categories: Different approaches in grounded theory. The Sage handbook of grounded theory. Sage. 2, 191-213.

Kendon, A. (1980). Gesticulation and speech: Two aspects of the process of utterance. The Relationship of Verbal and Nonverbal Communication, 25(1980), 207-227.

Kendon, A., \& Sigman, S. J. (1996). Commemorative essay. Ray L. Birdwhistell (19181994). Semiotica, 112(3-4), 231-262.

Kim, T., McFee, E., Olguin, D. O., Waber, B., \& Pentland, A. (2012). Sociometric badges: Using sensor technology to capture new forms of collaboration. Journal of Organizational Behavior, 33(3), 412-427.

King, N., Anderson, N., (1990). Innovation in Working groups. In: West, M.A., Farr, J.L. (Eds.), Innovation and Creativity at Work. Wiley, Chichester, 81-10o.

Koyutürk, M., Kim, Y., Topkara, U., Subramaniam, S., Szpankowski, W., \& Grama, A. (2006). Pairwise alignment of protein interaction networks. Journal of Computational Biology, 13(2), 182-199.

Kozlowski, S. W. (2015). Advancing research on team process dynamics Theoretical, methodological, and measurement considerations. Organizational Psychology Review, 5(4), 270-299.

Krackhardt, D. (1987). Cognitive social structures. Social networks, 9(2), 109-134.

Krackhardt, D. (1992). A caveat on the use of the quadratic assignment procedure. Journal of quantitative anthropology, 3(4), 279-296.

Kuchaiev, O., Stevanović, A., Hayes, W., \& Pržulj, N. (2011). GraphCrunch 2: Software tool for network modeling, alignment and clustering. BMC bioinformatics, 12(1), 24.

\section{$L$}

Lee, J., Rowlands, D., Jackson, N., Leadbetter, R., Wada, T., \& James, D. A. (2017). An Architectural Based Framework for the Distributed Collection, Analysis and Query from Inhomogeneous Time Series Data Sets and Wearables for Biofeedback Applications. Algorithms, 10(1), 23.

Lepri, B., Oliver, N., Letouzé, E., Pentland, A., \& Vinck, P. (2017). Fair, Transparent, and Accountable Algorithmic Decision-making Processes. Philosophy \& Technology, 1-17.

Levinson, S. C. (2006). On the human" interaction engine". In Wenner-Gren Foundation for Anthropological Research, Symposium 134 (pp. 39-69). Berg. 
(2016). Turn-taking in human communication-origins and implications for language processing. Trends in Cognitive Sciences, 20(1), 6-14.

Li, F., Deng, H., Leung, K., \& Zhao, Y. (2017). Is perceived creativity-reward contingency good for creativity? The role of challenge and threat appraisals. Human Resource Management, 56(4), 693-709.

Lin, C. P., He, H., Baruch, Y., \& Ashforth, B. E. (2016). The effect of team affective tone on team performance: the roles of team identification and team cooperation. Human Resource Management.

Linder, J. C., Jarvenpaa, S. L., \& Davenport, T. H. (2003). Toward an innovation sourcing strategy. MIT Sloan Management Review, 44(4), 43-49.

Lipton, Z. C. (2016). The mythos of model interpretability. Proceedings of ICML Workshop on Human Interpretability in Machine Learning. Retrieved from arXiv preprint arXiv:1606.03490.

Liu, C. M., \& Donath, J. S. (2006, April). Urbanhermes: social signaling with electronic fashion. In Proceedings of the SIGCHI conference on Human Factors in computing systems (pp. 885-888). ACM.

Liu, C. Y., \& Zheng, Z. Y. (1989). Stabilization coefficient of random variable. Biometrical Journal, 31(4), 431-441.

Liu, Y., Keller, R. T., \& Shih, H. A. (2011). The impact of team-member exchange, differentiation, team commitment, and knowledge sharing on R\&D project team performance. RED Management, 41(3), 274-287.

Loth, S., \& De Ruiter, J. P. (2016). Editorial: Understanding Social Signals: How Do We Recognize the Intentions of Others? Frontiers in Psychology, 7.

Lowen, A. (1958). Physical dynamics of character structure (p. 238). New York: Grune \& Stratton.

Luria, G., \& Kalish, Y. (2013). A social network approach to peer assessment: Improving predictive validity. Human Resource Management, 52(4), 537-560.

\section{$M$}

Maglio, P. P., Kwan, S. K., \& Spohrer, J. (2015). Commentary-Toward a research agenda for human-centered service system innovation. Service Science, 7(1), 1-10.

Mahr, D., Lievens, A., \& Blazevic, V. (2014). The value of customer cocreated knowledge during the innovation process. Journal of Product Innovation Management, 31(3), 599615 .

Manjunath, B. S., Ohm, J. R., Vasudevan, V. V., \& Yamada, A. (2001). Color and texture descriptors. IEEE Transactions on circuits and systems for video technology, 11(6), 703715.

Martinez, L., Falvello, V. B., Aviezer, H., \& Todorov, A. (2016). Contributions of facial expressions and body language to the rapid perception of dynamic emotions. Cognition and Emotion, 30(5), 939-952.

Mashhadi, A., Kawsar, F., Mathur, A., Dugan, C., \& Shami, N. S. (2016, February). Let's Talk About the Quantified Workplace. In Proceedings of the 19th ACM Conference on Computer Supported Cooperative Work and Social Computing Companion (pp. 522528). ACM. 
Mateus, S. (2017). Metacommunication as Second Order Communication. KOME: An International Journal of Pure Communication Inquiry, 5(1), 80-90.

Mathieu, J. E., Hollenbeck, J. R., van Knippenberg, D., \& Ilgen, D. R. (2017). A century of work teams in the Journal of Applied Psychology. Journal of Applied Psychology, $102(3), 452$.

Tannenbaum, S. I., Donsbach, J. S., \& Alliger, G. M. (2014). A review and integration of team composition models: Moving toward a dynamic and temporal framework. Journal of Management, 40(1), 130-160.

McCrae, R. R. (1987). Creativity, divergent thinking, and openness to experience. Journal of Personality and Social Psychology, 52(6), 1258.

McDowell, T., Agarwal, D., Miller, D., Okamoto, T., Page, T. (February, 2016). Organizational design: The rise of teams. Deloitte Retrieved from https://dupress.deloitte.com/dupus-en/focus/human-capital-trends/2016/organizational-models-network-ofteams.html

McPherson, M., Smith-Lovin, L., \& Cook, J. M. (2001). Birds of a feather: Homophily in social networks. Annual Review of Sociology, 27(1), 415-444.

Mehrabian, A., \& Friar, J. T. (1969). Encoding of attitude by a seated communicator via posture and position cues. Journal of Consulting and Clinical Psychology, 33(3), 330.

Menor, L. J., Tatikonda, M. V., \& Sampson, S. E. (2002). New service development: areas for exploitation and exploration. Journal of Operations Management, 20(2), 135-157.

Minor, D., Brook, P., \& Bernoff, J. (2017). Data From 3.5 Million Employees Shows How Innovation Really Works. Harvard Business Review, Retrieved from https://hbr.org/2017/10/data-from-3-5-million-employees-shows-how-innovationreally-works on January 2018.

Mittelstadt, B., Russell, C., \& Wachter, S. (2018). Explaining Explanations in AI. Proceedings of the ACM Conference on Fairness, Accountability, and Transparency (ACM FAT*) Retrieved from https://arxiv.org/pdf/1811.01439.pdf

Montanari, A., Mashhadi, A., Mathur, A., Kawsar, F., Nawaz, S., Mascolo, C., \& Sailer, K. (2016, February). Alessandro Montanari. In International Workshop on Quantified Workplace. Co-located with CSCW.

Nawaz, S., Mascolo, C., \& Sailer, K. (2017). A Study of Bluetooth Low Energy Performance for Human Proximity Detection in the Workplace. IEEE Pervasive Computing.

Tian, Z., Francu, E., Lucas, B., Jones, B., Zhou, X., \& Mascolo, C. (2018). Measuring interaction proxemics with wearable light tags. Proceedings of the ACM on Interactive, Mobile, Wearable and Ubiquitous Technologies, 2(1), 25.

Montavon, G., Samek, W., \& Müller, K. R. (2017). Methods for interpreting and understanding deep neural networks. Digital Signal Processing. 73. 1-15

Morrison, E. W. (2002). Newcomers' relationships: The role of social network ties during socialization. Academy of Management Journal, 45(6), 1149-1160.

Moura, J. (2009). What is signal processing? [President's Message]. IEEE Signal Processing Magazine, 26(6), 6-6.

Munoz, M. A., Villanova, L., Baatar, D., \& Smith-Miles, K. (2018). Instance spaces for machine learning classification. Machine Learning, 107(1), 109-147. 
Nambisan, S. (2002). Designing virtual customer environments for new product development: Toward a theory. Academy of Management Review, 27(3), 392-413.

\section{$\boldsymbol{O}$}

Ohala, J. J. (1981). The nonlinguistic component of speech. In Darby, J. K. (Ed.). Speech evaluation in psychiatry. Grune \& Stratton, Incorporated, 39-49.

Olguín-Olguín, D. (2007). Sociometric badges: wearable technology for measuring human behavior (Doctoral dissertation, Massachusetts Institute of Technology).

Waber, B. N., Kim, T., Mohan, A., Ara, K., \& Pentland, A. (2009). Sensible organizations: Technology and methodology for automatically measuring organizational behavior. IEEE Transactions on Systems, Man, and Cybernetics, Part B (Cybernetics), 39(1), 43-55.

Oller, D. K. (1973). The effect of position in utterance on speech segment duration in English. The journal of the Acoustical Society of America, 54(5), 1235-1247.

Olsen, KM, Sverdrup, T., Nesheim, T., \& Kallenberg, AL (2016). Multiple foci of commitment in a professional service firm: balancing complex employment relations. Human Resource Management Journal, 26 (4), 390-407.

Ommen, N. O., Blut, M., Backhaus, C., \& Woisetschläger, D. M. (2016). Toward a better understanding of stakeholder participation in the service innovation process: More than one path to success. Journal of Business Research, 69(7), 2409-2416.

Onnela, J. P., Waber, B. N., Pentland, A., Schnorf, S., \& Lazer, D. (2014). ERRATUM: Using sociometers to quantify social interaction patterns. Scientific Reports, 4.

Osmond, H., (1957). Function as the basis of psychiatric ward design. Psychiatric Services, 8(4), 23-27.

\section{$\boldsymbol{P}$}

Painsky, A., \& Rosset, S. (2017). Cross-Validated Variable Selection in Tree-Based Methods Improves Predictive Performance. IEEE transactions on pattern analysis and machine intelligence, 39(11), 2142-2153.

Pease, A., \& Pease, B. (2016). The Definitive Book of Body Language: how to read others' attitudes by their gestures. Hachette UK.

Pentland, A. (2008). Honest Signals. Cambridge, Massachusetts: The MIT Press.

(2009). Reality Mining of Mobile Communications: Toward a New Deal on Data. The Global Information Technology Report (75-80).

(2012). The new science of building great teams. Harvard Business Review, 9o(4), 60-69.

(2015). Social Physics: How social networks can make us smarter. Penguin.

Perry-Smith, J. E., \& Shalley, C. E. (2003). The social side of creativity: A static and dynamic social network perspective. Academy of Management Review, 28(1), 89-106. 
\& Shalley, C. E. (2014). A social composition view of team creativity: The role of member nationality-heterogeneous ties outside of the team. Organization Science, 25(5), 1434-1452.

\& Mannucci, PV (2017). From creativity to innovation: The social network drivers of the four phases of the idea journey. Academy of Management Review, 42 (1), 53-79.

Pickles, J. (Ed.). (1995). Ground truth: The social implications of geographic information systems. Guilford Press.

Pinto, M. B., Pinto, J. K., \& Prescott, J. E. (1993). Antecedents and consequences of project team cross-functional cooperation. Management Science, 39(10), 1281-1297.

Poetz, M. K., \& Schreier, M. (2012). The value of crowdsourcing: can users really compete with professionals in generating new product ideas?. Journal of Product Innovation Management, 29(2), 245-256.

Poggi, I., \& D'Errico, F. (2010). Dominance in political debates. Human behavior understanding, LNCS, 6219.

\& Francesca, D. E. (2010, October). Cognitive modelling of human social signals. In Proceedings of the 2nd International Workshop On Social Signal Processing (pp. 21-26). ACM.

Ponsot, E., Burred, J. J., Belin, P., \& Aucouturier, J. J. (2018). Cracking the social code of speech prosody using reverse correlation. Proceedings of the National Academy of Sciences, 115(15), 3972-3977.

Portes, A. (1998). Social capital: Its origins and applications in modern sociology. Annual Review of Sociology, 24(1), 1-24.

Powers, D M W (2011). "Evaluation: From Precision, Recall and F-Measure to ROC, Informedness, Markedness \& Correlation"Journal of Machine Learning Technologies. 2(1): 37-63. Retrieved from: http://www.flinders.edu.au/science_engineering/fms/SchoolCSEM/publications/tech_reps-research_artfcts/TRRA_2007.pdf

Prieto, IM, \& Pilar Pérez Santana, M. (2012). Building ambidexterity: The role of human resource practices in the performance of firms from Spain. Human Resource Management, 51 (2), 189-211.

Pržulj, N. (2007). Biological comparison network using graphlet degree distribution. Bioinformatics, 23 (2), E177-E183.

$Q$

Quintane, E., \& Kleinbaum, AM (2011). Matter over mind? E-mail data and the measurement of social networks. Connections, 31 (1), 22-46

$\boldsymbol{R}$

Rabiner, L. R., \& Gold, B. (1975). Theory and application of digital signal processing. Englewood Cliffs, NJ, Prentice-Hall, Inc.

Raider, H., \& Krackhardt, D. J. (2001). Intraorganizational networks. In J. A. C. Baum (Ed.), Companion to organizations (pp. 58-74). Oxford, UK: Blackwell.

Reichertz, J. (2007). Abduction: The logic of discovery of grounded theory. The Sage handbook of grounded theory. Sage. 214-228. 
Ribeiro, M. T., Singh, S., \& Guestrin, C. (2016, August). Why should I trust you?: Explaining the predictions of any classifier. In Proceedings of the 22nd ACM SIGKDD international conference on knowledge discovery and data mining. 1135-1144

Richter, A., Dickmann, M., \& Graubner, M. (2008). Patterns of human resource management in consulting firms. Personnel Review, 37(2), 184-202.

Rico, R., Sánchez-Manzanares, M., Gil, F., \& Gibson, C. (2008). Team implicit coordination processes: A team knowledge-based approach. Academy of Management Review, 33(1), 163-184.

Rimé, B. \& Gaussin, J. (1982). Sensibilité des comportements non verbaux aux variations de la densité de communication. L'année psychologique, 82(1), 173-187.

Rimé, B. \& Schiaratura, L. (1991). Gesture and Speech. In Feldman, R. S., Feldman, R. S., \& Rimé, B. (Eds.). (1991). Fundamentals of nonverbal behavior. Cambridge University Press.

L., Hupet, M., \& Ghysselinckx, A. (1984). Effects of relative immobilization on the speaker's nonverbal behavior and on the dialogue imagery level. Motivation and Emotion, 8(4), 311-325.

Robinson, W.P. (1979). Speech markers in social class. In Scherer, K. R. and Giles, H. (Eds.). Social markers in speech. Cambridge University Press. 211-249

Rokach, L., \& Maimon, O. (2005). Top-down induction of decision trees classifiers-a survey. IEEE Transactions on Systems, Man, and Cybernetics, Part C (Applications and Reviews), 35(4), 476-487.

Rosing, K., Frese, M., \& Bausch, A. (2011). Explaining the heterogeneity of the leadershipinnovation relationship: Ambidextrous leadership. The Leadership Quarterly, 22(5), 956-974.

Roudposhti, K. K., Nunes, U., \& Dias, J. (2016). Probabilistic social behavior analysis by exploring body motion-based patterns. IEEE transactions on pattern analysis and machine intelligence, 38(8), 1679-1691.

Rozovsky, J. (2015). The five keys to a successful Google team. Retrieved from https://rework. withgoogle.com/blog/five-keys-to-a-successful-google-team/

Rubenstein-Montano, B., Liebowitz, J., Buchwalter, J., McCaw, D., Newman, B., Rebeck, K., \& Team, T. K. M. M. (2001). A systems thinking framework for knowledge management. Decision support systems, 31(1), 5-16.

Ruesch, J., Bateson, G., Pinsker, E. C., \& Combs, G. (2017). Communication: The social matrix of psychiatry. Routledge.

Ruscio, J., Whitney, D. M., \& Amabile, T. M. (1998). Looking inside the fishbowl of creativity: Verbal and behavioral predictors of creative performance. Creativity Research Journal, 11(3), 243-263.

Rust, R.T., Huang, M. H. (2014). The Service Revolution and the Transformation of Marketing Science. Marketing Science, 33(2):206-221.

$S$

Salas, E., Reyes, D. L., \& McDaniel, S. H. (2018). The science of teamwork: Progress, reflections, and the road ahead. American Psychologist, 73(4), 593. 
Shuffler, M. L., Thayer, A. L., Bedwell, W. L., \& Lazzara, E. H. (2015). Understanding and improving teamwork in organizations: A scientifically based practical guide. Human Resource Management, 54(4), 599-622.

Saldanha, T. J., Mithas, S., \& Krishnan, M. S. (2017). Leveraging Customer Involvement for Fueling Innovation: The Role of Relational and Analytical Information Processing Capabilities. MIS Quarterly, 41(1).

Santoro, J. M., Dixon, A. J., Chang, C. H., \& Kozlowski, S. W. (2015). Measuring and monitoring the dynamics of team cohesion: Methods, emerging tools, and advanced technologies. In Team cohesion: Advances in psychological theory, methods and practice (pp. 115-145). Emerald Group Publishing Limited.

Sawhney, M., Verona, G., \& Prandelli, E. (2005). Collaborating to create: The Internet as a platform for customer engagement in product innovation. Journal of Interactive Marketing, 19(4), 4-17.

Schacter, D. L. (1999). The seven sins of memory: Insights from psychology and cognitive neuroscience. American Psychologist, 54(3), 182.

Scherer, K. R. (1978). Personality inference from voice quality: The loud voice of extroversion. European Journal of Social Psychology, 8(4), 467-487.

(1979a). Speech and emotional states. In Darby, J. (Ed.). Speech evaluation in psychiatry. Grune \& Stratton. 189-220

(1979b). Voice and speech correlates of perceived social influence. In Giles, H. and St. Claire, R. (Eds.). The social psychology of language. Blackwell Publisher.

\& Scherer, U. (1981). Speech behavior and personality. In Darby, J. (Ed.). Speech evaluation in psychiatry. Grune \& Stratton. 115-135

(1989). Vocal correlates of emotion. In H. Wagner and A. Manstead (Eds.). Wagner, H. E., \& Manstead, A. E. Handbook of social psychophysiology. John Wiley \& Sons.

Schneider, B., Ehrhart, MG Mayer, DM, Saltz, JL, \& Niles-Jolly, K. (2005). Understanding organization-customer links in service settings. Academy of Management Journal, 48 (6), 1017-1032.

Schwartz, R. G., \& Teach, R. D. (2000). A model of opportunity recognition and exploitation: an empirical study of incubator firms. Journal of Research in Marketing and Entrepreneurship, 2(2), 93-107.

Schwarz, R. (2015) What the Research Tells Us About Team Creativity and Innovation. Harvard Business Review. Retrieved from https://hbr.org/2015/12/what-the-researchtells-us-about-team-creativity-and-innovation

Scissors, L. E., Gill, A. J., \& Gergle, D. (2008, November). Linguistic mimicry and trust in textbased CMC. In Proceedings of the 2008 ACM conference on Computer supported cooperative work (pp. 277-280). ACM.

Scott, J. (1988). Social network analysis. Sociology, 22(1), 109-127. (2017). Social network analysis. Sage.

Scott, K. A., Heathcote, J. M., \& Gruman, J. A. (2011). The diverse organization: Finding gold at the end of the rainbow. Human Resource Management, 5o(6), 735-755. 
Scott, S. G., \& Bruce, R. A. (1994). Determinants of innovative behavior: A path model of individual innovation in the workplace. Academy of Management Journal, 37(3), 580607.

Sethi, R., Smith, D. C., \& Park, C. W. (2001). Cross-functional product development teams, creativity, and the innovativeness of new consumer products. Journal of Marketing Research, 38(1), 73-85.

Shameer, K., Badgeley, M. A., Miotto, R., Glicksberg, B. S., Morgan, J. W., \& Dudley, J. T. (2016). Translational bioinformatics in the era of real-time biomedical, health care and wellness data streams. Briefings in bioinformatics, 118 .

Shannon, C. E., \& Weaver, W. (1963). The mathematical theory of communication. Urbana, IL: University of Illinois Press.

Shapiro, J. (2018). Help Your Team Understand What Data Is and Isn't Good For. Harvard Business Review. Retrieved from https://hbr.org/2018/10/help-your-teamunderstand-what-data-is-and-isnt-good-

for?utm_campaign=hbr\&utm_medium=social\&utm_source=twitter\&fbclid=IwAR16 MASwSwY-7KUZLTqTVCWixb2Lt7Rlu842Mnm5MmMFt_4-SqagWbRNgco

Shepard, C. A., Giles, H., \& Beth, A. (2001). Communication Accommodation Theory. In The New Handbook of Language and Social Psychology. W. Peter Robinson and Howard Giles (Eds). Chichester, Eng.: Wiley, 33-56.

Shmueli, E., Singh, UK, Lepri, B., \& Pentland, A. (2014). Sensing, understanding, and shaping social behavior. IEEE Transactions on Computational Social Systems, 1 (1), 22-34.

Simonton, D. K. (2003). Scientific creativity as constrained stochastic behavior: the integration of product, person, and process perspectives. Psychological Bulletin, 129(4), 475 .

Skålén, P., Gummerus, J., von Koskull, C., \& Magnusson, P. R. (2015). Exploring value propositions and service innovation: a service-dominant logic study. Journal of the Academy of Marketing Science, 43(2), 137-158.

Smith, P.M. (1980). Judging masculine and feminine social identities from content-controlled speech. In Clément, R., Giles, H., Robinson, W. P., \& Smith, P. M. (Eds.) Language: Social psychological perspectives. Pergamon. 121-126

Sociometric Solutions. (2013) Data Collection and Export recommendations for Researchers (2013) Sociometric Badge User Manual

Sommer, (1967). Sociofugal Space. American Journal of Sociology. The University of Chicago Press. 27(6): 654-66o.

Sonenshein, S. (2016). Routines and creativity: From dualism to duality. Organization Science, 27(3), 739-758.

Sosa, M. E. (2011). Where do creative interactions come from? The role of tie content and social networks. Organization Science, 22(1), 1-21.

Gargiulo, M., \& Rowles, C. (2015). Can informal communication networks disrupt coordination in new product development projects? Organization Science, 26 (4), 1059-1078.

Spanakis, G., Weiss, G., \& Roefs, A. (2016). Bagged Boosted Trees for Classification of Ecological Momentary Assessment Data. arXiv preprint arXiv:1607.01582.

Spence, M. (1973). Job market signaling. Quarterly Journal of Economics, 87, 355-374. 
Spence, M. (2002). Signaling in retrospect and the informational structure of markets. American Economic Review, 92(3), 434-459.

Statista. (2018). Retrieved from https://www.statista.com/statistics/629124/consultingmarket-size-europe/

Storey, C., \& Larbig, C. (2018). Absorbing customer knowledge: how customer involvement enables service design success. Journal of Service Research, 21(1), 101-118.

Strobl, C., Boulesteix, A. L., \& Augustin, T. (2007). Unbiased split selection for classification trees based on the Gini index. Computational Statistics E Data Analysis, 52(1), 483501.

Strübing, J. (2007). Research as pragmatic problem-solving: The pragmatist roots of empirically-grounded theorizing. In The Sage handbook of grounded theory. Sage. $580-602$.

Sue-Chan, C., \& Hempel, P. S. (2016). The Creativity-Performance Relationship: How Rewarding Creativity Moderates the Expression of Creativity. Human Resource Management, 55(4), 637-653.

Sun, R., Moore, E., \& Torres, J. F. (2009, April). Investigating glottal parameters for differentiating emotional categories with similar prosodics. In Acoustics, speech and signal processing, 2009. ICASSP 2009. IEEE international conference on (pp. 45094512). IEEE.

Sundbo, J. (1997). Management of innovation in services. Service Industries Journal, 17(3), 432-455.

Swan, M. 2013. “The Quantified Self: Fundamental Disruption in Big Data Science and Biological Discovery." Big Data, 1(2): 85-99.

Szulanski, G. (1996). Exploring internal stickiness: Impediments to the transfer of best practice within the firm. Strategic Management Journal, 17(S2), 27-43.

\section{T}

Teng, S. H. (2016). Scalable algorithms for data and network analysis. Foundations and Trends ${ }^{\circledR}$ in Theoretical Computer Science, $12(1-2), 1-274$.

Tether, B. S. (2005). Do services innovate (differently)? Insights from the European innobarometer survey. Industry \& Innovation, 12(2), 153-184.

Thamhain, H. J. (2003). Managing innovative R\&D teams. RED Management, 33(3), 297-311.

Timmermans, S., \& Tavory, I. (2012). Theory construction in qualitative research: From grounded theory to abductive analysis. Sociological Theory, 30(3), 167-186.

Toivonen, M., \& Tuominen, T. (2009). Emergence of innovations in services. The Service Industries Journal, 29(7), 887-902.

Trager, G. L. (1958). Paralanguage: A first approximation. Studies in Linguistics, 13, 1-12. (1960). Taos III: Paralanguage. Anthropological Linguistics, 2, 24-30.

(1961). The typology of paralanguage. Anthropological Linguistics, 3 (1), 17-21.

Traum, D. R., \& Heeman, P. A. (1996, August). Utterance units in spoken dialogue. In Workshop on Dialogue Processing in Spoken Language Systems (pp. 125-140). Springer, Berlin, Heidelberg.

Trevor, H., Robert, T., \& JH, F. (2009). The elements of statistical learning: data mining, inference, and prediction. Stanford, California. Retrieved from 
http://thuvien.thanglong.edu.vn:8081/dspace/bitstream/DHTL_1234567

89/4053/1/\%5BSpringer\%2oSeries\%20in\%2oStatistics-1.pdf

Tripathi, P., \& Burleson, W. (2011). Predicting creativity in the wild: experience sampling method and sociometric modeling of movement and face-to-face interactions in teams. Arizona State University.

Trischler, J., Pervan, S. J., Kelly, S. J., \& Scott, D. R. (2018). The value of codesign: the effect of customer involvement in service design teams. Journal of Service Research, 21(1), 75100.

$\boldsymbol{V}$

van de Ven, A. H. (1986). Central problems in the management of innovation. Management Science, 32(5), 590-607.

van Woerkom, M., \& Meyers, M. C. (2015). My Strengths Count! Human Resource Management, 54(1), 81-103.

Verganti, R. (2008). Design, meanings, and radical innovation: A metamodel and a research agenda. Journal of Product Innovation Management, 25(5), 436-456.

Villarejo, M. V., Zapirain, B. G., \& Zorrilla, A. M. (2012). A stress sensor based on Galvanic Skin Response (GSR) controlled by ZigBee. Sensors, 12(5), 6075-6101.

Vinciarelli, A., Pantic, M., \& Bourlard, H. (2009). Social signal processing: Survey of an emerging domain. Image and Vision Computing, 27(12), 1743-1759.

Von Hippel, E. (1994). "Sticky information" and the locus of problem solving: implications for innovation. Management Science, 40(4), 429-439.

$\boldsymbol{W}$

Waber, B. N., Olguín-Olguín, D., Kim, T., Mohan, A., Ara, K., \& Pentland, A. (2007). Organizational engineering using sociometric badges. Retrieved from http://web.media.mit.edu/ bwaber/Waber-NetSci-o7.pdf

Wallbott, H. G., \& Scherer, K. R. (1986). Cues and channels in emotion recognition. Journal of Personality and Social Psychology, 51(4), 690.

WEKA (2017a). WEKA Random Forest Source. Retrieved from:http://weka.sourceforge.net/doc.dev/index. html?weka/classifiers/trees/ REPTree.html

(2017b). WEKA Multiclass Classifier Source. Retrieved from: http://weka.sourceforge.net/doc .dev/weka/classifiers/meta/MultiClassClassifier. html Backfitting Retrieved from: http://weka. sourceforge.net/doc.dev/weka/classifiers/trees/RandomForest.html

Wiemann, J. M., \& Knapp, M. L. (1975). Turn-taking in conversations. Journal of communication, 25(2), 75-92.

Wilson, T. D., \& Schooler, J. W. (1991). Thinking too much: introspection can reduce the quality of preferences and decisions. Journal of Personality and Social Psychology, 6o(2), 181. 
Witell, L., Snyder, H., Gustafsson, A., Fombelle, P., \& Kristensson, P. (2016). Defining service innovation: A review and synthesis. Journal of Business Research, 69(8), 2863-2872.

Wood, F. S., \& Gorman, J. W. (1971). Fitting equations to data: computer analysis of multifactor data for scientists and engineers. Wiley-Interscience.

Woolley, A. W., Chabris, C. F., Pentland, A., Hashmi, N., \& Malone, T. W. (2010). Evidence for a collective intelligence factor in the performance of human groups. Science, 330(6004), 686-688.

\section{$Y$}

Yang, Y., Chawla, NV, Walter Licht, RN, \& Dong, Y. (2016). Influence Activation Model: A New Perspective in Social Influence Analysis and Social Network Evolution. arXiv preprint arXiv: 1605.08410.

Yu, D., Blocker, R. C., Sir, M. Y., Hallbeck, M. S., Hellmich, T. R., Cohen, T., ... \& Pasupathy, K. S. (2016). Intelligent Emergency Department: Validation of Sociometers to Study Workload. Journal of Medical Systems, 40(3), 1-12.

\section{$Z$}

Zagenczyk, T. J., Purvis, R. L., Shoss, M. K., Scott, K. L., \& Cruz, K. S. (2015). Social influence and leader perceptions: Multiplex social network ties and similarity in leadermember exchange. Journal of Business and Psychology, 30(1), 105-117.

Zhou, L., Pan, S., Wang, J., \& Vasilakos, A. V. (2017). Machine learning on big data: Opportunities and challenges. Neurocomputing, 237, 350-361.

Zhou, Y., Hong, Y., \& Liu, J. (2013). Internal commitment or external collaboration? The impact of human resource management systems on firm innovation and performance. Human Resource Management, 52(2), 263-288.

Zippo, A. G., Storchi, R., Nencini, S., Caramenti, G. C., Valente, M., \& Biella, G. E. M. (2013). Neuronal functional connection graphs among multiple areas of the rat somatosensory system during spontaneous and evoked activities. PLoS Computational Biology, 9(6), e1003104. 


\section{Summary}


This dissertation emphasized the importance of quantifying the objective behavior of teams and aimed to find how team creative problem solving can be explained by objective and finegrained behaviors in real-life new service development (NSD) processes. This thesis investigated the application of wearable technology for the study of objective human behavioral cues or "social signals" such as vocalics, proxemics, and kinesics to generate insights into the dynamics of NSD teams.

Chapter 2 introduced the potential of social signals as new types of data for social science research collected with wearable technologies. We presented the sociometric badge as a viable tool for detecting and recording a wide range of social signals. However, as most variables collected have insufficient theoretical foundations for constructing hypotheses we propose employing machine learning algorithms "less-traditional approach" (for social sciences) to analyzing data. To alleviate the lack of theoretical framing for hypotheses development, we discuss data-driven theory development as a viable alternative for traditional theory building approaches. The chapter concluded with a step-by-step best practice procedure of conducting a study with wearable devices.

Chapter 3 focused on proximity and information flow as a point of departure for investigating actual face-to-face encounters between actors. In this empirical study we objectively measured communication patterns as factors shaping creative outcomes in new service development (NSD) teams. This chapter presented preliminary evidence linking proxemics and communicative measures of 'honest social signals' as markers of creative success within teams. The multiple regressions with quadratic assignment procedure was compared with multi-class classification revealing the roles of different objective measures gathered using wearable computing devices. The practical implications of this chapter outline the importance of nonverbal behavior in affecting as well as predicting creativity in the ideation phase of the process, proving that creativity is the outcome of complex social interactions. The main implication for service research is that the converging stage of the ideation process the structure of the project meeting itself is highly important.

The premise of Chapter 4 was that some social relationships (i.e., ties) are more important for the creative process than others during the new service development process. We introduce the concept of "creative activation" defined as the convergence between creative ties and social signals. The main contribution of the paper is successfully applying dynamic network alignment in social science (previously solely used in bio-informatics) to examine the real-time interactions (i.e., social signals) generated by two new service development (NSD) teams, and their participating customers during actual company meetings. This formalized comparison of networks rendered (a) network topological evidence of creative activation in teams, and, (b) isolate patterns in social signals (i.e. interaction synchrony) at different points in time that contribute most to this result. 
About the author 
R. Elena Francu was born in Pitești, Romania, on October $2^{\text {nd }} 1987$. She completed her High school diploma in 2006 at Ion C. Bratianu College with a Mathematics and Computer Science specialization. She then went on to complete a BSc. in Political Science with a Sociology specialization in 2009 and a MSc. in Sociology with scholarship in 2011 at S,coala Națională de Studii Politice și Administrative (SNSPA), Bucharest. From 2010 to 2012, she held a range of positions working in academic research and teaching administration.

Following this, Elena first joined Maastricht University in 2012, working as a Deputy Project Leader at the Service Science Factory. In 2013, she began her Ph.D. candidacy under the supervision of Prof. Dr. Jos Lemmink, Prof. Dr. Mien Segers, and Dr. Piet van den Bossche. Her current line of computational social science research involved working on a number of industry case studies in sectors such as software and ICT, financial services, medical technology, professional services, veterinary pharmaceutics, and education.

During this time, she also taught a number of BSc. courses at Maastricht University, and supervised numerous post-graduate research dissertation projects. Her other teaching accomplishments include the development of a ubiquitous computing and social network analysis workshop for MSc students, and roles as a Maastricht University SBE Premium Honors Program coach and mentor.

Her research has accepted at leading international doctoral symposia such as the European Marketing Academy (EMAC) Doctoral Colloquium, May 2015, held in Leuven, Belgium and AMA SERVSIG (Services Special Interest Group of the American Marketing Association) Doctoral Consortium, June 2015, held in San Jose, CA, U.S.A. Elena's working papers have also appeared in the proceedings of leading international conferences such as the SERVSIG Conference June 2016, held in Maastricht, The Netherlands, and the Frontiers in Service Conference, June 2016, held in Bergen, Norway.

Elena has given invited research talks at other leading European institutions such as the University of Cambridge and the University of Vienna. Furthermore, Elena has served several times as a reviewer for the Industrial Marketing Management journal. 
